\title{
Higher-Order Bulk-Boundary Correspondence for Topological Crystalline Phases
}

\author{
Luka Trifunovic and Piet W. Brouwer \\ Dahlem Center for Complex Quantum Systems and Physics Department, Freie Universität Berlin, \\ Arnimallee 14, 14195 Berlin, Germany
}

(Received 7 May 2018; published 22 January 2019)

\begin{abstract}
We study the bulk-boundary correspondence for topological crystalline phases, where the crystalline symmetry is an order-two (anti)symmetry, unitary or antiunitary. We obtain a formulation of the bulkboundary correspondence in terms of a subgroup sequence of the bulk classifying groups, which uniquely determines the topological classification of the boundary states. This formulation naturally includes higherorder topological phases as well as topologically nontrivial bulk systems without topologically protected boundary states. The complete bulk and boundary classification of higher-order topological phases with an additional order-two symmetry or antisymmetry is contained in this work.
\end{abstract}

DOI: 10.1103/PhysRevX.9.011012

Subject Areas: Condensed Matter Physics

\section{INTRODUCTION}

A central paradigm in the field of topological insulators and superconductors is the bulk-boundary correspondence: A nontrivial topology of the bulk band structure uniquely manifests itself through an anomalous gapless, topologically nontrivial boundary, irrespective of the orientation of the boundary or the lattice termination [1-3]. On the other hand, for topological crystalline phases, which are protected by an additional nonlocal crystalline symmetry [4-26], the existence of gapless boundary states for a nontrivial bulk topology is guaranteed only if the boundary is invariant under the crystalline symmetry.

Recently, it was realized that a nontrivial crystalline topology of a $d$-dimensional crystal may also manifest itself through protected boundary states of dimension less than $d-1$ [27-40]. A topological phase with such lowerdimensional boundary states is called a "higher-order topological phase," where the order $n$ of the topological phase corresponds to the codimension of the boundary states [28]. [According to this definition, a topological insulator or superconductor with the conventional $(d-1)$-dimensional boundary states is a first-order topological phase.] The condition that guarantees the protection of such higherorder boundary states is that the orientation of the crystal faces and the lattice termination be compatible with the crystalline symmetry-i.e., the crystal faces and the corresponding lattice termination must be related to each other by the crystalline symmetry operation. This is a much weaker

Published by the American Physical Society under the terms of the Creative Commons Attribution 4.0 International license. Further distribution of this work must maintain attribution to the author(s) and the published article's title, journal citation, and DOI. condition than the condition that the crystal boundary be invariant under the symmetry operation (compare with Fig. 1). For example, whereas inversion symmetry leaves no crystal faces invariant, compatibility with inversion symmetry merely requires that crystal faces appear in inversion-related pairs [see Fig. 1(c)]. Topological crystalline insulators with second-order boundary states were theoretically predicted for models with certain magnetic symmetries [28], mirror symmetry [28,30], and rotation and inversion symmetries [20,29,31,41-43]. The latter two symmetries are relevant for the semimetal $\mathrm{Bi}$, which shows boundary states reminiscent of that of a second-order topological insulator [44].

The presence of a crystalline symmetry is not a necessary requirement for the boundary phenomenology associated with a higher-order phase. Indeed, early examples of protected codimension-two boundary states include the superfluid ${ }^{3} \mathrm{He}-\mathrm{B}$ phase [45] and a three-dimensional topological insulator with a suitable time-reversal-breaking perturbation $[46,47]$, neither of which rely on the protection

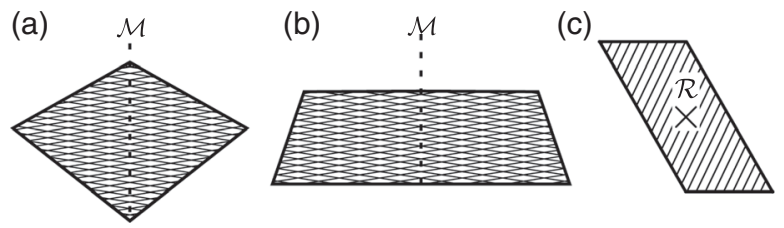

FIG. 1. Schematic pictures of a two-dimensional crystal for which the shape is compatible with mirror symmetry [(a) and (b)] and with twofold rotation symmetry (c). The crystal in (b) has a boundary that is invariant under the mirror symmetry, whereas the boundaries of the crystals in (a) and (c) appear in symmetryrelated pairs. The special situation of a crystal with mirrorsymmetric boundary, as shown in panel (b), is excluded from the definition of the higher-order topological phases. 
by a bulk crystalline symmetry. Instead, in these cases the appearance of higher-order protected boundary states can be solely attributed to a boundary termination that is itself topologically nontrivial, whereas the underlying bulk is essentially trivial. In Ref. [41] we called these termination-dependent higher-order topological phases extrinsic, to contrast them with the anomalous (intrinsic), termination-independent higher-order boundary states of topological crystalline phases. Although for anomalous higher-order topological phases, too, the precise form of the $(d-2)$-dimensional boundary states may still depend on details of the lattice termination, their very existence is a consequence of a nontrivial bulk topology and is protected as long as the crystal termination remains compatible with the crystalline symmetry.

While a complete classification of higher-order topological phases (HOTPs) is still lacking, several authors have obtained partial classifications of higher-order topological phases, restricted to certain crystalline symmetries or for a certain tenfold-way class $[20,30,41,48]$. (The tenfold-way or Altland-Zirnbauer classes are defined with respect to the presence or absence of the fundamental nonspatial symmetry operations time-reversal $\mathcal{T}$, particle-hole conjugation $\mathcal{P}$ and the chiral operation $\mathcal{C}=\mathcal{P} \mathcal{T}$ [49]). Two approaches have been taken for the classification of anomalous, termination-independent HOTPs: A bulk-based approach, which starts from the classification of the bulk band structure and then shows under which circumstances a nontrivial bulk topology implies a higher-order topological phase $[30,41]$, and a boundary-based approach, in which all topologically nontrivial boundaries of HOTPs are classified first, and a classification of anomalous, terminationindependent HOTPs is obtained upon identification of boundary states that are related by a change of termination $[20,41,48]$. For crystalline phases with an order-two crystalline symmetry, for which a complete classification of the bulk topology exists [21], the two approaches were found to be in complete agreement for the second-order topological phases [41,48]. The boundary-based approach not only classifies the anomalous, termination-independent HOTPs, but also the extrinsic higher-order topological phases, for which the higher-order boundary states are a manifestation of a nontrivial boundary topology rather than a nontrivial bulk topology.

In this work, we provide a full classification of higherorder topological phases with an order-two crystalline symmetry or antisymmetry, for arbitrary order $n$ of the topological phase and in arbitrary spatial dimension $d$. A crystalline symmetry or antisymmetry $\mathcal{S}$ is called "ordertwo" if $\mathcal{S}^{2}= \pm 1$. Its spatial type is determined by the number $d_{\|}$of inverted dimensions, such that $d_{\|}=0$ correspond to on-site (anti)symmetry, and $d_{\|}=1,2,3$ to mirror, twofold rotation, and inversion (anti)symmetry, respectively.

We present classifications both from a bulk perspective and from a boundary perspective. Our bulk classification of
HOTPs with an order-two crystalline symmetry refines the existing classification of Shiozaki and Sato [21], who classified topological crystalline phases without accounting for the type of the boundary signatures. Whereas Ref. [21] described the topological classification in terms of a single classifying group $K$, our refined classification takes the form of a subgroup series

$$
K^{(d)} \subseteq \cdots \subseteq K^{\prime \prime} \subseteq K^{\prime} \subseteq K,
$$

which resolves the topological crystalline phases according to their associated anomalous boundary signature. The last term in Eq. (1) $K \equiv K^{(0)}$ is the classifying group of Ref. [21], which classifies the bulk band structure with an order-two symmetry or antisymmetry. The other terms $K^{(n)} \subseteq K$ are subgroups that exclude topological phases that are of order $n$ and lower for any crystal shape consistent with the crystalline symmetry. An illustration of the definitions of the groups $K^{(n)}$ is shown in Fig. 2 for the case of a three-dimensional crystal with twofold rotation symmetry. The subgroup $K^{\prime}$, which classifies topological crystalline phases that are not first order was previously studied in Ref. [41] in the context of crystals with mirror, twofold rotation, or inversion symmetry, where it was called the "purely crystalline subgroup." Note that the definition of the groups $K^{(n)}$ excludes crystals with boundary states that can be removed by a symmetryrespecting deformation of the crystal, such as the gapless surface states on a mirror-symmetric surface of a mirrorsymmetric crystal, compare Figs. 1(a) and 1(b).

The bulk classification is complemented with a classification of anomalous boundary states of codimension $n$, described by the boundary classification group $\mathcal{K}_{a}^{(n)}$. We show that there exists a "bulk-boundary correspondence," a general relation between the boundary classification group

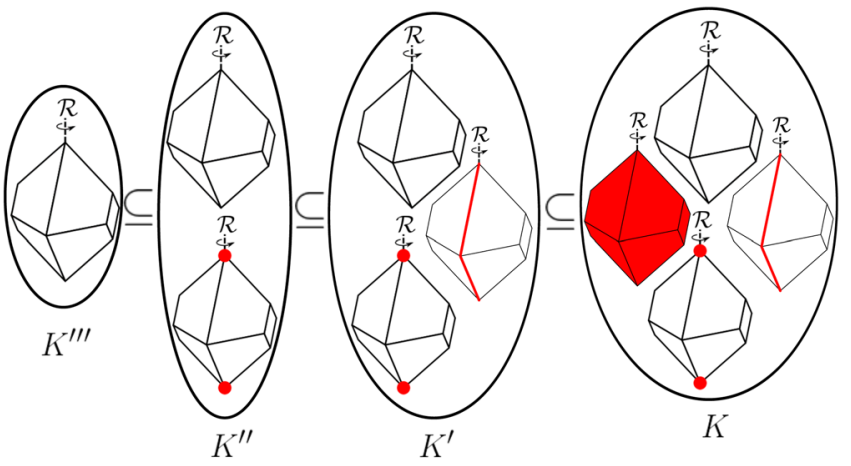

FIG. 2. The bulk classifying group $K$ classifies all bulk phases, regardless of the existence or type of anomalous boundary states. Refined classification groups $K^{(n)}$ are obtained by excluding topological phases with anomalous boundary states of codimensions $\leq n$. The figure illustrates this procedure for a crystal with a twofold rotation symmetry $\mathcal{R}$. Anomalous boundary states are indicated in red. 
$\mathcal{K}_{a}^{(n)}$ and the subgroup series (1) of the bulk classification groups,

$$
\mathcal{K}_{a}^{(n+1)}=K^{(n)} / K^{(n+1)}, \quad n=0,1,2, \ldots, d .
$$

In case the number of inverted dimensions $d_{\|}<d$ the subgroup series (1) starts with one or more trivial groups,

$$
K^{(n)}=0 \quad \text { for } n>d_{\|},
$$

so that Eq. (2) yields a complete bulk-boundary correspondence for order-two crystalline symmetries: A topologically nontrivial bulk is uniquely associated with a higher-order topological phase. On the other hand, if $d_{\|}=$ $d$ (inversion symmetry), the first group in the subgroup series (1) $K^{(d)}$ may be nontrivial. In that case there is only a partial bulk-boundary correspondence and $K^{(d)}$ classifies the topological crystalline phases without topologically protected boundary states, see Fig. 2. Topological phases contained in $K^{(d)}$ are smoothly connected to atomic-limit insulators or superconductors. A nontrivial classifying group $K^{(d)}$ indicates the existence of multiple topologically distinct atomic-limit phases [22,50,51].

All of the above results will be expanded and made more precise in Sec. IV. There, we present a fully algebraic proof of the central relation (2), making essential use of an "order-raising" homomorphism $\omega$, which maps the classifying group $K^{(n)}$ for a $d$-dimensional crystal onto the group $K^{(n+1)}$ of a $(d+1)$-dimensional crystal, while keeping the spatial and nonspatial symmetries the same, except for an increase of the number of inverted dimensions $d_{\|}$by one. We construct the homomorphism $\omega$ by combination of two maps known from the literature: The "dimension-raising isomorphisms," which relate bulk classifying groups $K \equiv$ $K^{(0)}$ in different dimensions and different tenfold-way symmetry classes; see Refs. [21,52], and the dimensionlowering "boundary map" of Ref. [6]. The map $\omega$ represents the same homomorphism between classifying groups as the "stacking construction" of Refs. [53-55], which was used recently for the construction of higher-order topological phases out of lower-dimensional structures $[20,48]$. It is the algebraic formulation in terms of the dimensionraising isomorphism and the boundary map that allows us to obtain the bulk-boundary correspondence (2) by purely algebraic methods.

The remainder of this article is organized as follows: In Sec. II we review the classification of the topological crystalline phase stabilized by an order-two symmetry, and introduce the dimension-raising isomorphisms, closely following previous work by Shiozaki and Sato [21]. In Sec. III we discuss Hamiltonians of "canonical form" and show how higher-order phases naturally arise from the presence of crystalline-symmetry-breaking mass terms, generalizing the conclusions of Refs. [28,30,41] for second-order topological phases. In Secs. IV and V we give the formal definitions of the classifying groups $K^{(n)}$ and $\mathcal{K}_{a}^{(n)}$, construct the order-raising homomorphism $\omega$, obtain explicit expressions, and establish the bulkboundary correspondence (2) using algebraic methods. Section VI discusses a few representative examples of this general classification and shows how the homomorphism $\omega$ relates classification results in different dimensions to each other. In Sec. VII we construct a procedure for lowering the dimension $d$ of the crystal, while preserving the dimension of the anomalous boundary states, providing a general realization of an idea put forward by Matsugatani and Watanabe [56]. Finally, turning the arguments of our article around, the bulk-boundary correspondence (2) can be used to obtain the bulk classifying groups from the classification of anomalous boundaries together with the classification of the topologically nontrivial atomic limits, thus providing a boundary-based alternative to the $K$-theory-based classification of Ref. [21]. This program is carried out in Sec. VIII. We conclude in Sec. IX. The Appendixes contain derivations not presented in the main text as well as a few additional results.

\section{SHIOZAKI-SATO CLASSES FOR TOPOLOGICAL PHASES WITH AN ORDER-TWO SYMMETRY}

The tenfold-way or Altland-Zirnbauer [49] classes are defined according to the presence or absence of timereversal symmetry $\mathcal{T}$, particle-hole antisymmetry $\mathcal{P}$, and chiral antisymmetry $\mathcal{C}$; see Table I. Shiozaki and Sato [21] extend the tenfold-way classes to include an additional crystalline unitary symmetry $[8,9,16]$, unitary antisymmetry, antiunitary symmetry, or antiunitary antisymmetry $\mathcal{S}$. The crystalline symmetry is an order-two symmetry, which means that its square is proportional to the identity operation.

TABLE I. The tenfold-way classes are defined according to the presence or absence of time-reversal symmetry $(\mathcal{T})$, particle-hole antisymmetry $(\mathcal{P})$, and chiral antisymmetry $(\mathcal{C})$. The entries $\mathcal{T}^{ \pm}$ $\left(\mathcal{P}^{ \pm}\right)$denote that $\mathcal{T}^{2}= \pm 1\left(\mathcal{P}^{2}= \pm 1\right)$. The chiral antisymmetry is assumed to square to one.

\begin{tabular}{lllll}
\hline \hline Cartan & $s$ & $\mathcal{T}$ & $\mathcal{P}$ & $\mathcal{C}$ \\
\hline A & 0 & $\ldots$ & $\ldots$ & $\ldots$ \\
AIII & 1 & $\cdots$ & $\cdots$ & $\mathcal{C}$ \\
\hline AI & 0 & $\mathcal{T}^{+}$ & $\ldots$ & $\ldots$ \\
BDI & 1 & $\mathcal{T}^{+}$ & $\mathcal{P}^{+}$ & $\mathcal{C}$ \\
D & 2 & $\ldots$ & $\mathcal{P}^{+}$ & $\ldots$ \\
DIII & 3 & $\mathcal{T}^{-}$ & $\mathcal{P}^{+}$ & $\mathcal{C}$ \\
AII & 4 & $\mathcal{T}^{-}$ & $\ldots$ & $\ldots$ \\
CII & 5 & $\mathcal{T}^{-}$ & $\mathcal{P}^{-}$ & $\mathcal{C}$ \\
C & 6 & $\ldots$ & $\mathcal{P}^{-}$ & $\ldots$ \\
CI & 7 & $\mathcal{T}^{+}$ & $\mathcal{P}^{-}$ & $\mathcal{C}$ \\
\hline \hline
\end{tabular}


TABLE II. Bulk classification sequence $K^{(d)} \subseteq \cdots \subseteq K^{\prime} \subseteq K$ for zero- $(d=0)$, one- $(d=1)$, and twodimensional $(d=2)$ typological crystalline insulators and superconductors with an order-two crystalline symmetry or antisymmetry for the complex tenfold-way classes. The symbols $\mathcal{O}, \mathcal{M}$, and $\mathcal{R}$ refer to a local on-site $\left(d_{\|}=0\right)$, mirror $\left(d_{\|}=1\right)$, and twofold rotation symmetry $\left(d_{\|}=2\right)$, respectively.

\begin{tabular}{|c|c|c|c|c|c|c|c|c|}
\hline & & & $d=0$ & $d=1$ & $d=1$ & $d=2$ & $d=2$ & $d=2$ \\
\hline Class & $s$ & $t$ & $\mathcal{O}$ & $\mathcal{O}$ & $\mathcal{M}$ & $\mathcal{O}$ & $\mathcal{M}$ & $\mathcal{R}$ \\
\hline $\mathrm{A}^{\mathcal{S}}$ & 0 & 0 & $\mathbb{Z}^{2}$ & $0 \subseteq 0$ & $\mathbb{Z} \subseteq \mathbb{Z}$ & $0 \subseteq 0 \subseteq \mathbb{Z}^{2}$ & $0 \subseteq 0 \subseteq 0$ & $\mathbb{Z} \subseteq \mathbb{Z} \subseteq \mathbb{Z}^{2}$ \\
\hline $\mathrm{AIII}^{\mathcal{S}_{+}}$ & 1 & 0 & 0 & $0 \subseteq \mathbb{Z}^{2}$ & $0 \subseteq 0$ & $0 \subseteq 0 \subseteq 0$ & $0 \subseteq \mathbb{Z} \subseteq \mathbb{Z}$ & $0 \subseteq 0 \subseteq 0$ \\
\hline $\mathrm{A}^{C S}$ & 0 & 1 & 0 & $0 \subseteq \mathbb{Z}$ & $0 \subseteq 0$ & $0 \subseteq 0 \subseteq 0$ & $0 \subseteq \mathbb{Z} \subseteq \mathbb{Z}^{2}$ & $0 \subseteq 0 \subseteq 0$ \\
\hline $\mathrm{AIII}^{\mathcal{S}_{-}}$ & 1 & 1 & $\mathbb{Z}$ & $0 \subseteq 0$ & $\mathbb{Z} \subseteq \mathbb{Z}^{2}$ & $0 \subseteq 0 \subseteq \mathbb{Z}$ & $0 \subseteq 0 \subseteq 0$ & $2 \mathbb{Z} \subseteq \mathbb{Z} \subseteq \mathbb{Z}$ \\
\hline
\end{tabular}

It is sufficient to distinguish symmetry operations that square to one (labeled by $\eta_{\mathcal{S}}=+$ ) and to minus one $\left(\eta_{\mathcal{S}}=-\right)$. Further, the algebraic structure of the crystalline symmetry is characterized by signs $\eta_{\mathcal{T}, \mathcal{P}, \mathcal{C}}$ indicating whether $\mathcal{S}$ commutes $(\eta=+)$ or anticommutes $(\eta=-)$ with the time-reversal operation $\mathcal{T}$, particle-hole conjugation $\mathcal{P}$, or the chiral symmetry operation $\mathcal{C}$. Following Ref. [21], we denote the number of spatial degrees of freedom (d.o.f.) that are inverted under the crystalline symmetry operation by $d_{\|}$, so that on-site symmetries $\mathcal{O}$ have $d_{\|}=0$, reflections $\mathcal{M}$ have $d_{\|}=1$, twofold rotations $\mathcal{R}$ have $d_{\|}=2$, and inversion $\mathcal{I}$ has $d_{\|}=3$. Specifically, unitary symmetry $\left(\sigma_{\mathcal{S}}=1\right)$ and antisymmetry $\left(\sigma_{\mathcal{S}}=-1\right)$ operations are represented by unitary matrices $U_{\mathcal{S}}$,

$$
H(\boldsymbol{k}, m)=\mathcal{S} H(\boldsymbol{k}, m) \equiv \sigma_{\mathcal{S}} U_{\mathcal{S}} H(\mathcal{S} \boldsymbol{k}, m) U_{\mathcal{S}}^{-1},
$$

with $\mathcal{S} \boldsymbol{k}=\left(-\boldsymbol{k}_{\|}, \boldsymbol{k}_{\perp}\right), \boldsymbol{k}_{\|}=\left(k_{1}, \ldots, k_{d_{\|}}\right), \boldsymbol{k}_{\perp}=\left(k_{d_{\|}+1}, \ldots, k_{d}\right)$ and $U_{\mathcal{S}}^{2}=\eta_{\mathcal{S}}, U_{\mathcal{S}} U_{\mathcal{T}}=\eta_{\mathcal{T}} U_{\mathcal{T}} U_{\mathcal{S}}^{*}, U_{\mathcal{S}} U_{\mathcal{P}}=\eta_{\mathcal{P}} U_{\mathcal{P}} U_{\mathcal{S}}^{*}$, and $U_{\mathcal{S}} U_{\mathcal{C}}=\eta_{\mathcal{C}} U_{\mathcal{C}} U_{\mathcal{S}}$. Similarly, antiunitary symmetry and antisymmetry operations are represented as

$$
H(\boldsymbol{k}, m)=\mathcal{S} H(\boldsymbol{k}, m) \equiv \sigma_{\mathcal{S}} U_{\mathcal{S}} H^{*}(-\mathcal{S} \boldsymbol{k}, m) U_{\mathcal{S}}^{-1},
$$

such that $U_{\mathcal{S}} U_{\mathcal{S}}^{*}=\eta_{\mathcal{S}}, \quad U_{\mathcal{S}} U_{\mathcal{T}}^{*}=\eta_{\mathcal{T}} U_{\mathcal{T}} U_{\mathcal{S}}^{*}, \quad U_{\mathcal{S}} U_{\mathcal{P}}^{*}=$ $\eta_{\mathcal{P}} U_{\mathcal{P}} U_{\mathcal{S}}^{*}$, and $U_{\mathcal{S}} U_{\mathcal{C}}^{*}=\eta_{\mathcal{C}} U_{\mathcal{C}} U_{\mathcal{S}}$.
The above characterization of unitary and antiunitary symmetry operations by the signs $\eta_{\mathcal{S}, \mathcal{T}, \mathcal{P}, \mathcal{C}}$ and $\sigma_{\mathcal{S}}$ may be redundant [21], because symmetry operations that are characterized differently may be mapped onto each other. For example, if $H$ satisfies a crystalline unitary symmetry operation $\mathcal{S}$ which squares to one, then it also satisfies the unitary symmetry operation $i \mathcal{S}$, which squares to minus one, or (provided $\mathcal{T}$ symmetry is present) it satisfies the antiunitary symmetry $\mathcal{T S}$. Using such equivalences, Shiozaki and Sato group the symmetry operations $\mathcal{S}$ into "equivalence classes," which, together with the tenfold-way class of Table I, are labeled by one integer $s$ or by two integers $s$ and $t$. In this work, (as in Ref. [41]) we label the equivalence classes by representative (anti)symmetries that consist of a unitary crystalline symmetry $\mathcal{S}$ squaring to one or the product of such a crystalline symmetry and $\mathcal{T}, \mathcal{P}$, or $\mathcal{C}$. These representatives are summarized in the first column of Tables II-IV for the complex tenfold-way classes with unitary (anti)symmetries, the complex tenfold-way classes with antiunitary (anti)symmetries, and the real tenfold-way classes with unitary (anti)symmetries, respectively. For the complex tenfold-way classes with antiunitary (anti)symmetries we implicitly assume that $\mathcal{T}, \mathcal{P}$ commute with $\mathcal{S}$ when constructing these representatives; see Table VI.

The classification of topological phases (with or without the additional crystalline symmetry or antisymmetry) has a group structure, and the symbol $K($ or $\mathcal{K})$ is used to denote

TABLE III. Same as Table II, but for antiunitary symmetries and antisymmetries.

\begin{tabular}{lccccccc}
\hline \hline & & $d=0$ & $d=1$ & $d=1$ & $d=2$ & $d=2$ & $d=2$ \\
\cline { 2 - 7 } Class & $s$ & $\mathcal{O}$ & $\mathcal{O}$ & $\mathcal{M}$ & $\mathcal{O}$ & $\mathcal{M}$ & $\mathcal{R}$ \\
\hline $\mathrm{A}^{\mathcal{T}^{+} \mathcal{S}}$ & 0 & $\mathbb{Z}$ & $0 \subseteq 0$ & $\mathbb{Z}_{2} \subseteq \mathbb{Z}_{2}$ & $0 \subseteq 0 \subseteq 0$ & $0 \subseteq 0 \subseteq \mathbb{Z}$ & $\mathbb{Z}_{2} \subseteq \mathbb{Z}_{2} \subseteq \mathbb{Z}_{2}$ \\
$\mathrm{AIIII}^{\mathcal{P}^{+} \mathcal{S}_{+}}$ & 1 & $\mathbb{Z}_{2}$ & $0 \subseteq \mathbb{Z}$ & $\mathbb{Z}_{2} \subseteq \mathbb{Z}_{2}$ & $0 \subseteq 0 \subseteq 0$ & $0 \subseteq \mathbb{Z}_{2} \subseteq \mathbb{Z}_{2}$ & $0 \subseteq 0 \subseteq 0$ \\
$\mathrm{~A}^{\mathcal{P}^{+} \mathcal{S}}$ & 2 & $\mathbb{Z}_{2}$ & $0 \subseteq \mathbb{Z}_{2}$ & $0 \subseteq 0$ & $0 \subseteq 0 \subseteq \mathbb{Z}$ & $0 \subseteq \mathbb{Z}_{2} \subseteq \mathbb{Z}_{2}$ & $0 \subseteq 0 \subseteq 2 \mathbb{Z}$ \\
$\mathrm{AIIII}^{\mathcal{T}^{-} \mathcal{S}_{-}}$ & 3 & 0 & $0 \subseteq \mathbb{Z}_{2}$ & $0 \subseteq 2 \mathbb{Z}$ & $0 \subseteq 0 \subseteq \mathbb{Z}$ & $0 \subseteq 0 \subseteq 0$ & $0 \subseteq 0 \subseteq 0$ \\
$\mathrm{~A}^{\mathcal{T}^{-} \mathcal{S}}$ & 4 & $2 \mathbb{Z}$ & $0 \subseteq 0$ & $0 \subseteq 0$ & $0 \subseteq 0 \subseteq \mathbb{Z}_{2}$ & $0 \subseteq 0 \subseteq 2 \mathbb{Z}$ & $0 \subseteq 0 \subseteq 0$ \\
$\mathrm{AIIII}^{\mathcal{P}^{-} \mathcal{S}_{+}}$ & 5 & 0 & $0 \subseteq 2 \mathbb{Z}$ & $0 \subseteq 0$ & $0 \subseteq 0 \subseteq 0$ & $0 \subseteq 0 \subseteq 0$ & $0 \subseteq 0 \subseteq 0$ \\
$\mathrm{~A}^{\mathcal{P}^{-} \mathcal{S}}$ & 6 & 0 & $0 \subseteq 0$ & $0 \subseteq 0$ & $0 \subseteq 0 \subseteq 2 \mathbb{Z}$ & $0 \subseteq 0 \subseteq 0$ & $0 \subseteq 0 \subseteq \mathbb{Z}$ \\
$\mathrm{AIIII}^{\mathcal{T}^{+} \mathcal{S}_{-}}$ & 7 & 0 & $0 \subseteq 0$ & $0 \subseteq \mathbb{Z}$ & $0 \subseteq 0 \subseteq 0$ & $0 \subseteq 0 \subseteq 0$ & $0 \subseteq \mathbb{Z}_{2} \subseteq \mathbb{Z}_{2}$ \\
\hline \hline
\end{tabular}


TABLE IV. Bulk classification sequence $K^{(d)} \subseteq \cdots \subseteq K^{\prime} \subseteq K$ for zero- $(d=0)$, one- $(d=1)$, and two-dimensional $(d=2)$ topological crystalline phases with an order-two crystalline symmetry or antisymmetry for the real tenfold-way classes. The symbols $\mathcal{O}$, $\mathcal{M}$, and $\mathcal{R}$ refer to a local on-site $\left(d_{\|}=0\right)$, mirror $\left(d_{\|}=1\right)$ and twofold rotation symmetry $\left(d_{\|}=2\right)$, respectively.

\begin{tabular}{|c|c|c|c|c|c|c|c|c|}
\hline & & & $d=0$ & $d=1$ & $d=1$ & $d=2$ & $d=2$ & $d=2$ \\
\hline Class & $s$ & $t$ & $\mathcal{O}$ & $\mathcal{O}$ & $\mathcal{M}$ & $\mathcal{O}$ & $\mathcal{M}$ & $\mathcal{R}$ \\
\hline $\mathrm{AI}^{\mathcal{S}_{+}}$ & 0 & 0 & $\mathbb{Z}^{2}$ & $0 \subseteq 0$ & $\mathbb{Z} \subseteq \mathbb{Z}$ & $0 \subseteq 0 \subseteq 0$ & $0 \subseteq 0 \subseteq 0$ & $2 \mathbb{Z} \subseteq 2 \mathbb{Z} \subseteq 2 \mathbb{Z}$ \\
\hline $\mathrm{BDI}^{\mathcal{S}_{++}}$ & 1 & 0 & $\mathbb{Z}_{2}^{2}$ & $0 \subseteq \mathbb{Z}^{2}$ & $\mathbb{Z}_{2} \subseteq \mathbb{Z}_{2}$ & $0 \subseteq 0 \subseteq 0$ & $0 \subseteq \mathbb{Z} \subseteq \mathbb{Z}$ & $0 \subseteq 0 \subseteq 0$ \\
\hline $\mathrm{D}^{\mathcal{S}_{+}}$ & 2 & 0 & $\mathbb{Z}_{2}^{2}$ & $0 \subseteq \mathbb{Z}_{2}^{2}$ & $\mathbb{Z}_{2} \subseteq \mathbb{Z}_{2}$ & $0 \subseteq 0 \subseteq \mathbb{Z}^{2}$ & $0 \subseteq \mathbb{Z}_{2} \subseteq \mathbb{Z}_{2}$ & $0 \subseteq 0 \subseteq \mathbb{Z}$ \\
\hline $\mathrm{DIII}^{\mathcal{S}_{++}}$ & 3 & 0 & 0 & $0 \subseteq \mathbb{Z}_{2}^{2}$ & $0 \subseteq 0$ & $0 \subseteq 0 \subseteq \mathbb{Z}_{2}^{2}$ & $0 \subseteq \mathbb{Z}_{2} \subseteq \mathbb{Z}_{2}$ & $0 \subseteq 0 \subseteq 0$ \\
\hline $\mathrm{AII}^{\mathcal{S}_{+}}$ & 4 & 0 & $2 \mathbb{Z}^{2}$ & $0 \subseteq 0$ & $2 \mathbb{Z} \subseteq 2 \mathbb{Z}$ & $0 \subseteq 0 \subseteq \mathbb{Z}_{2}^{2}$ & $0 \subseteq 0 \subseteq 0$ & $4 \mathbb{Z} \subseteq 4 \mathbb{Z} \subseteq 2 \mathbb{Z}$ \\
\hline $\mathrm{CII}^{\mathcal{S}_{++}}$ & 5 & 0 & 0 & $0 \subseteq 2 \mathbb{Z}^{2}$ & $0 \subseteq 0$ & $0 \subseteq 0 \subseteq 0$ & $0 \subseteq 2 \mathbb{Z} \subseteq 2 \mathbb{Z}$ & $0 \subseteq 0 \subseteq 0$ \\
\hline $\mathrm{C}^{\mathcal{S}_{+}}$ & 6 & 0 & 0 & $0 \subseteq 0$ & $0 \subseteq 0$ & $0 \subseteq 0 \subseteq 2 \mathbb{Z}^{2}$ & $0 \subseteq 0 \subseteq 0$ & $0 \subseteq 0 \subseteq \mathbb{Z}$ \\
\hline $\mathrm{CI}^{\mathcal{S}_{++}}$ & 7 & 0 & 0 & $0 \subseteq 0$ & $0 \subseteq 0$ & $0 \subseteq 0 \subseteq 0$ & $0 \subseteq 0 \subseteq 0$ & $0 \subseteq 0 \subseteq 0$ \\
\hline $\mathrm{AI}^{\mathcal{C S} \mathcal{S}_{-}}$ & 0 & 1 & 0 & $0 \subseteq 0$ & $0 \subseteq 0$ & $0 \subseteq 0 \subseteq 0$ & $0 \subseteq 0 \subseteq 0$ & $0 \subseteq 0 \subseteq 0$ \\
\hline $\mathrm{BDI}^{\mathcal{S}_{+-}}$ & 1 & 1 & $\mathbb{Z}$ & $0 \subseteq 0$ & $\mathbb{Z} \subseteq \mathbb{Z}^{2}$ & $0 \subseteq 0 \subseteq 0$ & $0 \subseteq 0 \subseteq 0$ & $2 \mathbb{Z} \subseteq \mathbb{Z} \subseteq \mathbb{Z}$ \\
\hline $\mathrm{D}^{\mathcal{C S} \mathcal{S}_{+}}$ & 2 & 1 & $\mathbb{Z}_{2}$ & $0 \subseteq \mathbb{Z}$ & $\mathbb{Z}_{2} \subseteq \mathbb{Z}_{2}^{2}$ & $0 \subseteq 0 \subseteq 0$ & $0 \subseteq \mathbb{Z} \subseteq \mathbb{Z}^{2}$ & $0 \subseteq \mathbb{Z}_{2} \subseteq \mathbb{Z}_{2}$ \\
\hline $\mathrm{DIII}^{\mathcal{S}_{-+}}$ & 3 & 1 & $\mathbb{Z}_{2}$ & $0 \subseteq \mathbb{Z}_{2}$ & $\mathbb{Z}_{2} \subseteq \mathbb{Z}_{2}^{2}$ & $0 \subseteq 0 \subseteq \mathbb{Z}$ & $0 \subseteq \mathbb{Z}_{2} \subseteq \mathbb{Z}_{2}^{2}$ & $0 \subseteq \mathbb{Z}_{2} \subseteq \mathbb{Z}_{2}$ \\
\hline $\mathrm{AII}^{\mathcal{C S} \mathcal{S}_{-}}$ & 4 & 1 & 0 & $0 \subseteq \mathbb{Z}_{2}$ & $0 \subseteq 0$ & $0 \subseteq 0 \subseteq \mathbb{Z}_{2}$ & $0 \subseteq \mathbb{Z}_{2} \subseteq \mathbb{Z}_{2}^{2}$ & $0 \subseteq 0 \subseteq 0$ \\
\hline $\mathrm{CII}^{\mathcal{S}_{+-}}$ & 5 & 1 & $2 \mathbb{Z}$ & $0 \subseteq 0$ & $2 \mathbb{Z} \subseteq 2 \mathbb{Z}^{2}$ & $0 \subseteq 0 \subseteq \mathbb{Z}_{2}$ & $0 \subseteq 0 \subseteq 0$ & $4 \mathbb{Z} \subseteq 2 \mathbb{Z} \subseteq 2 \mathbb{Z}$ \\
\hline $\mathrm{C}^{\mathcal{C S}}{ }_{+}$ & 6 & 1 & 0 & $0 \subseteq 2 \mathbb{Z}$ & $0 \subseteq 0$ & $0 \subseteq 0 \subseteq 0$ & $0 \subseteq 2 \mathbb{Z} \subseteq 2 \mathbb{Z}^{2}$ & $0 \subseteq 0 \subseteq 0$ \\
\hline $\mathrm{CI}^{\mathcal{S}_{-+}}$ & 7 & 1 & 0 & $0 \subseteq 0$ & $0 \subseteq 0$ & $0 \subseteq 0 \subseteq 2 \mathbb{Z}$ & $0 \subseteq 0 \subseteq 0$ & $0 \subseteq 0 \subseteq 0$ \\
\hline $\mathrm{AI}^{\mathcal{S}_{-}}$ & 0 & 2 & $\mathbb{Z}$ & $0 \subseteq 0$ & $0 \subseteq 0$ & $0 \subseteq 0 \subseteq 2 \mathbb{Z}$ & $0 \subseteq 0 \subseteq 0$ & $0 \subseteq 0 \subseteq 0$ \\
\hline $\mathrm{BDI}^{\mathcal{S}_{--}}$ & 1 & 2 & 0 & $0 \subseteq \mathbb{Z}$ & $0 \subseteq 0$ & $0 \subseteq 0 \subseteq 0$ & $0 \subseteq 0 \subseteq 0$ & $0 \subseteq 0 \subseteq 0$ \\
\hline $\mathrm{D}^{\mathcal{S}_{-}}$ & 2 & 2 & $2 \mathbb{Z}$ & $0 \subseteq 0$ & $2 \mathbb{Z} \subseteq \mathbb{Z}$ & $0 \subseteq 0 \subseteq \mathbb{Z}$ & $0 \subseteq 0 \subseteq 0$ & $2 \mathbb{Z} \subseteq \mathbb{Z} \subseteq \mathbb{Z}^{2}$ \\
\hline DIII $^{\mathcal{S}_{--}}$ & 3 & 2 & 0 & $0 \subseteq 2 \mathbb{Z}$ & $0 \subseteq \mathbb{Z}_{2}$ & $0 \subseteq 0 \subseteq 0$ & $0 \subseteq 2 \mathbb{Z} \subseteq \mathbb{Z}$ & $0 \subseteq \mathbb{Z}_{2} \subseteq \mathbb{Z}_{2}^{2}$ \\
\hline $\mathrm{AII}^{\mathcal{S}_{-}}$ & 4 & 2 & $\mathbb{Z}$ & $0 \subseteq 0$ & $\mathbb{Z}_{2} \subseteq \mathbb{Z}_{2}$ & $0 \subseteq 0 \subseteq 2 \mathbb{Z}$ & $0 \subseteq 0 \subseteq \mathbb{Z}_{2}$ & $\mathbb{Z}_{2} \subseteq \mathbb{Z}_{2} \subseteq \mathbb{Z}_{2}^{2}$ \\
\hline $\mathrm{CII}^{\mathcal{S}_{--}}$ & 5 & 2 & 0 & $0 \subseteq \mathbb{Z}$ & $0 \subseteq 0$ & $0 \subseteq 0 \subseteq 0$ & $0 \subseteq \mathbb{Z}_{2} \subseteq \mathbb{Z}_{2}$ & $0 \subseteq 0 \subseteq 0$ \\
\hline $\mathrm{C}^{\mathcal{S}_{-}}$ & 6 & 2 & $2 \mathbb{Z}$ & $0 \subseteq 0$ & $2 \mathbb{Z} \subseteq 2 \mathbb{Z}$ & $0 \subseteq 0 \subseteq \mathbb{Z}$ & $0 \subseteq 0 \subseteq 0$ & $2 \mathbb{Z} \subseteq 2 \mathbb{Z} \subseteq 2 \mathbb{Z}^{2}$ \\
\hline $\mathrm{CI}^{\mathcal{S}_{--}}$ & 7 & 2 & 0 & $0 \subseteq 2 \mathbb{Z}$ & $0 \subseteq 0$ & $0 \subseteq 0 \subseteq 0$ & $0 \subseteq 2 \mathbb{Z} \subseteq 2 \mathbb{Z}$ & $0 \subseteq 0 \subseteq 0$ \\
\hline$\overline{\mathrm{AI}^{\mathcal{C S} \mathcal{S}_{+}}}$ & 0 & 3 & $\mathbb{Z}_{2}$ & $0 \subseteq \mathbb{Z}$ & $0 \subseteq 0$ & $0 \subseteq 0 \subseteq 0$ & $0 \subseteq 2 \mathbb{Z} \subseteq 2 \mathbb{Z}$ & $0 \subseteq 0 \subseteq 0$ \\
\hline $\mathrm{BDI}^{\mathcal{S}_{-+}}$ & 1 & 3 & $\mathbb{Z}_{2}$ & $0 \subseteq \mathbb{Z}_{2}$ & $0 \subseteq \mathbb{Z}$ & $0 \subseteq 0 \subseteq \mathbb{Z}$ & $0 \subseteq 0 \subseteq 0$ & $0 \subseteq 0 \subseteq 0$ \\
\hline $\mathrm{D}^{\mathcal{C S} \mathcal{S}_{-}}$ & 2 & 3 & 0 & $0 \subseteq \mathbb{Z}_{2}$ & $0 \subseteq 0$ & $0 \subseteq 0 \subseteq \mathbb{Z}_{2}$ & $0 \subseteq 0 \subseteq \mathbb{Z}$ & $0 \subseteq 0 \subseteq 0$ \\
\hline $\mathrm{DIII}^{\mathcal{S}_{+-}}$ & 3 & 3 & $2 \mathbb{Z}$ & $0 \subseteq 0$ & $4 \mathbb{Z} \subseteq 2 \mathbb{Z}$ & $0 \subseteq 0 \subseteq \mathbb{Z}_{2}$ & $0 \subseteq 0 \subseteq 0$ & $4 \mathbb{Z} \subseteq 2 \mathbb{Z} \subseteq \mathbb{Z}$ \\
\hline $\mathrm{AII}^{\mathcal{C} \mathcal{S}_{+}}$ & 4 & 3 & 0 & $0 \subseteq 2 \mathbb{Z}$ & $0 \subseteq 0$ & $0 \subseteq 0 \subseteq 0$ & $0 \subseteq 4 \mathbb{Z} \subseteq 2 \mathbb{Z}$ & $0 \subseteq 0 \subseteq \mathbb{Z}_{2}$ \\
\hline $\mathrm{CII}^{\mathcal{S}_{-+}}$ & 5 & 3 & 0 & $0 \subseteq 0$ & $0 \subseteq \mathbb{Z}$ & $0 \subseteq 0 \subseteq 2 \mathbb{Z}$ & $0 \subseteq 0 \subseteq 0$ & $0 \subseteq \mathbb{Z}_{2} \subseteq \mathbb{Z}_{2}$ \\
\hline $\mathrm{C}^{\mathcal{C S} \mathcal{S}_{-}}$ & 6 & 3 & 0 & $0 \subseteq 0$ & $0 \subseteq 0$ & $0 \subseteq 0 \subseteq 0$ & $0 \subseteq 0 \subseteq \mathbb{Z}$ & $0 \subseteq 0 \subseteq 0$ \\
\hline $\mathrm{CI}^{\mathcal{S}_{+-}}$ & 7 & 3 & $\mathbb{Z}$ & $0 \subseteq 0$ & $2 \mathbb{Z} \subseteq 2 \mathbb{Z}$ & $0 \subseteq 0 \subseteq 0$ & $0 \subseteq 0 \subseteq 0$ & $2 \mathbb{Z} \subseteq 2 \mathbb{Z} \subseteq 2 \mathbb{Z}$ \\
\hline
\end{tabular}

the corresponding classifying group. Formally, the group structure is obtained by the Grothendieck construction [6,57], where one considers equivalence classes of ordered pairs $\left(H_{1}, H_{2}\right)$ of Hamiltonians represented by Hermitian matrix-valued functions $H(\boldsymbol{k})$ of equal dimension, the equivalence relation being that two pairs $\left(H_{1}, H_{2}\right)$ and $\left(H_{1}^{\prime}, H_{2}^{\prime}\right)$ are topologically equivalent if $H_{1} \oplus H_{2}^{\prime}$ is continuously deformable to $H_{1}^{\prime} \oplus H_{2}$. Loosely speaking, the ordered pair $\left(H_{1}, H_{2}\right)$ represents the "difference" of the two Hamiltonians $H_{1}$ and $H_{2}$. Without loss of generality, one may take $H_{1}$ or $H_{2}$ to be a reference Hamiltonian $H_{\text {ref }}$. With this convention, the trivial element is represented by $\left(H_{\text {ref }}, H_{\text {ref }}\right)$, whereas the inverse of the group element $\left(H, H_{\text {ref }}\right)$ is $\left(H_{\text {ref }}, H\right)$. Alternatively, instead of the ordered pair $\left(H, H_{\text {ref }}\right)$ one may consider a one-parameter family of Hamiltonians $H(m)$ that interpolates between $H$ and the reference Hamiltonian $H_{\text {ref }}[22,58]$. In this work, we take the latter approach and consider the one-parameter family of Hamiltonians $H(m)$, such that $H(m)$ is in the topological class of $H$ for $-2<m<0$ and in the topological class of $H_{\text {ref }}$ for $0<m<2$, with the transition between topological classes (if any) taking place at $m=0$. When considering Hamiltonian families $H(m)$, we will often omit the parameter $m$ and refer to it simply as the "Hamiltonian $H$." The canonical-form Hamiltonians introduced in Sec. III are examples of such $m$-dependent families of Hamiltonians.

The classification of topological crystalline phases of Ref. [21] is based on isomorphisms between the groups 
$K\left(s, t \mid d, d_{\|}\right)$and $K\left(s \mid d, d_{\|}\right)$classifying $d$-dimensional Hamiltonians with the symmetries labeled by the corresponding indices, where $d_{\|}$is the number of inverted spatial dimensions. The above-mentioned isomorphisms are extensions of Teo and Kane's dimension-raising isomorphism [52] $\kappa$ increasing the spatial dimension by one to the systems with an order-two crystalline symmetry or antisymmetry [21]. Shiozaki and Sato introduce two isomorphisms $\kappa_{\|}$and $\kappa_{\perp}$, where the isomorphism $\kappa_{\|}$increases both the spatial dimension $d$ and the number of the inverted momenta $d_{\|}$, whereas the isomorphism $\kappa_{\perp}$ increases only the spatial dimension $d$ while keeping $d_{\|}$unchanged.

For the complex and real classes with unitary (anti) symmetry the classifying groups are denoted $K\left(s, t \mid d, d_{\|}\right)$ and these isomorphisms are (with $d_{\|}<d$ )

$$
\begin{gathered}
K\left(s, t \mid d, d_{\|}\right) \stackrel{\kappa_{\|}}{=} K\left(s+1, t+1 \mid d+1, d_{\|}+1\right) \\
\stackrel{\kappa_{\perp}}{=} K\left(s+1, t \mid d+1, d_{\|}\right),
\end{gathered}
$$

with the integers $s$ and $t$ taken mod 2 for complex classes, and $\bmod 8$ and $\bmod 4$, respectively, for the real classes. We use the same notation for the classifying groups for the real and complex classes. When discussing specific examples we will always specify the tenfold-way class using its Cartan symbol, so that no confusion is possible. For complex classes with antiunitary (anti)symmetry these isomorphisms are

$$
\begin{gathered}
K\left(s \mid d, d_{\|}\right) \stackrel{\kappa_{\|}}{=} K\left(s-1 \mid d+1, d_{\|}+1\right) \\
\stackrel{\kappa_{\Perp}}{=} K\left(s+1 \mid d+1, d_{\|}\right) .
\end{gathered}
$$

When applied repeatedly, these isomorphisms can be used to relate the classification problem of $d$-dimensional Hamiltonians with an order-two crystalline symmetry to a zerodimensional classification problem with an on-site symmetry $[21,25]$, which can be solved with elementary methods.

Following Teo and Kane, Shiozaki and Sato also introduce an isomorphism $\rho_{\|}$relating a topological class of Hamiltonians $H(\boldsymbol{k})$ with an additional crystalline (anti)symmetry $\mathcal{S}$ to the topological class of one-parameter family of Hamiltonians $H(\boldsymbol{k}, \varphi), 0 \leq \varphi \leq 2 \pi$, with the additional conditions $H(\boldsymbol{k}, 0)=H(\boldsymbol{k}, 2 \pi)$ and $H(\boldsymbol{k}, \varphi)=\mathcal{S} H(\boldsymbol{k}, 2 \pi-\varphi)$. This isomorphism and the dimension-raising isomorphism $\kappa_{\|}$ introduced above play a central role in our algebraic construction of a higher-order bulk-boundary correspondence for topological crystalline phases; see Secs. IV and V. Further details of these isomorphisms are given in Appendix B.

The Shiozaki-Sato classifying groups $K$ are the largest groups in the sequence (1), which for crystals of dimension $d=0,1$, and 2 are listed in Tables II-IV for the complex tenfold-way classes with unitary (anti)symmetries, the complex tenfold-way classes with antiunitary (anti)symmetries, and the real tenfold-way classes with unitary (anti) symmetries, respectively. The corresponding classification of three-dimensional systems is given in Tables V-VII. When no confusion is possible, we will omit the arguments $s$ and $t$ in what follows, and write $K\left(d, d_{\|}\right)$instead of $K\left(s, t \mid d, d_{\|}\right)$or $K\left(s \mid d, d_{\|}\right)$.

The 44 Shiozaki-Sato classes represent all mathematically possible algebraic relations between a twofold crystalline symmetry or antisymmetry and the fundamental nonspatial symmetries $\mathcal{T}, \mathcal{P}$, and $\mathcal{C}$. Not all of these classes are naturally realized in crystals, however. One important reason why it is, nevertheless, important to classify all mathematically allowed possibilities is the existence of the isomorphisms (6) and (7), which connect different symmetry classes in different dimensions. Another reason is that symmetry classes which at first sight may appear "unphysical" may be realized in condensed matter systems as effective symmetries; see, e.g., the examples presented in Refs. [32,59-61]. To facilitate the translation between the Shiozaki-Sato classes used in this article and the "physical" symmetries of crystals, Tables VIII and IX list the relevant Shiozaki-Sato classes for crystals with mirror, twofold rotation, or inversion symmetry. Here, we note that, whereas the physical inversion symmetry does not affect the spin d.o.f., the physical mirror and twofold rotation operations do. With our convention that (unitary) symmetries square to one, this implies that inversion commutes with the timereversal operation $\mathcal{T}$, whereas mirror and twofold rotation anticommute with $\mathcal{T}$ in a crystal with strong spin-orbit coupling. For a superconducting system with an order-two crystalline symmetry, a crystalline symmetry $\mathcal{S}$ must only leave the normal-state Hamiltonian unchanged, whereas the superconducting order parameter $\Delta$ may eventually change sign under $\mathcal{S}$. The parity of $\Delta$ under $\mathcal{S}$ determines whether $\mathcal{S}$ commutes or anticommutes with particle-hole conjugation.

TABLE V. Bulk classification sequence (1) for three-dimensional topological crystalline phases with an order-two unitary crystalline (anti)symmetry for the complex tenfold-way classes. The symbols $\mathcal{O}, \mathcal{M}$, $\mathcal{R}$, and $\mathcal{I}$ refer to local on-site $\left(d_{\|}=0\right)$, mirror $\left(d_{\|}=1\right)$, twofold rotation $\left(d_{\|}=2\right)$, and inversion symmetry $\left(d_{\|}=3\right)$, respectively.

\begin{tabular}{lllcccc}
\hline \hline Class & $s$ & $t$ & $\mathcal{O}$ & $\mathcal{M}$ & $\mathcal{R}$ & $\mathcal{I}$ \\
\hline $\mathrm{A}^{\mathcal{S}}$ & 0 & 0 & $0 \subseteq 0 \subseteq 0 \subseteq 0$ & $0 \subseteq 0 \subseteq \mathbb{Z} \subseteq \mathbb{Z}$ & $0 \subseteq 0 \subseteq 0 \subseteq 0$ & $2 \mathbb{Z} \subseteq 2 \mathbb{Z} \subseteq \mathbb{Z} \subseteq \mathbb{Z}$ \\
$\mathrm{AIII}^{\mathcal{S}_{+}}$ & 1 & 0 & $0 \subseteq 0 \subseteq 0 \subseteq \mathbb{Z}^{2}$ & $0 \subseteq 0 \subseteq 0 \subseteq 0$ & $0 \subseteq \mathbb{Z} \subseteq \mathbb{Z} \subseteq \mathbb{Z}^{2}$ & $0 \subseteq 0 \subseteq 0 \subseteq 0$ \\
\hline $\mathrm{A}^{\mathcal{C S}}$ & 0 & 1 & $0 \subseteq 0 \subseteq 0 \subseteq \mathbb{Z}$ & $0 \subseteq 0 \subseteq 0 \subseteq 0$ & $0 \subseteq 2 \mathbb{Z} \subseteq \mathbb{Z} \subseteq \mathbb{Z}$ & $0 \subseteq 0 \subseteq 0 \subseteq 0$ \\
$\mathrm{AIII}^{\mathcal{S}_{-}}$ & 1 & 1 & $0 \subseteq 0 \subseteq 0 \subseteq 0$ & $0 \subseteq 0 \subseteq \mathbb{Z} \subseteq \mathbb{Z}^{2}$ & $0 \subseteq 0 \subseteq 0 \subseteq 0$ & $2 \mathbb{Z} \subseteq \mathbb{Z} \subseteq \mathbb{Z} \subseteq \mathbb{Z}$ \\
\hline \hline
\end{tabular}


TABLE VI. Same as Table V, but for antiunitary (anti)symmetries.

\begin{tabular}{llcccc}
\hline \hline Class & $s$ & $\mathcal{O}$ & $\mathcal{M}$ & $\mathcal{R}$ & $\mathcal{I}$ \\
\hline $\mathrm{A}^{\mathcal{T}^{+} \mathcal{S}}$ & 0 & $0 \subseteq 0 \subseteq 0 \subseteq 0$ & $0 \subseteq 0 \subseteq 0 \subseteq 0$ & $0 \subseteq 0 \subseteq \mathbb{Z}_{2} \subseteq \mathbb{Z}_{2}$ & $0 \subseteq 0 \subseteq 0 \subseteq 0$ \\
$\mathrm{AIII}^{\mathcal{P}^{+} \mathcal{S}_{+}}$ & 1 & $0 \subseteq 0 \subseteq 0 \subseteq 0$ & $0 \subseteq 0 \subseteq 0 \subseteq \mathbb{Z}$ & $0 \subseteq \mathbb{Z}_{2} \subseteq \mathbb{Z}_{2} \subseteq \mathbb{Z}_{2}$ & $0 \subseteq 0 \subseteq 0 \subseteq 2 \mathbb{Z}$ \\
$\mathrm{A}^{\mathcal{P}^{+} \mathcal{S}}$ & 2 & $0 \subseteq 0 \subseteq 0 \subseteq 0$ & $0 \subseteq 0 \subseteq \mathbb{Z}_{2} \subseteq \mathbb{Z}_{2}$ & $0 \subseteq 0 \subseteq 0 \subseteq 0$ & $0 \subseteq 0 \subseteq 0 \subseteq 0$ \\
$\mathrm{AIII}^{\mathcal{T}^{-} \mathcal{S}_{-}}$ & 3 & $0 \subseteq 0 \subseteq 0 \subseteq \mathbb{Z}$ & $0 \subseteq 0 \subseteq \mathbb{Z}_{2} \subseteq \mathbb{Z}_{2}$ & $0 \subseteq 0 \subseteq 0 \subseteq 2 \mathbb{Z}$ & $0 \subseteq 0 \subseteq 0 \subseteq 0$ \\
$\mathrm{~A}^{\mathcal{T}^{-} \mathcal{S}}$ & 4 & $0 \subseteq 0 \subseteq 0 \subseteq \mathbb{Z}_{2}$ & $0 \subseteq 0 \subseteq 0 \subseteq 0$ & $0 \subseteq 0 \subseteq 0 \subseteq 0$ & $0 \subseteq 0 \subseteq 0 \subseteq 0$ \\
$\mathrm{AIII}^{\mathcal{P}^{-} \mathcal{S}_{+}}$ & 5 & $0 \subseteq 0 \subseteq 0 \subseteq \mathbb{Z}_{2}$ & $0 \subseteq 0 \subseteq 0 \subseteq 2 \mathbb{Z}$ & $0 \subseteq 0 \subseteq 0 \subseteq 0$ & $0 \subseteq 0 \subseteq 0 \subseteq \mathbb{Z}$ \\
$\mathrm{A}^{\mathcal{P}^{-} \mathcal{S}}$ & 6 & $0 \subseteq 0 \subseteq 0 \subseteq 0$ & $0 \subseteq 0 \subseteq 0 \subseteq 0$ & $0 \subseteq 0 \subseteq 0 \subseteq 0$ & $0 \subseteq 0 \subseteq \mathbb{Z}_{2} \subseteq \mathbb{Z}_{2}$ \\
$\mathrm{AIII}^{\mathcal{T}^{+} \mathcal{S}_{-}}$ & 7 & $0 \subseteq 0 \subseteq 0 \subseteq 2 \mathbb{Z}$ & $0 \subseteq 0 \subseteq 0 \subseteq 0$ & $0 \subseteq 0 \subseteq 0 \subseteq \mathbb{Z}$ & $0 \subseteq \mathbb{Z}_{2} \subseteq \mathbb{Z}_{2} \subseteq \mathbb{Z}_{2}$ \\
\hline \hline
\end{tabular}

TABLE VII. Bulk classification sequence (1) for three-dimensional topological crystalline phases with an ordertwo crystalline symmetry or antisymmetry for the real tenfold-way classes. The symbols $\mathcal{O}, \mathcal{M}, \mathcal{R}$, and $\mathcal{I}$ refer to local on-site $\left(d_{\|}=0\right)$, mirror $\left(d_{\|}=1\right)$, twofold rotation $\left(d_{\|}=2\right)$, and inversion symmetry $\left(d_{\|}=3\right)$, respectively.

\begin{tabular}{|c|c|c|c|c|c|c|}
\hline Class & $s$ & $t$ & $\mathcal{O}$ & $\mathcal{M}$ & $\mathcal{R}$ & $\mathcal{I}$ \\
\hline $\mathrm{AI}^{\mathcal{S}_{+}}$ & 0 & 0 & $0 \subseteq 0 \subseteq 0 \subseteq 0$ & $0 \subseteq 0 \subseteq 0 \subseteq 0$ & $0 \subseteq 0 \subseteq 0 \subseteq 0$ & $2 \mathbb{Z} \subseteq 2 \mathbb{Z} \subseteq 2 \mathbb{Z} \subseteq 2 \mathbb{Z}$ \\
\hline $\mathrm{BDI}^{\mathcal{S}_{++}}$ & 1 & 0 & $0 \subseteq 0 \subseteq 0 \subseteq 0$ & $0 \subseteq 0 \subseteq 0 \subseteq 0$ & $0 \subseteq 2 \mathbb{Z} \subseteq 2 \mathbb{Z} \subseteq 2 \mathbb{Z}$ & $0 \subseteq 0 \subseteq 0 \subseteq 0$ \\
\hline $\mathrm{D}^{\mathcal{S}_{+}}$ & 2 & 0 & $0 \subseteq 0 \subseteq 0 \subseteq 0$ & $0 \subseteq 0 \subseteq \mathbb{Z} \subseteq \mathbb{Z}$ & $0 \subseteq 0 \subseteq 0 \subseteq 0$ & $0 \subseteq 0 \subseteq 0 \subseteq 0$ \\
\hline $\mathrm{DIII}^{\mathcal{S}_{++}}$ & 3 & 0 & $0 \subseteq 0 \subseteq 0 \subseteq \mathbb{Z}^{2}$ & $0 \subseteq 0 \subseteq \mathbb{Z}_{2} \subseteq \mathbb{Z}_{2}$ & $0 \subseteq 0 \subseteq 0 \subseteq \mathbb{Z}$ & $0 \subseteq 0 \subseteq 0 \subseteq 0$ \\
\hline $\mathrm{AII}^{\mathcal{S}_{+}}$ & 4 & 0 & $0 \subseteq 0 \subseteq 0 \subseteq \mathbb{Z}_{2}^{2}$ & $0 \subseteq 0 \subseteq \mathbb{Z}_{2} \subseteq \mathbb{Z}_{2}$ & $0 \subseteq 0 \subseteq 0 \subseteq 0$ & $4 \mathbb{Z} \subseteq 4 \mathbb{Z} \subseteq 2 \mathbb{Z} \subseteq \mathbb{Z}$ \\
\hline $\mathrm{CII}^{\mathcal{S}_{++}}$ & 5 & 0 & $0 \subseteq 0 \subseteq 0 \subseteq \mathbb{Z}_{2}^{2}$ & $0 \subseteq 0 \subseteq 0 \subseteq 0$ & $0 \subseteq 4 \mathbb{Z} \subseteq 4 \mathbb{Z} \subseteq 2 \mathbb{Z}$ & $0 \subseteq 0 \subseteq 0 \subseteq \mathbb{Z}_{2}$ \\
\hline $\mathrm{C}^{\mathcal{S}_{+}}$ & 6 & 0 & $0 \subseteq 0 \subseteq 0 \subseteq 0$ & $0 \subseteq 0 \subseteq 2 \mathbb{Z} \subseteq 2 \mathbb{Z}$ & $0 \subseteq 0 \subseteq 0 \subseteq 0$ & $0 \subseteq 0 \subseteq \mathbb{Z}_{2} \subseteq \mathbb{Z}_{2}$ \\
\hline $\mathrm{CI}^{\mathcal{S}_{++}}$ & 7 & 0 & $0 \subseteq 0 \subseteq 0 \subseteq 2 \mathbb{Z}^{2}$ & $0 \subseteq 0 \subseteq 0 \subseteq 0$ & $0 \subseteq 0 \subseteq 0 \subseteq \mathbb{Z}$ & $0 \subseteq 0 \subseteq 0 \subseteq 0$ \\
\hline $\mathrm{AI}^{\mathcal{C S} \mathcal{S}_{-}}$ & 0 & 1 & $0 \subseteq 0 \subseteq 0 \subseteq 2 \mathbb{Z}$ & $0 \subseteq 0 \subseteq 0 \subseteq 0$ & $0 \subseteq 0 \subseteq 0 \subseteq 0$ & $0 \subseteq 0 \subseteq 0 \subseteq 0$ \\
\hline $\mathrm{BDI}^{\mathcal{S}_{+-}}$ & 1 & 1 & $0 \subseteq 0 \subseteq 0 \subseteq 0$ & $0 \subseteq 0 \subseteq 0 \subseteq 0$ & $0 \subseteq 0 \subseteq 0 \subseteq 0$ & $4 \mathbb{Z} \subseteq 2 \mathbb{Z} \subseteq 2 \mathbb{Z} \subseteq 2 \mathbb{Z}$ \\
\hline $\mathrm{D}^{\mathcal{C S} \mathcal{S}_{+}}$ & 2 & 1 & $0 \subseteq 0 \subseteq 0 \subseteq 0$ & $0 \subseteq 0 \subseteq 0 \subseteq 0$ & $0 \subseteq 2 \mathbb{Z} \subseteq \mathbb{Z} \subseteq \mathbb{Z}$ & $0 \subseteq 0 \subseteq 0 \subseteq 0$ \\
\hline $\mathrm{DIII}^{\mathcal{S}_{-+}}$ & 3 & 1 & $0 \subseteq 0 \subseteq 0 \subseteq 0$ & $0 \subseteq 0 \subseteq \mathbb{Z} \subseteq \mathbb{Z}^{2}$ & $0 \subseteq 0 \subseteq \mathbb{Z}_{2} \subseteq \mathbb{Z}_{2}$ & $0 \subseteq 0 \subseteq 0 \subseteq \mathbb{Z}$ \\
\hline $\mathrm{AII}^{\mathcal{C} \mathcal{S}_{-}}$ & 4 & 1 & $0 \subseteq 0 \subseteq 0 \subseteq \mathbb{Z}$ & $0 \subseteq 0 \subseteq \mathbb{Z}_{2} \subseteq \mathbb{Z}_{2}^{2}$ & $0 \subseteq 0 \subseteq \mathbb{Z}_{2} \subseteq \mathbb{Z}_{2}$ & $0 \subseteq 0 \subseteq 0 \subseteq 0$ \\
\hline $\mathrm{CII}^{\mathcal{S}_{+-}}$ & 5 & 1 & $0 \subseteq 0 \subseteq 0 \subseteq \mathbb{Z}_{2}$ & $0 \subseteq 0 \subseteq \mathbb{Z}_{2} \subseteq \mathbb{Z}_{2}^{2}$ & $0 \subseteq 0 \subseteq 0 \subseteq 0$ & $8 \mathbb{Z} \subseteq 4 \mathbb{Z} \subseteq 4 \mathbb{Z} \subseteq 2 \mathbb{Z}$ \\
\hline $\mathrm{C}^{\mathcal{C S} \mathcal{S}_{+}}$ & 6 & 1 & $0 \subseteq 0 \subseteq 0 \subseteq \mathbb{Z}_{2}$ & $0 \subseteq 0 \subseteq 0 \subseteq 0$ & $0 \subseteq 4 \mathbb{Z} \subseteq 2 \mathbb{Z} \subseteq 2 \mathbb{Z}$ & $0 \subseteq 0 \subseteq 0 \subseteq 0$ \\
\hline $\mathrm{CI}^{\mathcal{S}_{-+}}$ & 7 & 1 & $0 \subseteq 0 \subseteq 0 \subseteq 0$ & $0 \subseteq 0 \subseteq 2 \mathbb{Z} \subseteq 2 \mathbb{Z}^{2}$ & $0 \subseteq 0 \subseteq 0 \subseteq 0$ & $0 \subseteq 0 \subseteq 0 \subseteq \mathbb{Z}$ \\
\hline $\mathrm{AI}^{\mathcal{S}_{-}}$ & 0 & 2 & $0 \subseteq 0 \subseteq 0 \subseteq 0$ & $0 \subseteq 0 \subseteq 2 \mathbb{Z} \subseteq 2 \mathbb{Z}$ & $0 \subseteq 0 \subseteq 0 \subseteq 0$ & $0 \subseteq 0 \subseteq 0 \subseteq 0$ \\
\hline $\mathrm{BDI}^{\mathcal{S}_{--}}$ & 1 & 2 & $0 \subseteq 0 \subseteq 0 \subseteq 2 \mathbb{Z}$ & $0 \subseteq 0 \subseteq 0 \subseteq 0$ & $0 \subseteq 0 \subseteq 0 \subseteq 0$ & $0 \subseteq 0 \subseteq 0 \subseteq 0$ \\
\hline $\mathrm{D}^{\mathcal{S}_{-}}$ & 2 & 2 & $0 \subseteq 0 \subseteq 0 \subseteq 0$ & $0 \subseteq 0 \subseteq 0 \subseteq 0$ & $0 \subseteq 0 \subseteq 0 \subseteq 0$ & $4 \mathbb{Z} \subseteq 2 \mathbb{Z} \subseteq \mathbb{Z} \subseteq \mathbb{Z}$ \\
\hline DIII $^{\mathcal{S}_{--}}$ & 3 & 2 & $0 \subseteq 0 \subseteq 0 \subseteq \mathbb{Z}$ & $0 \subseteq 0 \subseteq 0 \subseteq 0$ & $0 \subseteq 2 \mathbb{Z} \subseteq \mathbb{Z} \subseteq \mathbb{Z}^{2}$ & $0 \subseteq 0 \subseteq \mathbb{Z}_{2} \subseteq \mathbb{Z}_{2}$ \\
\hline $\mathrm{AII}^{\mathcal{S}_{-}}$ & 4 & 2 & $0 \subseteq 0 \subseteq 0 \subseteq 0$ & $0 \subseteq 0 \subseteq 2 \mathbb{Z} \subseteq \mathbb{Z}$ & $0 \subseteq 0 \subseteq \mathbb{Z}_{2} \subseteq \mathbb{Z}_{2}^{2}$ & $0 \subseteq 0 \subseteq \mathbb{Z}_{2} \subseteq \mathbb{Z}_{2}$ \\
\hline $\mathrm{CII}^{\mathcal{S}_{--}}$ & 5 & 2 & $0 \subseteq 0 \subseteq 0 \subseteq 2 \mathbb{Z}$ & $0 \subseteq 0 \subseteq 0 \subseteq \mathbb{Z}_{2}$ & $0 \subseteq \mathbb{Z}_{2} \subseteq \mathbb{Z}_{2} \subseteq \mathbb{Z}_{2}^{2}$ & $0 \subseteq 0 \subseteq 0 \subseteq 0$ \\
\hline $\mathrm{C}^{\mathcal{S}_{-}}$ & 6 & 2 & $0 \subseteq 0 \subseteq 0 \subseteq 0$ & $0 \subseteq 0 \subseteq \mathbb{Z}_{2} \subseteq \mathbb{Z}_{2}$ & $0 \subseteq 0 \subseteq 0 \subseteq 0$ & $4 \mathbb{Z} \subseteq 4 \mathbb{Z} \subseteq 2 \mathbb{Z} \subseteq 2 \mathbb{Z}$ \\
\hline $\mathrm{CI}^{\mathcal{S}_{--}}$ & 7 & 2 & $0 \subseteq 0 \subseteq 0 \subseteq \mathbb{Z}$ & $0 \subseteq 0 \subseteq 0 \subseteq 0$ & $0 \subseteq 2 \mathbb{Z} \subseteq 2 \mathbb{Z} \subseteq 2 \mathbb{Z}^{2}$ & $0 \subseteq 0 \subseteq 0 \subseteq 0$ \\
\hline $\mathrm{AI}^{\mathcal{C S} \mathcal{S}_{+}}$ & 0 & 3 & $0 \subseteq 0 \subseteq 0 \subseteq 0$ & $0 \subseteq 0 \subseteq 0 \subseteq 0$ & $0 \subseteq 2 \mathbb{Z} \subseteq 2 \mathbb{Z} \subseteq 2 \mathbb{Z}$ & $0 \subseteq 0 \subseteq 0 \subseteq 0$ \\
\hline $\mathrm{BDI}^{\mathcal{S}_{-+}}$ & 1 & 3 & $0 \subseteq 0 \subseteq 0 \subseteq 0$ & $0 \subseteq 0 \subseteq 2 \mathbb{Z} \subseteq 2 \mathbb{Z}$ & $0 \subseteq 0 \subseteq 0 \subseteq 0$ & $0 \subseteq 0 \subseteq 0 \subseteq 0$ \\
\hline $\mathrm{D}^{\mathcal{C S} \mathcal{S}_{-}}$ & 2 & 3 & $0 \subseteq 0 \subseteq 0 \subseteq \mathbb{Z}$ & $0 \subseteq 0 \subseteq 0 \subseteq 0$ & $0 \subseteq 0 \subseteq 0 \subseteq 0$ & $0 \subseteq 0 \subseteq 0 \subseteq 0$ \\
\hline $\mathrm{DIII}^{\mathcal{S}_{+-}}$ & 3 & 3 & $0 \subseteq 0 \subseteq 0 \subseteq \mathbb{Z}_{2}$ & $0 \subseteq 0 \subseteq 0 \subseteq \mathbb{Z}$ & $0 \subseteq 0 \subseteq 0 \subseteq 0$ & $4 \mathbb{Z} \subseteq 2 \mathbb{Z} \subseteq \mathbb{Z} \subseteq \mathbb{Z}^{2}$ \\
\hline $\mathrm{AII}^{\mathcal{C} \mathcal{S}_{+}}$ & 4 & 3 & $0 \subseteq 0 \subseteq 0 \subseteq \mathbb{Z}_{2}$ & $0 \subseteq 0 \subseteq 0 \subseteq 0$ & $0 \subseteq 4 \mathbb{Z} \subseteq 2 \mathbb{Z} \subseteq \mathbb{Z}$ & $0 \subseteq 0 \subseteq \mathbb{Z}_{2} \subseteq \mathbb{Z}_{2}^{2}$ \\
\hline $\mathrm{CII}^{\mathcal{S}_{-+}}$ & 5 & 3 & $0 \subseteq 0 \subseteq 0 \subseteq 0$ & $0 \subseteq 0 \subseteq 4 \mathbb{Z} \subseteq 2 \mathbb{Z}$ & $0 \subseteq 0 \subseteq 0 \subseteq \mathbb{Z}_{2}$ & $0 \subseteq \mathbb{Z}_{2} \subseteq \mathbb{Z}_{2} \subseteq \mathbb{Z}_{2}^{2}$ \\
\hline $\mathrm{C}^{\mathcal{C \mathcal { S } _ { - }}}$ & 6 & 3 & $0 \subseteq 0 \subseteq 0 \subseteq 2 \mathbb{Z}$ & $0 \subseteq 0 \subseteq 0 \subseteq 0$ & $0 \subseteq 0 \subseteq \mathbb{Z}_{2} \subseteq \mathbb{Z}_{2}$ & $0 \subseteq 0 \subseteq 0 \subseteq 0$ \\
\hline $\mathrm{CI}^{\mathcal{S}_{+-}}$ & 7 & 3 & $0 \subseteq 0 \subseteq 0 \subseteq 0$ & $0 \subseteq 0 \subseteq 0 \subseteq \mathbb{Z}$ & $0 \subseteq 0 \subseteq 0 \subseteq 0$ & $2 \mathbb{Z} \subseteq 2 \mathbb{Z} \subseteq 2 \mathbb{Z} \subseteq 2 \mathbb{Z}^{2}$ \\
\hline
\end{tabular}


(Please note that there are physical symmetries not included in Tables VIII and IX, such as magnetic symmetries.)

The classifying groups for the tenfold-way classes (i.e., without additional crystalline symmetries) are denoted by $K_{\mathrm{TF}}(s \mid d)$. (See Table I for the symmetry label $s$; the symmetry label $t$ does not apply to the tenfold-way classes.) We further define the subgroup

$$
K_{\mathrm{TF}, \mathcal{S}}(s, t \mid d) \subseteq K_{\mathrm{TF}}(s \mid d)
$$

which consists of those tenfold-way phases that are compatible with the crystalline (anti)symmetry $\mathcal{S}$. Since tenfold-way phases are first-order topological phases and since the (anti)symmetry $\mathcal{S}$ is a nonlocal symmetry at a generic boundary for $d_{\|} \geq 1$, for $d_{\|} \geq 1$ we have the isomorphism

$$
K_{\mathrm{TF}, \mathcal{S}}(s, t \mid d)=K\left(s, t \mid d, d_{\|}\right) / K^{\prime}\left(s, t \mid d, d_{\|}\right),
$$

which identifies the quotient group $K\left(s, t \mid d, d_{\|}\right) / K^{\prime}\left(s, t \mid d, d_{\|}\right)$ as a regular subgroup of $K_{\mathrm{TF}}(s \mid d)$ for $d_{\|} \geq 1$. No such isomorphism exists if $d_{\|}=0$ because in that case $\mathcal{S}$ is a local symmetry at a generic crystal boundary, allowing for a richer boundary classifying group than the one obtained from the tenfold-way classification.

The tenfold-way classification and the Shiozaki-Sato classification of topological phases with a crystalline ordertwo symmetry contains only "strong" topological crystalline invariants, i.e., they address topological features that are unaffected by resizing of the unit cell, thus allowing the addition of perturbations that break the translation symmetry of the original (smaller) unit cell, while preserving the crystalline symmetries. Throughout this work we only consider HOTPs originating from such strong topology.

TABLE VIII. Shiozaki-Sato classes that correspond to natural physical realizations of the order-two symmetries for insulators. The top and bottom panels are for crystals with and without strong spin-orbit coupling, respectively. Time-reversal-symmetric insulators have tenfold-way class AII or AI, otherwise the class

\begin{tabular}{|c|c|c|c|}
\hline Spin orbit & $d=1$ & $d=2$ & $d=3$ \\
\hline $\begin{array}{l}\text { Mirror } \\
\text { Twofold rotation } \\
\text { Inversion }\end{array}$ & $\begin{array}{l}\mathrm{A}^{\mathcal{M}}, \mathrm{AII}^{\mathcal{M}_{-}} \\
\mathrm{A}^{\mathcal{M}}, \mathrm{AII}^{\mathcal{M}_{-}} \\
\mathrm{A}^{\mathcal{M}}, \mathrm{AII}^{\mathcal{M}_{+}}\end{array}$ & $\begin{array}{l}\mathrm{A}^{\mathcal{M}}, \mathrm{AII}^{\mathcal{M}}{ }_{-} \\
\mathrm{A}^{\mathcal{R}}, \mathrm{AII}^{\mathcal{R}_{-}} \\
\mathrm{A}^{\mathcal{R}}, \mathrm{AII}^{\mathcal{R}_{+}}\end{array}$ & $\begin{array}{l}\mathrm{A}^{\mathcal{M}}, \mathrm{AII}^{\mathcal{M}_{-}} \\
\mathrm{A}^{\mathcal{R}}, \mathrm{AII}^{\mathcal{R}_{-}} \\
\mathrm{A}^{\mathcal{I}}, \mathrm{AII}^{\mathcal{I}_{+}}\end{array}$ \\
\hline No spin orbit & $d=1$ & $d=2$ & $d=3$ \\
\hline $\begin{array}{l}\text { Mirror } \\
\text { Twofold rotation } \\
\text { Inversion } \\
\end{array}$ & $\begin{array}{l}\mathrm{A}^{\mathcal{M}}, \mathrm{AI}^{\mathcal{M}}{ }_{+} \\
\mathrm{A}^{\mathcal{M}}, \mathrm{AI}^{\mathcal{M}}{ }_{+} \\
\mathrm{A}^{\mathcal{M}}, \mathrm{AI}^{\mathcal{M}+}\end{array}$ & $\begin{array}{l}\mathrm{A}^{\mathcal{M}}, \mathrm{AI}^{\mathcal{M}_{+}} \\
\mathrm{A}^{\mathcal{R}}, \mathrm{AI}^{\mathcal{R}_{+}} \\
\mathrm{A}^{\mathcal{R}}, \mathrm{AI}^{\mathcal{R}_{+}}\end{array}$ & $\begin{array}{c}\mathrm{A}^{\mathcal{M}}, \mathrm{AI}^{\mathcal{M}_{+}} \\
\mathrm{A}^{\mathcal{R}}, \mathrm{AI}^{\mathcal{R}_{+}} \\
\mathrm{A}^{\mathcal{I}}, \mathrm{AI}^{\mathcal{I}_{+}}\end{array}$ \\
\hline
\end{tabular}
is $\mathrm{A}$.
TABLE IX. Shiozaki-Sato classes that correspond to natural physical realizations of the order-two symmetries for superconductors with (top) and without (bottom) strong spin-orbit coupling. For the time-reversal invariant superconductors the tenfold-way class is DIII or CI, otherwise it is class D or C. The parity of the superconducting order parameter under the ordertwo symmetry $\mathcal{S}$ is denoted $\alpha= \pm$. For classes $\mathrm{C}$ and $\mathrm{CI}$ we

\begin{tabular}{|c|c|c|c|}
\hline Spin orbit & $d=1$ & $d=2$ & $d=3$ \\
\hline $\begin{array}{l}\text { Mirror } \\
\text { Rotation } \\
\text { Inversion }\end{array}$ & $\begin{array}{l}\mathrm{D}^{\mathcal{M}_{\alpha}}, \mathrm{DIII}^{\mathcal{M}_{-\alpha}} \\
\mathrm{D}^{\mathcal{M}_{\alpha}}, \mathrm{DIII}^{\mathcal{M}_{-\alpha}} \\
\mathrm{D}^{\mathcal{M}_{\alpha}}, \mathrm{DIII}^{\mathcal{M}_{+\alpha}}\end{array}$ & $\begin{array}{l}\mathrm{D}^{\mathcal{M}_{\alpha}}, \mathrm{DIII}^{\mathcal{M}_{-\alpha}} \\
\mathrm{D}^{\mathcal{R}_{\alpha}}, \mathrm{DIIII}^{\mathcal{R}_{-\alpha}} \\
\mathrm{D}^{\mathcal{R}_{\alpha}}, \mathrm{DIII}^{\mathcal{R}_{+\alpha}}\end{array}$ & $\begin{array}{l}\mathrm{D}^{\mathcal{M}_{\alpha}}, \mathrm{DIII}^{\mathcal{M}_{-\alpha}} \\
\mathrm{D}^{\mathcal{R}_{\alpha}}, \mathrm{DIII}^{\mathcal{R}_{-\alpha}} \\
\mathrm{D}^{\mathcal{I}_{\alpha}}, \mathrm{DIII}^{\mathcal{I}_{+\alpha}}\end{array}$ \\
\hline No spin orbit & $d=1$ & $d=2$ & $d=3$ \\
\hline $\begin{array}{l}\text { Mirror } \\
\text { Rotation } \\
\text { Inversion }\end{array}$ & $\begin{array}{l}\mathrm{C}^{\mathcal{M}_{\alpha}}, \mathrm{CI}^{\mathcal{M}_{+\alpha}} \\
\mathrm{C}^{\mathcal{M}_{\alpha}}, \mathrm{CI}^{\mathcal{M}_{+\alpha}} \\
\mathrm{C}^{\mathcal{M}_{\alpha}}, \mathrm{CI}^{\mathcal{M}_{+\alpha}}\end{array}$ & $\begin{array}{l}\mathrm{C}^{\mathcal{M}_{\alpha}}, \mathrm{CI}^{\mathcal{M}_{+\alpha}} \\
\mathrm{C}^{\mathcal{R}_{\alpha}}, \mathrm{CI}^{\mathcal{R}_{+\alpha}} \\
\mathrm{C}^{\mathcal{R}_{\alpha}}, \mathrm{CI}^{\mathcal{R}_{+\alpha}}\end{array}$ & $\begin{array}{l}\mathrm{C}^{\mathcal{M}_{\alpha}}, \mathrm{CI}^{\mathcal{M}_{+\alpha}} \\
\mathrm{C}^{\mathcal{R}_{\alpha}}, \mathrm{CI}^{\mathcal{R}_{+\alpha}} \\
\mathrm{C}^{\mathcal{I}_{\alpha}}, \mathrm{CI}^{\mathcal{I}_{+\alpha}}\end{array}$ \\
\hline
\end{tabular}
assume an $s$-wave superconductor.

\section{CRYSTALLINE-SYMMETRY-BREAKING MASS TERMS}

In this section, we consider model Hamiltonians of a simple, canonical form, which are still sufficiently general that the model description can be applied to all tenfold-way and Shiozaki-Sato classes. We count how many independent "mass terms" can be added to the Hamiltonian that satisfy the fundamental nonspatial (anti)symmetries $\mathcal{T}, \mathcal{P}$, and $\mathcal{C}$ defining the tenfold-way class, but break the crystalline (anti)symmetry $\mathcal{S}$ that determines the Shiozaki-Sato class and show that such mass terms can be used to construct fully $\mathcal{S}$-(anti)symmetric models in which a "boundary mass term" appears on boundaries that are not invariant under the crystalline (anti)symmetry $\mathcal{S}$. This naturally explains the phenomenology of higher-order topological phases in these models. This section serves as the summary of the approach of Refs. [30,41] and as an interlude to the subsequent, more formal section.

Explicitly, the model Hamiltonians we consider have the form

$$
H_{0}(\boldsymbol{k}, m)=\sum_{j=0}^{d} d_{j}(\boldsymbol{k}) \Gamma_{j},
$$

with matrices $\Gamma_{j}$ that anticommute mutually and square to the identity. For the functions $d_{j}$ we choose

$$
\begin{aligned}
d_{0}(\boldsymbol{k}, m) & =m+\sum_{i=1}^{d}\left(1-\cos k_{i}\right), \\
d_{j}(\boldsymbol{k}) & =\sin k_{j} \quad \text { for } j=1, \ldots, d,
\end{aligned}
$$

although our considerations do not change if a different choice for the functions $d_{j}$ is made, as long as the map $\boldsymbol{d} /|\boldsymbol{d}|: T^{d} \rightarrow S^{d}$ has a winding number equal to one for 
$-2<m<0$ and to zero for $0<m<2$, and the vector $\boldsymbol{d}=\left(d_{0}, d_{1}, \ldots, d_{d}\right)$ transforms the same as $(1, \boldsymbol{k})$ under the crystalline (anti)symmetry $\mathcal{S}$ and the nonspatial (anti) symmetries $\mathcal{T}, \mathcal{P}$, and $\mathcal{C}$. The nonspatial (anti)symmetries $\mathcal{T}, \mathcal{P}$, and $\mathcal{C}$ and the crystalline (anti)symmetry $\mathcal{S}$ impose restrictions on the possible choices for the matrices $\Gamma_{j}, j=0,1, \ldots, d$, which we do not specify explicitly here.

We consider the regime $-2<m<0$, for which the Hamiltonian (10) has a band inversion near $\boldsymbol{k}=0$ but not elsewhere in the Brillouin zone. In this parameter range, a Hamiltonian of the form (10) describes a nontrivial topological crystalline phase if there exists no mass term $M$-a Hermitian matrix $M$ squaring to the identity and anticommuting with the Hamiltonian-that satisfies the constraints imposed by $\mathcal{S}$ and by $\mathcal{T}, \mathcal{P}$, and/or $\mathcal{C}$. The topological phase is a "tenfold-way phase"-i.e., it remains nontrivial if the crystalline (anti)symmetry $\mathcal{S}$ is broken-if there exists no mass term $M$ which satisfies the constraints imposed by the nonspatial (anti)symmetries $\mathcal{T}, \mathcal{P}$, and/or $\mathcal{C}$ alone, irrespective of the crystalline (anti)symmetry $\mathcal{S}$. On the other hand, if such a $\mathcal{S}$-breaking mass term exists, the Hamiltonian (10) describes a "purely crystalline" topological phase, which relies on the crystalline (anti)symmetry $\mathcal{S}$ for its protection. Whereas a nontrivial tenfold-way phase is always a first-order phase, the purely crystalline phases can be higher-order topological phases.

In principle, a Hamiltonian of the form (10) may allow for more than one $\mathcal{S}$-breaking mass term-where we require that different $\mathcal{S}$-breaking mass terms $M_{l}$ not only anticommute with $H$, but also with each other. If a canonical-form Hamiltonian has the minimum possible dimension for a given topological class, the $\mathcal{S}$-breaking mass terms $M_{l}$ all change sign under the (anti)symmetry $\mathcal{S}$. In this case, as we argue below, there is a connection between the number of mutually anticommuting $\mathcal{S}$-breaking mass terms and the order $n$ of the topological phase: The presence of $n-1 \mathcal{S}$-breaking mass terms $M_{l}, l=$ $1, \ldots, n-1$ gives rise to a topological phase of order $\min \left(n, d_{\|}+1\right)$ if $\min \left(n, d_{\|}+1\right) \leq d$, and to a boundary without protected in-gap states if $d_{\|}=d$ and $n>d$ [62].

To establish this connection one constructs the lowenergy boundary theory for a Hamiltonian of the form (10) $[20,41]$. This requires considering a Hamiltonian with a slowly position-dependent parameter $m(\mathbf{r})$, such that the topological phase occupies the region for which $m<0$, whereas the region $m>0$ hosts a trivial gapped phase. Whereas the Hamiltonian (10) becomes gapless at the boundary at $m=0$, with the help of $\mathcal{S}$-breaking mass terms $M_{l}$ one may construct a perturbation $H_{1}$ that respects the (anti)symmetry $\mathcal{S}$ and that gaps out the boundary, up to a region of codimension $\max \left(n, d_{\|}+1\right)$,

$$
H_{1}=i \sum_{l=1}^{n-1} \sum_{j=1}^{d_{\|}} b_{j}^{(l)} M_{l} \Gamma_{0} \Gamma_{j},
$$

where, for technical convenience, we take the coefficients $b_{j}^{(l)}$ numerically small. The relation between the number of $\mathcal{S}$-breaking mass terms and the order of the topological phase then follows immediately.

We now verify this statement explicitly for $d=2$. The construction is easily generalized to higher dimensions. Starting from the low-energy limit of the Hamiltonian $H_{0}$ of Eq. (10) with $d=2$ in the vicinity of a boundary with normal $\boldsymbol{n}=\left(n_{1}, n_{2}\right)=(\cos \phi, \sin \phi)$, we find that the projection operator onto low-energy boundary states is [41]

$$
\begin{aligned}
P(\phi) & =\frac{1}{2}\left(i \Gamma_{1} \Gamma_{0} \cos \phi+i \Gamma_{2} \Gamma_{0} \sin \phi+1\right) \\
& =e^{\phi \Gamma_{2} \Gamma_{1} / 2} P(0) e^{-\phi \Gamma_{2} \Gamma_{1} / 2} .
\end{aligned}
$$

Projecting the bulk Hamiltonian $H_{0}+H_{1}$ to the lowenergy boundary states gives

$$
\begin{aligned}
P(\boldsymbol{n}) H P(\boldsymbol{n})= & e^{\phi \Gamma_{2} \Gamma_{1} / 2} P(0) \\
& \times\left(-i \hbar \Gamma_{2} \partial_{x_{b}}+\sum_{l=1}^{n-1} m_{l}(\phi) M_{l}\right) \\
& \times P(0) e^{-\phi \Gamma_{2} \Gamma_{1} / 2},
\end{aligned}
$$

where $m_{l}(\phi)=\sum_{j=1}^{d_{\|}} b_{j}^{(l)} n_{j}$ and $\partial_{x_{b}}=\cos \phi \partial_{x_{2}}-\sin \phi \partial_{x_{1}}$ is the derivative with respect to a coordinate along the edge. We conclude that the effective boundary Hamiltonian reads

$$
H_{\text {boundary }}=-i \hbar \Gamma_{2}^{\prime} \partial_{x_{b}}+\sum_{l=1}^{n-1} m_{l}(\phi) M_{l}^{\prime},
$$

where $\quad \Gamma_{2}^{\prime}=P(0) \Gamma_{2} P(0) \quad$ and $\quad M_{l}^{\prime}=P(0) M_{l} P(0)$. Alternatively, one may arrive at the effective boundary Hamiltonian (15) by starting from the canonical-form Hamiltonian (10) and adding the perturbation $M_{l}$ locally at the boundary, provided the boundary is not itself invariant under $\mathcal{S}$ and the prefactor $m_{l}(\phi)$ obeys the restrictions imposed by $\mathcal{S}$ [as it does in Eq. (15)].

The boundary Hamiltonian (15) hosts zero-energy corner states between crystal edges with opposite sign of $m_{l}(\phi)$, provided all mass terms $m_{l}(\phi)$ go through zero at the same value of $\phi$. For an on-site order-two symmetry $\mathcal{O}$ with $d_{\|}=0$, the mass terms $M_{l}$ cannot be used to construct an $\mathcal{O}$-preserving perturbation, which is consistent with the absence of $\mathcal{O}$-symmetry-breaking boundaries. Mirror symmetry has $d_{\|}=1$ flipped coordinates, which gives $m_{l}(\phi)=b_{1}^{(l)} \cos \phi$ : all mass terms $m_{l}(\phi)$ vanish simultaneously on the mirror line and one obtains a second-order phase whenever there is at least one mass term, i.e., if $n \geq 2$. Finally, a twofold rotation symmetry has $d_{\|}=2$, and zero-energy corner states are obtained only if the number $n-1$ of crystalline-symmetry-breaking terms is 
exactly one. For $n>2$, the coefficients $b_{j}^{(l)}$ can be chosen to yield a fully gapped boundary, which describes the situation where the bulk is topologically nontrivial but the boundary does not host any anomalous states-in this case the group $K^{(d)}$ is nontrivial.

\section{BULK AND BOUNDARY CLASSIFICATION OF TOPOLOGICAL CRYSTALLINE INSULATORS}

We now turn to a general topological classification of the electronic structure of a $d$-dimensional crystal with an order-two crystalline symmetry or antisymmetry $\mathcal{S}$ with $d_{\|}$ inverted dimensions. We assume that the crystal shape, including the lattice termination, is compatible with the crystalline symmetry. We recall that the system is in an $n$th order topological phase if it has protected boundary states of codimension $n$, whereas the bulk and all boundaries of codimension smaller than $n$ are gapped. In this section, we establish the formal framework for a classification of such $n$ th-order topological phases, both from a bulk perspective and from a boundary perspective, and show the extent to which they are related.

As announced in the Introduction of this article, the bulkperspective classification amounts to the construction of the subgroup series (1) of classifying groups $K^{(n)}$, where $K^{(n)}$ classifies the topology of bulk band structures excluding topological phases of order $\leq n$. Since taking the direct sum of two topological phases cannot reduce the codimension of anomalous boundary states, the $K^{(n)}$ defined this way have a well-defined group structure in the Grothendieck construction. Figure 2 illustrates the definitions of the subgroup sequence for the case of a three-dimensional crystal with twofold rotation symmetry.

The definition of the boundary-perspective classification groups requires a little more care, because for boundary states of order $n>1$ their location, number, and type may depend on the crystal shape and crystal termination. A classifying group that is independent of such details is obtained by considering equivalence classes of configurations of codimension- $n$ boundary states that differ by a change in lattice termination only. This is the classifying group $\mathcal{K}_{a}^{(n)}$ of anomalous boundary states that appears in the bulk-boundary correspondence (2). In this section, we pursue a further resolution of the boundary classification, by defining boundary classification groups $\mathcal{K}_{k}^{(n)}$ of equivalence classes of codimension- $n$ boundary states that differ by the lattice termination along boundaries of codimension $\geq k$ only, $k=1, \ldots, n-1$. With that definition, the classifying group of anomalous boundary states

$$
\mathcal{K}_{a}^{(n)}=\mathcal{K}_{1}^{(n)}
$$

Specifically, for a three-dimensional crystal, $\mathcal{K}_{a}^{\prime \prime}=\mathcal{K}_{1}^{\prime \prime}$ classifies configurations of protected gapless modes along hinges, where configurations that differ by termination only are identified. Similarly, $\mathcal{K}_{a}^{\prime \prime \prime}=\mathcal{K}_{1}^{\prime \prime \prime}$ classifies configurations of protected zero-energy states at crystal corners, again identifying configurations of corner states that differ by a change of lattice termination. The group $\mathcal{K}_{2}^{\prime \prime \prime}$ classifies configurations of protected zero-energy corner states, identifying configurations that differ by changing the termination along crystal hinges, without affecting the crystal faces.

Location of boundary states for $n>d_{\|}$. - A crystalline symmetry $\mathcal{S}$ with $d_{\|}$inverted dimensions necessarily leaves a manifold $\Omega_{d-d_{\|}}$invariant. For $d_{\|}=1$ this is the mirror plane; for $d_{\|}=2$ it is the twofold rotation axis; see Fig. 3. For boundary states of codimension $n>d_{\|}$it is always possible to change the crystal termination along boundaries of codimension $n-1$ only, such that all boundary states end up on the intersection $\partial \Omega_{d-d_{\|}}$of the invariant manifold $\Omega_{d-d_{\|}}$and the crystal boundary. Examples of such a procedure are shown schematically in Fig. 4 for a twodimensional crystal with mirror symmetry and for a threedimensional crystal with twofold rotation symmetry. We conclude, that for $n>d_{\|}$it is sufficient to consider configurations of codimension- $n$ boundary states with support on $\partial \Omega_{d-d_{\|}}$only.

Classifying groups for $n>d_{\|}+1$.-We now combine this conclusion with the observation that the crystalline symmetry $\mathcal{S}$ is a local (i.e., on-site) symmetry inside the invariant manifold $\Omega_{d-d_{\|}}$. The "conventional" tenfold-way bulk-boundary correspondence, according to which any anomalous states are the first-order boundary phase of a topological phase, remains valid in the presence of a local crystalline symmetry. Applying this bulk-boundary correspondence to protected gapless boundary states of codimension $n>d_{\|}+1$ within $\partial \Omega_{d-d_{\|}}$, such states can be (a)

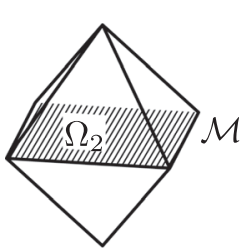

(c)

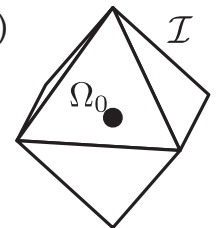

(b)
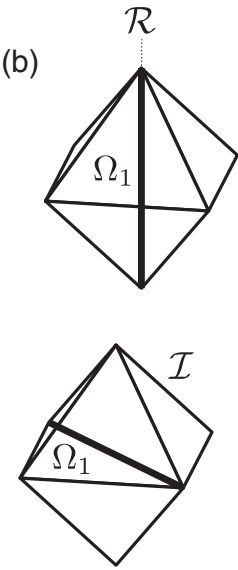
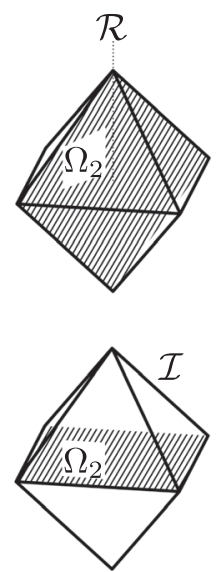

FIG. 3. Manifolds $\Omega_{k}$ with $d-d_{\|} \leq k<d$ for threedimensional crystals with mirror (a), twofold rotation (b), and inversion (c) symmetry. The manifold $\Omega_{d}$ is equal to the entire crystal, see Eq. (19), and is not shown in the figure. 
(a)

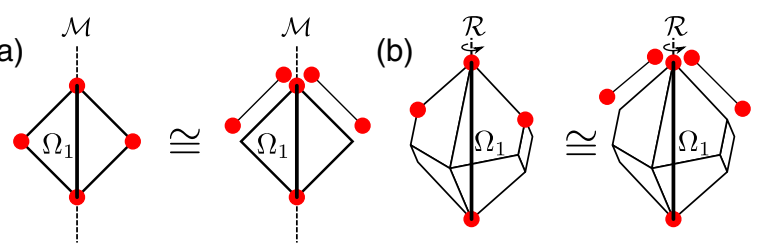

(c)

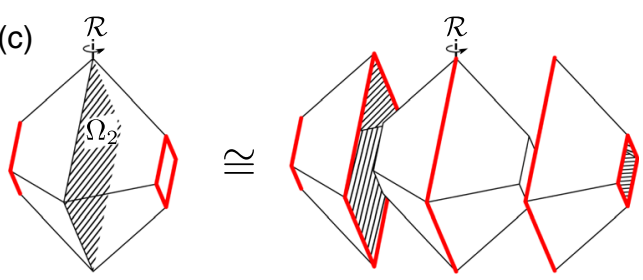

FIG. 4. By attaching a "decoration" consisting of a first-order topological phase on a boundary of codimension $n-1$, an arbitrary configuration of boundary state of codimension $n$ can be moved to the subset $\partial \Omega_{d+1-n}$. The figure shows three examples: Corner states of a two-dimensional crystal with mirror symmetry $(n=2)$, which can always be moved to the intersection of the mirror line and the crystal boundary upon changing the crystal termination (a), corner states of a three-dimensional crystal with twofold rotation symmetry $(n=3)$, which can always be moved to the intersection points of the twofold rotation axis and the crystal boundary upon changing the termination along crystal hinges (b), and hinge states of a three-dimensional crystal with twofold rotation symmetry, which can always be moved to the intersection of the two-dimensional manifold $\Omega_{2}$ and the crystal boundary upon changing the termination at crystal faces (c).

interpreted as the first-order boundary states of a codimension- $(n-1)$ topological phase, still located within the invariant part $\partial \Omega_{d-d_{\|}}$of the crystal boundary. Obviously, such boundary states can be removed by changing crystal termination along boundaries of codimension $n-1$. It follows that the boundary classification groups $\mathcal{K}_{k}^{(n)}$ are all trivial,

$$
\mathcal{K}_{k}^{(n)}=0, \text { for } n>d_{\|}+1 \text { and } k=1, \ldots, n-1 .
$$

A similar argument can be made for the bulk classification groups $K^{(n)}$ for $n>d_{\|}+1$. Again, because $\mathcal{S}$ is a local symmetry on $\partial \Omega_{d-d_{\|}}$, a nontrivial bulk topology implies the presence of protected gapless boundary states of codimension $d_{\|}+1$ or less (see, e.g., Sec. III and Refs. [41,48]). Equation (3) follows immediately from this observation, which, combined with the relation (17), yields the bulkboundary correspondence (2) highlighted in the introduction for $n>d_{\|}$.

Boundary classification for $n=d_{\|}+1$. - The calculation the groups $\mathcal{K}_{k}^{(n)}$ for $n=d_{\|}+1$ proceeds via a series of auxiliary groups $\mathcal{D}_{k}^{(n)}$. The first of these, $\mathcal{D}_{0}^{(n)}$, is defined as the classifying group of codimension- $n$ boundary states with support entirely within $\partial \Omega_{d-d_{\|}}$. We refer to this group as the "extrinsic boundary classification group." To relate $\mathcal{D}_{0}^{(n)}$ to the known classification groups of first-order topological phases, we argue that (i) the $\left(d-d_{\|}-1\right)$ dimensional boundary states on $\partial \Omega_{d-d_{\|}}$may be interpreted as first-order boundary states of $\Omega_{d-d_{\|}}$and (ii) $\mathcal{S}$ is a local symmetry on $\Omega_{d-d_{\|}}$. For (i) it is essential that the crystal boundary is fully gapped away from $\partial \Omega_{d-d_{\|}}$, so that the crystal away from $\Omega_{d-d_{\|}}$may be considered effectively topologically trivial and one may consider the manifold $\Omega_{d-d_{\|}}$in isolation. This immediately gives the identification

$$
\mathcal{D}_{0}^{(n)}=K\left(d-d_{\|}, 0\right),
$$

where $K\left(d-d_{\|}, 0\right)$ is the Shiozaki-Sato classifying group for a $\left(d-d_{\|}\right)$-dimensional crystal with an on-site crystalline symmetry. Before we proceed with the definition of the remaining groups $\mathcal{D}_{k}^{(n)}$ and the construction of the boundary classification groups $\mathcal{K}_{k}^{(n)}$, we first discuss how the above construction is generalized to boundary states of codimension $n \leq d_{\|}$.

Boundary classification for $n \leq d_{\|}$.- To calculate the boundary classifying groups $\mathcal{K}_{k}^{(n)}$ for $n \leq d_{\|}$, we define a sequence of manifolds

$$
\Omega_{d-d_{\|}} \subset \Omega_{d-d_{\|}+1} \subset \ldots \subset \Omega_{d},
$$

where $\Omega_{d}$ equals the entire crystal, whereas the $k$-dimensional manifolds $\Omega_{k}$ for $d-d_{\|} \leq k<d$ are mapped into themselves under the crystalline symmetry $\mathcal{S}$. We additionally require that the intersection $\partial \Omega_{d+1-n}$ with the crystal boundary is along crystal boundaries of codimension $n$. Examples of such a sequence of manifolds $\Omega_{k}$ are shown in Figs. 3(b) and 3(c). With this construction, one easily verifies that by changing crystal termination along boundaries of codimension $n-1$ only, any configuration of codimension- $n$ boundary states can be made to lie entirely within $\partial \Omega_{d+1-n}$.

Generalizing the above discussion for the case $n=d_{\|}+1$, we define the extrinsic classifying group $\mathcal{D}_{0}^{(n)}$ as the classifying group of codimension- $n$ boundary states with support entirely within $\partial \Omega_{d+1-n}$. To relate $\mathcal{D}_{0}^{(n)}$ to the known classification groups of first-order topological phases, we again interpret boundary states on $\partial \Omega_{d+1-n}$ as first-order boundary states of $\Omega_{d+1-n}$. A difference with the case $n=d_{\|}+1$ is that now the order-two crystalline (anti) symmetry $\mathcal{S}$ is a nonlocal symmetry with $d_{\|}+1-n$ inverted dimensions. We thus find

$$
\mathcal{D}_{0}^{(n)}=K_{\mathrm{TF}, \mathcal{S}}\left(d+1-n, d_{\|}+1-n\right),
$$

where $K_{\mathrm{TF}, \mathcal{S}} \subseteq K_{\mathrm{TF}}$ classifies the tenfold-way phases compatible with the nonlocal crystalline symmetry $\mathcal{S}$; see Sec. II. 
The boundary classification groups $\mathcal{K}_{k}^{(n)}$ describe equivalence classes of configurations of codimension- $n$ boundary states with respect to changes of the lattice termination for boundaries of codimension $\geq k$. In other words, when seen as an element of $\mathcal{K}_{k}^{(n)}$, a codimension- $n$ state on $\partial \Omega_{d+1-n}$ is considered trivial if it can be obtained as the boundary state of a $(d-k)$-dimensional $(n-k)$ th order topological phase entirely contained within the crystal boundary and respecting the global (anti)symmetry $\mathcal{S}$. Denoting the classifying group for such "trivial" boundary states as $\mathcal{D}_{k}^{(n)}$, the boundary classification group $\mathcal{K}_{k}^{(n)}$ can thus be obtained as the quotient

$$
\mathcal{K}_{k}^{(n)}=\mathcal{D}_{0}^{(n)} / \mathcal{D}_{k}^{(n)}, \quad k=2, \ldots, n,
$$

where $\mathcal{D}_{0}^{(n)}$ is the extrinsic boundary classifying group introduced above. The groups $\mathcal{D}_{k}^{(n)}$, which we call "decoration groups," form a subgroup sequence that also includes the extrinsic boundary classification group,

$$
\mathcal{D}_{n-1}^{(n)} \subseteq \cdots \subseteq \mathcal{D}_{1}^{(n)} \subseteq \mathcal{D}_{0}^{(n)} .
$$

Figure 5 shows examples of this subgroup sequence for a two-dimensional crystal with mirror symmetry and a threedimensional crystal with twofold rotation symmetry. Since the crystalline symmetry $\mathcal{S}$ acts nonlocally for a generic position in a $(d-k)$-dimensional boundary state, the Hamiltonian of such a decoration state is "separable," i.e., it may be written as

$$
\left(\begin{array}{cc}
h_{d-k}(\boldsymbol{k}) & 0 \\
0 & \tilde{\mathcal{S}} h_{d-k}(\boldsymbol{k})
\end{array}\right),
$$

where $h_{d-k}$ is a $(d-k)$-dimensional Hamiltonian without crystalline symmetries and

$$
\mathcal{S}=\left(\begin{array}{ll}
0 & 1 \\
1 & 0
\end{array}\right) \tilde{\mathcal{S}}
$$

where $\tilde{\mathcal{S}}$ is an (anti)symmetry operator with $d_{\|}-k$ inverted dimensions acting on $(d-k)$-dimensional Hamiltonians. [Note that the boundary of a decoration need not be a separable in this sense. This is illustrated schematically in Fig. 5(b). Further examples are given in Sec. VI.]

Bulk-boundary correspondence for $n \leq d_{\|}$.-To establish a relation between the bulk classifying groups $K^{(n)}$ and the decoration subgroups $\mathcal{D}_{k}^{(n)}$ for $n \leq d_{\|}$we make use of a homomorphism

$$
K\left(d, d_{\|}\right) \stackrel{\omega}{\rightarrow} K\left(d+1, d_{\|}+1\right)
$$

which maps an equivalence class of $d$-dimensional Hamiltonians $H$ in Shiozaki-Sato class $\left(s, t, d_{\|}\right)$to a

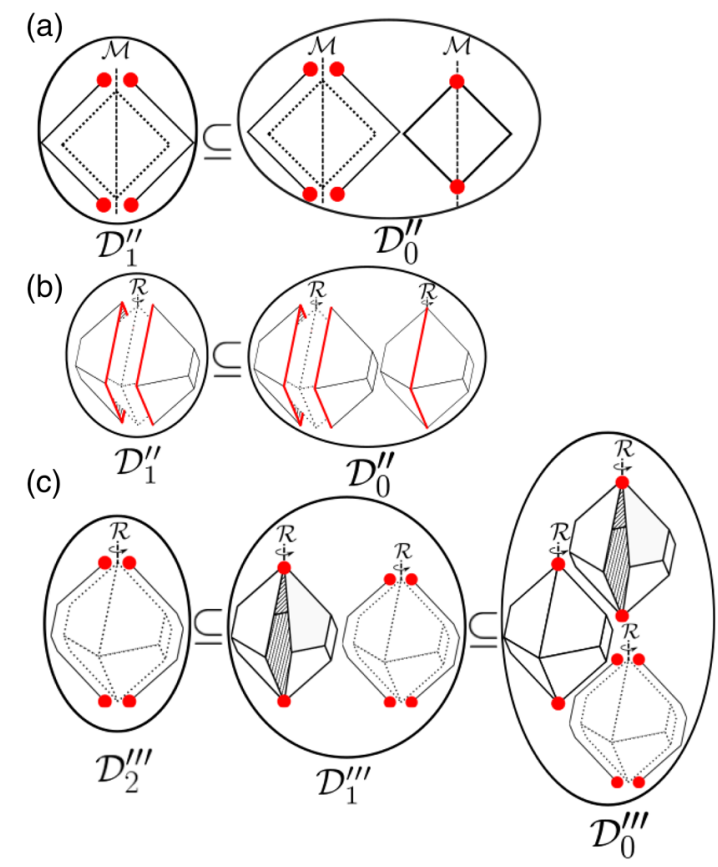

FIG. 5. Examples of the subgroup sequence (22) for the decoration subgroups $\mathcal{D}_{k}^{(n)}$ and the extrinsic boundary classifying group $\mathcal{D}_{0}^{(n)}$. (a) For a two-dimensional crystal with mirror symmetry, the group $\mathcal{D}_{0}^{\prime \prime}$ classifies all possible configurations of corner states on the mirror axis, whereas the decoration subgroup $\mathcal{D}_{1}^{\prime \prime}$ classifies corner states on the mirror axis that can be obtained by "gluing" two mirror-related first-order topological phases to the crystal boundary. (b) For a threedimensional crystal with twofold rotation symmetry, $\mathcal{D}_{0}^{\prime \prime}$ classifies all configurations of protected hinge modes along the intersection $\partial \Omega_{2}$ of the reference manifold $\Omega_{2}$ and the crystal boundary, whereas the decoration subgroup $\mathcal{D}_{1}^{\prime \prime}$ classifies all protected hinge modes along $\partial \Omega_{2}$ that are obtained by gluing two rotation-related first-order topological phases to the crystal boundary. (c) For a three-dimensional crystal with twofold rotation symmetry, $\mathcal{D}_{0}^{\prime \prime \prime}$ classifies all configurations of protected zero-energy states at the two corners on the rotation axis. The subgroups $\mathcal{D}_{2}^{\prime \prime \prime}$ and $\mathcal{D}_{1}^{\prime \prime \prime}$ contain corner states on the rotation axis that are obtained from decorating rotation-related hinges or surfaces with first-order and second-order topological phases, respectively.

$(d+1)$-dimensional Hamiltonian in Shiozaki-Sato class $\left(s, t, d_{\|}+1\right)$. The precise definition of the homomorphism will be given in Sec. VI. For the derivation of the bulkboundary correspondence (2), it will be sufficient to use three key properties of $\omega$ :

(i) $\omega(H)$ is in the trivial class if and only if $H$ is separable if $H$ can be deformed to a separable Hamiltonian.

(ii) The homomorphism $\omega$ commutes with the dimensionraising isomorphisms $\kappa_{\|}$and $\kappa_{\perp}$, up to a possible sign change of the topological invariants.

(iii) If $H$ is a nonseparable Hamiltonian with $n-1$ crystalline-symmetry-breaking mass terms, then $\omega(H)$ is a Hamiltonian with $n$ crystalline-symmetry-breaking 
mass terms. The inverse is also true: $H \in \operatorname{img} \omega$ if $H$ has at least one $\mathcal{S}$-breaking mass term.

The third property ensures that $\omega$ does not change the dimension of the protected boundary states (if any). For that reason, we refer to $\omega$ as the order-raising homomorphism, as it increases the order of the topological phase by one. A proof of these three properties will be given in Appendix $\mathrm{C}$ for the homomorphism that we will introduce in Sec. VI. The stacking construction previously considered in the literature $[53,54,63]$ is another realization of the order-raising homomorphism-this is explicitly demonstrated in Sec. VI C.

The proof of the bulk-boundary correspondence (2) makes use of the three properties of $\omega$, without requiring knowledge of the specific form of the homomorphism. Here, too, we first note that the last of these properties can be used to calculate the bulk classifying groups $K^{(n)}$ in the subgroup series (1), since the number $n-1$ of crystallinesymmetry-breaking mass terms is related to the order $n$ of the topological phase (provided $n \leq d-1$ ); see Sec. III and Refs. [30,41]. We conclude that Hamiltonians in $K^{(n)}$ must have at least $n$ mass terms on a boundary if $n \leq d_{\|}$, so that

$$
K^{(n)}\left(d, d_{\|}\right)=\omega^{n}\left[K\left(d-n, d_{\|}-n\right)\right] .
$$

In particular, the purely crystalline subgroup $K^{\prime}\left(d, d_{\|}\right)$ consists of the (classes of) Hamiltonians with at least one mass term on the boundary,

$$
K^{\prime}\left(d, d_{\|}\right)=\omega\left[K\left(d-1, d_{\|}-1\right)\right] .
$$

Similarly, the first property of the order-raising homomorphism $\omega$ leads to an expression for the decoration subgroups. We first consider the case $n=d_{\|}+1$, for which one has $\mathcal{D}_{0}^{(n)}=K(d+1-n, 0)$; see Eq. (20). In this case, we find that the decoration subgroups $\mathcal{D}_{k}^{(n)} \subseteq$ $\mathcal{D}_{0}^{(n)}$ are given by

$$
\mathcal{D}_{k}^{(n)}=\operatorname{ker} \omega^{n-k},
$$

since $\mathcal{D}_{k}^{(n)}$ classifies codimension- $n$ boundary states from separable $(k-1)$ th order Hamiltonians. For the classifying group $\mathcal{K}_{k}^{(n)}$ this gives

$$
\mathcal{K}_{k}^{(n)}=K\left(d+1-n, d_{\|}+1-n\right) / \operatorname{ker} \omega^{n-k}
$$

if $n=d_{\|}+1$. For $n<d_{\|}+1$ one finds similarly, using the isomorphism (9),

$$
\begin{aligned}
\mathcal{D}_{k}^{(n)}= & K^{\prime}\left(d+1-n, d_{\|}+1-n\right) \operatorname{ker} \omega^{n-k} \\
& / K^{\prime}\left(d+1-n, d_{\|}+1-n\right),
\end{aligned}
$$

where the subgroup $K^{\prime}\left(d+1-n, d_{\|}+1-n\right) \operatorname{ker} \omega^{n-k} \subseteq$ $K\left(d+1-n, d_{\|}+1-n\right)$ consists of direct sums $g \oplus h$, with $g \in K^{\prime}\left(d+1-n, d_{\|}+1-n\right)$ and $h \in \operatorname{ker} \omega^{n-k}$. (Note that all classifying groups considered here are Abelian.) This gives the compact expression

$$
\begin{aligned}
\mathcal{K}_{k}^{(n)}= & K\left(d+1-n, d_{\|}+1-n\right) \\
& / K^{\prime}\left(d+1-n, d_{\|}+1-n\right) \operatorname{ker} \omega^{n-k} .
\end{aligned}
$$

Note that Eq. (28) can be considered a special case of Eq. (30) since $K^{\prime}\left(d+1-n, d_{\|}+1-n\right)$ is trivial if $n=d_{\|}+1$. The bulk-boundary correspondence (2) now follows from Eqs. (30) with $k=1$ and Eqs. (26) and (25) upon applying the general group isomorphism $K / G \operatorname{ker} \alpha=\alpha[K] / \alpha[G]$ for any subgroup $G \subseteq K$ and homomorphism $\alpha$ to the case $K=K(d+1-n$, $\left.d_{\|}+1-n\right), G=\omega\left[K\left(d-n, d_{\|}-n\right)\right]$, and $\alpha=\omega^{n}$ [64]. In Appendix A we discuss a possible way to extend the above proof to an arbitrary crystalline symmetry.

Calculation of the subgroup sequence.-The bulk classifying groups $K\left(d, d_{\|}\right)$were calculated by Shiozaki and Sato in Ref. [21]. The purely crystalline subgroups $K^{\prime}\left(d, d_{\|}\right)$were calculated in Refs. [30,41] by explicit calculation for each Shiozaki-Sato symmetry class separately. (Although Ref. [41] considered $d_{\|} \geq 1$ for $d=2$ and $d=3$ only, the results can be transferred to all other Shiozaki-Sato classes using the dimension-raising and lowering isomorphisms $\kappa_{\|}$and $\kappa_{\perp}$.) As shown in Appendix D, the kernels ker $\omega \subseteq K\left(d, d_{\|}\right)$can be obtained from the known results for $K^{\prime}$ and $K$.

The remainder of the calculation of the classifying groups can be done without further explicit calculations. This relies on the key observation that the nontrivial groups in the sequence

$$
\begin{aligned}
K^{\prime}\left(d+1, d_{\|}+1\right) & \rightarrow K^{\prime}\left(d+2, d_{\|}+2\right) \\
& \rightarrow K^{\prime}\left(d+3, d_{\|}+3\right) \rightarrow \ldots
\end{aligned}
$$

TABLE X. Boundary classification of third-order phases in three-dimensional systems with an order-two symmetry (antisymmetry) for complex tenfold-way classes. The symbols $\mathcal{O}, \mathcal{M}$, $\mathcal{R}$, and $\mathcal{I}$ refer to a local on-site $\left(d_{\|}=0\right)$, mirror $\left(d_{\|}=1\right)$, twofold rotation $\left(d_{\|}=2\right)$, and inversion symmetry $\left(d_{\|}=3\right)$, respectively. The boundary classification groups are given in the order $\mathcal{D}_{0}^{\prime \prime \prime}, \mathcal{K}_{2}^{\prime \prime \prime}, \mathcal{K}_{1}^{\prime \prime \prime}=\mathcal{K}_{a}^{\prime \prime \prime}$.

\begin{tabular}{lcccccc}
\hline \hline TF class & $s$ & $t$ & $\mathcal{O}$ & $\mathcal{M}$ & $\mathcal{R}$ & $\mathcal{I}$ \\
\hline $\mathrm{A}^{\mathcal{S}}$ & 0 & 0 & $0,0,0$ & $0,0,0$ & $0,0,0$ & $0,0,0$ \\
$\mathrm{AIII}^{\mathcal{S}_{+}}$ & 1 & 0 & $\mathbb{Z}^{2}, 0,0$ & $\mathbb{Z}^{2}, 0,0$ & $\mathbb{Z}^{2}, \mathbb{Z}, \mathbb{Z}$ & $0,0,0$ \\
\hline $\mathrm{A}^{\mathcal{C S}}$ & 0 & 1 & $\mathbb{Z}, 0,0$ & $\mathbb{Z}, 0,0$ & $\mathbb{Z}, \mathbb{Z}, \mathbb{Z}$ & $0,0,0$ \\
$\mathrm{AIII}^{\mathcal{S}_{-}}$ & 1 & 1 & $0,0,0$ & $0,0,0$ & $0,0,0$ & $\mathbb{Z}, \mathbb{Z}_{2}, \mathbb{Z}_{2}$ \\
\hline \hline
\end{tabular}


TABLE XI. Same as Table X, but for antiunitary symmetries and antisymmetries.

\begin{tabular}{lccccc}
\hline \hline $\begin{array}{l}\text { Shiozaki-Sato } \\
\text { class }\end{array}$ & $s$ & $\mathcal{O}$ & $\mathcal{M}$ & $\mathcal{R}$ & $\mathcal{I}$ \\
\hline $\mathrm{A}^{\mathcal{T}^{+} \mathcal{S}}$ & 0 & $0,0,0$ & $0,0,0$ & $0,0,0$ & $0,0,0$ \\
$\mathrm{AIII}^{\mathcal{P}^{+} \mathcal{S}_{+}}$ & 1 & $\mathbb{Z}, 0,0$ & $\mathbb{Z}, 0,0$ & $\mathbb{Z}, \mathbb{Z}_{2}, \mathbb{Z}_{2}$ & $0,0,0$ \\
$\mathrm{~A}^{\mathcal{P}^{+} \mathcal{S}}$ & 2 & $\mathbb{Z}_{2}, 0,0$ & $\mathbb{Z}_{2}, 0,0$ & $\mathbb{Z}_{2}, \mathbb{Z}_{2}, 0$ & $0,0,0$ \\
$\mathrm{AIII}^{\mathcal{T}^{-}} \mathcal{S}_{-}$ & 3 & $\mathbb{Z}_{2}, 0,0$ & $\mathbb{Z}_{2}, 0,0$ & $\mathbb{Z}_{2}, 0,0$ & $2 \mathbb{Z}, 0,0$ \\
$\mathrm{~A}^{\mathcal{T}^{-} \mathcal{S}}$ & 4 & $0,0,0$ & $0,0,0$ & $0,0,0$ & $0,0,0$ \\
$\mathrm{AIII}^{\mathcal{P}^{-} \mathcal{S}_{+}}$ & 5 & $2 \mathbb{Z}, 0,0$ & $2 \mathbb{Z}, 0,0$ & $2 \mathbb{Z}, 0,0$ & $0,0,0$ \\
$\mathrm{~A}^{\mathcal{P}^{-} \mathcal{S}}$ & 6 & $0,0,0$ & $0,0,0$ & $0,0,0$ & $0,0,0$ \\
$\mathrm{AIII}^{\mathcal{T}^{+} \mathcal{S}_{-}}$ & 7 & $0,0,0$ & $0,0,0$ & $0,0,0$ & $\mathbb{Z}, \mathbb{Z}_{2}, \mathbb{Z}_{2}$ \\
\hline \hline
\end{tabular}

are isomorphic to $\mathbb{Z}$ or to $\mathbb{Z}_{2}$ and that the succession $\mathbb{Z} \rightarrow$ $\mathbb{Z}_{2}$ does not occur. Since both $\mathbb{Z}$ and $\mathbb{Z}_{2}$ have a single generator, it follows that any homomorphism $K^{\prime}(d+l$, $\left.d_{\|}+l\right) \rightarrow K^{\prime}\left(d+l+1, d_{\|}+l+1\right)$ is either injective, or it maps $K^{\prime}\left(d+l, d_{\|}+l\right)$ to the trivial element. Applying this observation to the order-raising homomorphism $\omega$ and denoting the first instance in which $\omega$ maps $K^{\prime}(d+l$, $\left.d_{\|}+l\right)$ to the trivial element by $K^{\prime}\left(d+q, d_{\|}+q\right)$, we obtain the sequence

$$
\begin{aligned}
K^{\prime}\left(d+1, d_{\|}+1\right) & \stackrel{\omega}{\hookrightarrow} K^{\prime}\left(d+2, d_{\|}+2\right) \stackrel{\omega}{\hookrightarrow} \ldots \\
& \stackrel{\omega}{\hookrightarrow} K^{\prime}\left(d+q, d_{\|}+q\right) \stackrel{\omega}{\rightarrow} 0,
\end{aligned}
$$

TABLE XII. Boundary classification of third-order phases in three-dimensional systems with an order-two symmetry (antisymmetry) for real tenfold-way classes. The symbols $\mathcal{O}, \mathcal{M}, \mathcal{R}$, and $\mathcal{I}$ refer to a local on-site $\left(d_{\|}=0\right)$, mirror $\left(d_{\|}=1\right)$, twofold rotation $\left(d_{\|}=2\right)$, and inversion symmetry $\left(d_{\|}=3\right)$, respectively. The boundary

\begin{tabular}{|c|c|c|c|c|c|c|}
\hline Class & $s$ & $t$ & $\mathcal{O}$ & $\mathcal{M}$ & $\mathcal{R}$ & $\mathcal{I}$ \\
\hline$\overline{\mathrm{AI}^{\mathcal{S}_{+}}}$ & 0 & 0 & $0,0,0$ & $0,0,0$ & $0,0,0$ & $0,0,0$ \\
\hline $\mathrm{BDI}^{\mathcal{S}_{++}}$ & 1 & 0 & $\mathbb{Z}^{2}, 0,0$ & $\mathbb{Z}^{2}, 0,0$ & $\mathbb{Z}^{2}, \mathbb{Z}, \mathbb{Z}$ & $0,0,0$ \\
\hline $\mathrm{D}^{\mathcal{S}_{+}}$ & 2 & 0 & $\mathbb{Z}_{2}^{2}, 0,0$ & $\mathbb{Z}_{2}^{2}, 0,0$ & $\mathbb{Z}_{2}^{2}, \mathbb{Z}_{2}, 0$ & $0,0,0$ \\
\hline $\mathrm{DIII}^{\mathcal{S}_{++}}$ & 3 & 0 & $\mathbb{Z}_{2}^{2}, 0,0$ & $\mathbb{Z}_{2}^{2}, 0,0$ & $\mathbb{Z}_{2}^{2}, \mathbb{Z}_{2}, 0$ & $0,0,0$ \\
\hline $\mathrm{AII}^{\mathcal{S}_{+}}$ & 4 & 0 & $0,0,0$ & $0,0,0$ & $0,0,0$ & $0,0,0$ \\
\hline $\mathrm{CII}^{\mathcal{S}_{++}}$ & 5 & 0 & $2 \mathbb{Z}^{2}, 0$ & $2 \mathbb{Z}^{2}, 0$ & $2 \mathbb{Z}^{2}, 2 \mathbb{Z}, 2 \mathbb{Z}$ & $0,0,0$ \\
\hline $\mathrm{C}^{\mathcal{S}_{+}}$ & 6 & 0 & $0,0,0$ & $0,0,0$ & $0,0,0$ & $0,0,0$ \\
\hline $\mathrm{CI}^{\mathcal{S}_{++}}$ & 7 & 0 & $0,0,0$ & $0,0,0$ & $0,0,0$ & $0,0,0$ \\
\hline $\mathrm{AI}^{\mathcal{C} \mathcal{S}_{-}}$ & 0 & 1 & $0,0,0$ & $0,0,0$ & $0,0,0$ & $0,0,0$ \\
\hline $\mathrm{BDI}^{\mathcal{S}_{+-}}$ & 1 & 1 & $0,0,0$ & $0,0,0$ & $0,0,0$ & $\mathbb{Z}, \mathbb{Z}_{2}, \mathbb{Z}_{2}$ \\
\hline $\mathrm{D}^{\mathcal{C S} \mathcal{S}_{+}}$ & 2 & 1 & $\mathbb{Z}, 0,0$ & $\mathbb{Z}, 0,0$ & $\mathbb{Z}, \mathbb{Z}, \mathbb{Z}$ & $\mathbb{Z}_{2}, \mathbb{Z}_{2}, 0$ \\
\hline $\mathrm{DIII}^{\mathcal{S}_{-+}}$ & 3 & 1 & $\mathbb{Z}_{2}, 0,0$ & $\mathbb{Z}_{2}, 0,0$ & $\mathbb{Z}_{2}, \mathbb{Z}_{2}, 0$ & $\mathbb{Z}_{2}, \mathbb{Z}_{2}, 0$ \\
\hline $\mathrm{AII}^{\mathcal{C} \mathcal{S}_{-}}$ & 4 & 1 & $\mathbb{Z}_{2}, 0,0$ & $\mathbb{Z}_{2}, 0,0$ & $\mathbb{Z}_{2}, \mathbb{Z}_{2}, 0$ & $0,0,0$ \\
\hline $\mathrm{CII}^{\mathcal{S}_{+-}}$ & 5 & 1 & $0,0,0$ & $0,0,0$ & $0,0,0$ & $2 \mathbb{Z}, \mathbb{Z}_{2}, \mathbb{Z}_{2}$ \\
\hline $\mathrm{C}^{\mathcal{C} \mathcal{S}_{+}}$ & 6 & 1 & $2 \mathbb{Z}, 0,0$ & $2 \mathbb{Z}, 0,0$ & $2 \mathbb{Z}, 2 \mathbb{Z}, 2 \mathbb{Z}$ & $0,0,0$ \\
\hline$\underline{\mathrm{CI}^{\mathcal{S}_{-+}}}$ & 7 & 1 & $0,0,0$ & $0,0,0$ & $0,0,0$ & $0,0,0$ \\
\hline$\overline{\mathrm{AI}^{\mathcal{S}_{-}}}$ & 0 & 2 & $0,0,0$ & $0,0,0$ & $0,0,0$ & $0,0,0$ \\
\hline $\mathrm{BDI}^{\mathcal{S}_{--}}$ & 1 & 2 & $2 \mathbb{Z}, 0,0$ & $2 \mathbb{Z}, 0,0$ & $2 \mathbb{Z}, 0,0$ & $0,0,0$ \\
\hline $\mathrm{D}^{\mathcal{S}_{-}}$ & 2 & 2 & $0,0,0$ & $0,0,0$ & $0,0,0$ & $\mathbb{Z}_{2}, \mathbb{Z}_{2}, \mathbb{Z}_{2}$ \\
\hline DIII $^{\mathcal{S}_{--}}$ & 3 & 2 & $2 \mathbb{Z}, 0,0$ & $2 \mathbb{Z}, 0,0$ & $2 \mathbb{Z}, 2 \mathbb{Z}, 2 \mathbb{Z}$ & $\mathbb{Z}_{2}, \mathbb{Z}_{2}, 0$ \\
\hline $\mathrm{AII}^{\mathcal{S}_{-}}$ & 4 & 2 & $0,0,0$ & $0,0,0$ & $0,0,0$ & $0,0,0$ \\
\hline $\mathrm{CII}^{\mathcal{S}_{--}}$ & 5 & 2 & $2 \mathbb{Z}, 0,0$ & $2 \mathbb{Z}, 0,0$ & $2 \mathbb{Z}, \mathbb{Z}_{2}, \mathbb{Z}_{2}$ & $0,0,0$ \\
\hline $\mathrm{C}^{\mathcal{S}_{-}}$ & 6 & 2 & $0,0,0$ & $0,0,0$ & $0,0,0$ & $0,0,0$ \\
\hline $\mathrm{CI}^{\mathcal{S}_{--}}$ & 7 & 2 & $2 \mathbb{Z}, 0,0$ & $2 \mathbb{Z}, 0,0$ & $2 \mathbb{Z}, 2 \mathbb{Z}, 2 \mathbb{Z}$ & $0,0,0$ \\
\hline $\mathrm{AI}^{\mathcal{C} \mathcal{S}_{+}}$ & 0 & 3 & $\mathbb{Z}, 0,0$ & $\mathbb{Z}, 0,0$ & $\mathbb{Z}, \mathbb{Z}, \mathbb{Z}$ & $0,0,0$ \\
\hline $\mathrm{BDI}^{\mathcal{S}_{-+}}$ & 1 & 3 & $\mathbb{Z}_{2}, 0,0$ & $\mathbb{Z}_{2}, 0,0$ & $\mathbb{Z}_{2}, 0,0$ & $2 \mathbb{Z}, 0,0$ \\
\hline $\mathrm{D}^{\mathcal{C S} \mathcal{S}_{-}}$ & 2 & 3 & $\mathbb{Z}_{2}, 0,0$ & $\mathbb{Z}_{2}, 0,0$ & $\mathbb{Z}_{2}, 0,0$ & $0,0,0$ \\
\hline $\mathrm{DIII}^{\mathcal{S}_{+-}}$ & 3 & 3 & $0,0,0$ & $0,0,0$ & $0,0,0$ & $\mathbb{Z}_{2}, \mathbb{Z}_{2}, \mathbb{Z}_{2}$ \\
\hline $\mathrm{AII}^{\mathcal{C} \mathcal{S}_{+}}$ & 4 & 3 & $2 \mathbb{Z}, 0,0$ & $2 \mathbb{Z}, 0,0$ & $2 \mathbb{Z}, 2 \mathbb{Z}, 2 \mathbb{Z}$ & $0,0,0$ \\
\hline $\mathrm{CII}^{\mathcal{S}_{-+}}$ & 5 & 3 & $0,0,0$ & $0,0,0$ & $0,0,0$ & $2 \mathbb{Z}, \mathbb{Z}_{2}, \mathbb{Z}_{2}$ \\
\hline $\mathrm{C}^{\mathcal{C S} \mathcal{S}_{-}}$ & 6 & 3 & $0,0,0$ & $0,0,0$ & $0,0,0$ & $0,0,0$ \\
\hline $\mathrm{CI}^{\mathcal{S}_{+-}}$ & 7 & 3 & $0,0,0$ & $0,0,0$ & $0,0,0$ & $0,0,0$ \\
\hline
\end{tabular}
classification groups are given in the order $\mathcal{D}_{0}^{\prime \prime \prime}, \mathcal{K}_{2}^{\prime \prime \prime}, \mathcal{K}_{1}^{\prime \prime \prime}=\mathcal{K}_{a}^{\prime \prime \prime}$. 
where the symbol " $\hookrightarrow$ " denotes an injection. Since $K^{\prime}\left(d+1, d_{\|}+1\right)=\omega\left[K\left(d, d_{\|}\right)\right]$, it follows that

$$
\operatorname{ker} \omega^{k}= \begin{cases}\operatorname{ker} \omega & \text { for } 0<k \leq q, \\ K\left(d, d_{\|}\right) & \text {for } k>q,\end{cases}
$$

where $\operatorname{ker} \omega \subseteq K\left(d, d_{\|}\right)$. The cut-off $q$ can be obtained from the calculation of ker $\omega$; see Appendix D.

Once $\operatorname{ker} \omega^{k}$ and $K^{\prime}$ are known, the boundary classification groups $\mathcal{K}_{k}^{(n+1)}$ follow from Eq. (30), whereas the subgroup sequence of bulk classification groups follows from the bulk-boundary correspondence (2). The results of this calculation are summarized in Tables II-VII for the bulk classifying sequence for $d=0,1,2$, and 3 , and in Tables X-XII for the boundary classifying groups for $d=3$. Tables with boundary classifying groups for $d=2$ can be found in Ref. [41].

\section{ORDER-RAISING HOMOMORPHISM $\omega$}

In this section, we give an explicit expression for the order-raising homomorphism $\omega$ in terms of the dimensionraising maps $\kappa_{\|}$and $\rho_{\|}$introduced by Shiozaki and Sato [21] and the boundary map $\delta$ of Turner et al., give explicit expressions for the action of $\omega$ on the Hamiltonians $H(\boldsymbol{k}, m)$ introduced in Sec. II, and discuss the relation between $\omega$ and the "layer-stacking construction" $[20,48,53,54]$, which was previously used to construct topological crystalline phases and classify the boundary states. The expression of $\omega$ in terms of the maps $\kappa_{\|}, \rho_{\|}$, and $\delta$ relates it to the $K$-theory approaches to the classification of topological crystalline phases. It plays a key role for establishing the properties of the homomorpishm used in our demonstration of the bulk-boundary correspondence. On the other hand, the explicit realization of the orderraising homomorphism and its relation to the layer-stacking construction are of more use for concrete examples.

\section{A. Construction using dimension-raising isomorphisms}

The order-raising homomorphism $\omega$ is obtained by sequential application of the dimension-raising maps $\kappa_{\|}$ and $\rho_{\|}$of Shiozaki and Sato [21] (see also Sec. II and Appendix B) and the boundary map $\delta$ of Turner et al. [6],

$$
\omega=\kappa_{\|} \circ \delta \circ \rho_{\|} .
$$

Here, the dimension-raising isomorphism $\rho_{\|}$maps an equivalence class of Hamiltonians $H$ to a one-parameter family $H(\varphi), 0 \leq \varphi \leq 2 \pi$, with $H(0)=H(2 \pi)$, on which the crystalline symmetry $\mathcal{S}$ acts nonlocally, $\sigma_{\mathcal{S}} U_{\mathcal{S}} H(\varphi) U_{\mathcal{S}}^{\dagger}=$ $H(2 \pi-\varphi)$; the boundary map $\delta$ then maps the equivalence class of one-parameter families $H(\varphi)$ to

$$
\delta[H(\varphi)]=H(\pi) \ominus H(0),
$$

which gives a Hamiltonian with the topological numbers equal to the difference between the topological numbers of $H(\varphi)$ at $\varphi=0, \pi$. (The operation " $\ominus$ " formally requires the use of the Grothendieck construction; see, e.g., Refs. [6,57].) Last, the dimension-raising isomorphism $\kappa_{\|}$maps the equivalence class of $d$-dimensional Hamiltonians thus obtained to an equivalence class of $(d-1)$-dimensional Hamiltonians, thus defining an element in the group $K\left(d+1, d_{\|}+1\right)$. Although the dimension-raising isomorphisms $\rho_{\|}$and $\kappa_{\|}$also change the Shiozaki-Sato symmetry class, the symmetry class is not changed by combination of the two maps in Eq. (34).

The maps $\kappa_{\|}, \rho_{\|}$, and $\delta$ all respect the group structure of the classifying groups and they commute with the dimensional-raising maps $\kappa_{\|}$and $\kappa_{\perp}$, immediately proving the second property of the order-raising homomorphism advertised in the previous section. A proof of the remaining two properties is given in Appendix C.

\section{B. Explicit realization of the order-raising homomorphism $\omega$}

We recall that the group structure of the bulk and boundary classifying groups is given by the Grothendieck construction. As discussed in Sec. II this motivates us to consider $m$-dependent Hamiltonians $H(\boldsymbol{k}, m)$, such that $H(\boldsymbol{k}, m)$ is in well-defined topological phases for $-2<m<0$ and for $0<m<2$, with the transition between topological classes (if any) taking place at $m=0$. The canonical-form Hamiltonians of Sec. III are examples of such $m$-dependent Hamiltonians. The action of the order-raising homomorphism $\omega$ on Hamiltonians $H(\boldsymbol{k}, m)$ follows from the known action of the maps $\kappa_{\|}$, $\rho_{\|}$, and $\delta$ on such $m$-dependent Hamiltonians $H(\boldsymbol{k}, m)$ $[21,52]$. Specifically, for an equivalence class containing the $d$-dimensional Hamiltonian $H(\boldsymbol{k}, m)$, the mapped class is represented by the Hamiltonian [22]

$$
\omega[H(\boldsymbol{k}, m)]=H_{\omega}\left(\boldsymbol{k}, m+1-\cos k^{\prime}\right)+\Gamma_{\omega} \sin k^{\prime},
$$

where the pair $\left(H_{\omega}, \Gamma_{\omega}\right)$ is given in Tables XIII-XV and the $(d+1)$-dimensional momentum is defined as $\left(k^{\prime}, \boldsymbol{k}\right)$.

TABLE XIII. The action (36) of the order-raising homomorphism $\omega$ on a Hamiltonian $H$ in the complex tenfold-way classes with a unitary order-two symmetry or antisymmetry. $M_{n}$ is the crystalline-symmetry-breaking mass term generated by the homomorphism $\omega$.

\begin{tabular}{lccccc}
\hline \hline TF class & $\mathcal{S}$ & $\left(H_{\omega}, \Gamma_{\omega}\right)$ & $\omega\left(U_{\mathcal{C}}\right)$ & $\omega\left(U_{\mathcal{S}}\right)$ & $M_{n}$ \\
\hline A & $\mathcal{S}$ & $\left(\tau_{3} H, \tau_{1}\right)$ & $\ldots$ & $\tau_{3} U_{\mathcal{S}}$ & $\tau_{2}$ \\
AIII & $\mathcal{S}_{+}$ & $\left(\tau_{3} H, \tau_{2}\right)$ & $\tau_{1}$ & $\tau_{1} U_{\mathcal{C}} U_{\mathcal{S}}$ & $\tau_{3} U_{\mathcal{C}}$ \\
A & $\mathcal{C} \mathcal{S}$ & $\left(\tau_{3} H, \tau_{1}\right)$ & $\ldots$ & $\tau_{0} U_{\mathcal{S}}$ & $\tau_{2}$ \\
AIII & $\mathcal{S}_{-}$ & $\left(\tau_{3} H, \tau_{2}\right)$ & $\tau_{1}$ & $\tau_{3} U_{\mathcal{S}}$ & $\tau_{3} U_{\mathcal{C}}$ \\
\hline \hline
\end{tabular}


TABLE XIV. The action (36) of the order-raising homomorphism $\omega$ on a Hamiltonian in the complex tenfold-way classes with an antiunitary order-two symmetry or antisymmetry. $M_{n}$ is the crystalline-symmetry-breaking mass term generated by the homomorphism $\omega$.

\begin{tabular}{lccccc}
\hline \hline TF class & $\mathcal{S}$ & $\left(H_{\omega}, \Gamma_{\omega}\right)$ & $\omega\left(U_{\mathcal{C}}\right)$ & $\omega\left(U_{\mathcal{S}}\right)$ & $M_{n}$ \\
\hline A & $\mathcal{T}^{+} \mathcal{S}, \mathcal{T}^{-} \mathcal{S}$ & $\left(\tau_{3} H, \tau_{1}\right)$ & $\ldots$ & $\tau_{0} U_{\mathcal{S}}$ & $\tau_{2}$ \\
AIII & $\mathcal{P}^{+} \mathcal{S}_{+}, \mathcal{P}^{-} \mathcal{S}_{+}$ & $\left(\tau_{3} H, \tau_{2}\right)$ & $\tau_{1}$ & $\tau_{0} U_{\mathcal{S}}$ & $\tau_{3} U_{\mathcal{C}}$ \\
A & $\mathcal{P}^{+} \mathcal{S}, \mathcal{P}^{-} \mathcal{S}$ & $\left(\tau_{3} H, \tau_{1}\right)$ & $\ldots$ & $\tau_{3} U_{\mathcal{S}}$ & $\tau_{2}$ \\
AIII & $\mathcal{T}^{+} \mathcal{S}_{-}, \mathcal{T}^{-} \mathcal{S}_{-}$ & $\left(\tau_{3} H, \tau_{2}\right)$ & $\tau_{1}$ & $\tau_{3} U_{\mathcal{S}}$ & $\tau_{3} U_{\mathcal{C}}$ \\
\hline \hline
\end{tabular}

The maps $\kappa_{\|}, \delta$, and $\rho_{\|}$featuring in the definition (34) can be represented in a similar way; see Appendix C.

\section{Stacking construction}

References [53-55] construct higher-order topological phases by stacking layers of lower-dimensional ones. Like the order-raising homomorphism $\omega$ considered here, the stacking construction also involves simultaneously increasing the spatial dimension $d$ and the number of inverted dimensions $d_{\|}$by one, so that it, too, provides a homomorphism $\sigma$

$$
\sigma: K\left(d, d_{\|}\right) \rightarrow K\left(d+1, d_{\|}+1\right) .
$$

Further, in Ref. [55] it is argued, from the boundary perspective, that the stacking of $d$-dimensional "layers" that differ by a separable phase yields topologically equivalent $(d+1)$-dimensional crystals. This, too, is a property that is shared by the order-raising homomorphism $\omega$. Indeed, below we show that the stacking homomorphism $\sigma$ has all three defining properties of the order-raising homomorphism specified in Sec. IV. The order-raising homomorphism $\omega$ of Sec. VA and the stacking construction are two realizations of the same homomorphism.

Specifically, the stacking procedure constructs a $(d+1)$ dimensional crystal by alternating $d$-dimensional layers

TABLE XV. The action (36) of the order-raising homomorphism $\omega$ on a Hamiltonian in the real tenfold-way classes with a unitary order-two symmetry or antisymmetry. $M_{n}$ is the crystalline-symmetry-breaking mass term generated by the homomorphism $\omega$.

\begin{tabular}{lcccccc}
\hline \hline TF classes & $\mathcal{S}$ & $\left(H_{\omega}, \Gamma_{\omega}\right)$ & $\omega\left(U_{\mathcal{T}}\right)$ & $\omega\left(U_{\mathcal{P}}\right)$ & $\omega\left(U_{\mathcal{S}}\right)$ & $M_{n}$ \\
\hline AI, AII & $\mathcal{S}_{+}, \mathcal{S}_{-}$ & $\left(\tau_{3} H, \tau_{1}\right)$ & $\tau_{3} U_{\mathcal{T}}$ & $\ldots$ & $\tau_{3} U_{\mathcal{S}}$ & $\tau_{2}$ \\
AI, AII & $\mathcal{C S}_{+}, \mathcal{C} \mathcal{S}_{-}$ & $\left(\tau_{3} H, \tau_{1}\right)$ & $\tau_{3} U_{\mathcal{T}}$ & $\ldots$ & $\tau_{0} U_{\mathcal{S}}$ & $\tau_{2}$ \\
BDI, CII & $\mathcal{S}_{++}, \mathcal{S}_{--}\left(\tau_{3} H, \tau_{2}\right)$ & $\tau_{0} U_{\mathcal{T}}$ & $\tau_{1} U_{\mathcal{T}}$ & $\tau_{1} U_{\mathcal{C}} U_{\mathcal{S}}$ & $\tau_{3} U_{\mathcal{C}}$ \\
BDI, CII & $\mathcal{S}_{+-}, \mathcal{S}_{-+}\left(\tau_{3} H, \tau_{2}\right)$ & $\tau_{0} U_{\mathcal{T}}$ & $\tau_{1} U_{\mathcal{T}}$ & $\tau_{3} U_{\mathcal{S}}$ & $\tau_{3} U_{\mathcal{C}}$ \\
D, C & $\mathcal{S}_{+}, \mathcal{S}_{-}$ & $\left(\tau_{3} H, \tau_{1}\right)$ & $\ldots$ & $\tau_{0} U_{\mathcal{P}}$ & $\tau_{3} U_{\mathcal{S}}$ & $\tau_{2}$ \\
D, C & $\mathcal{C S}_{+}, \mathcal{C} \mathcal{S}_{-}\left(\tau_{3} H, \tau_{1}\right)$ & $\ldots$ & $\tau_{0} U_{\mathcal{P}}$ & $\tau_{0} U_{\mathcal{S}}$ & $\tau_{2}$ \\
DIII, CI & $\mathcal{S}_{++}, \mathcal{S}_{--}\left(\tau_{3} H, \tau_{2}\right)$ & $\tau_{2} U_{\mathcal{P}}$ & $\tau_{3} U_{\mathcal{P}}$ & $\tau_{1} U_{\mathcal{C}} U_{\mathcal{S}}$ & $\tau_{3} U_{\mathcal{C}}$ \\
DIII, CI & $\mathcal{S}_{+-}, \mathcal{S}_{-+}\left(\tau_{3} H, \tau_{2}\right)$ & $\tau_{2} U_{\mathcal{P}}$ & $\tau_{3} U_{\mathcal{P}}$ & $\tau_{3} U_{\mathcal{S}}$ & $\tau_{3} U_{\mathcal{C}}$ \\
\hline \hline
\end{tabular}

(a)

(b)

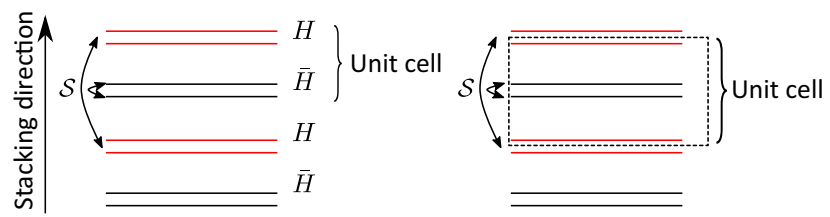

FIG. 6. Layer-stacking construction of higher-order topological phases: (a) A $(d+1)$-dimensional crystal is constructed out of alternating $d$-dimensional layers with opposite topological numbers. The unit cell consisting of two such layers is not mapped to itself under the (anti)symmetry operation $\mathcal{S}$, which inverts the coordinate $x_{d+1}$ in the stacking direction. (b) The unit cell may be redefined, so that it is mapped to itself under $\mathcal{S}$. This redefinition of the unit cell involves splitting the odd layers into two parts that are mapped onto each other under $\mathcal{S}$, eventually after adding topological trivial bands.

with opposite topological numbers as shown schematically in Fig. 6(a). Denoting the Hamiltonians of the alternating $d$-dimensional layers as $H_{d}(\boldsymbol{k})$ and $\bar{H}_{d}(\boldsymbol{k})$, respectively, the Hamiltonian of the $(d+1)$-dimensional stack is

$$
H_{d+1}\left(\boldsymbol{k}, k_{d+1}\right)=\left(\begin{array}{cc}
H_{d}(\boldsymbol{k}) & 0 \\
0 & \bar{H}_{d}(\boldsymbol{k})
\end{array}\right) .
$$

If the $d$-dimensional Hamiltonians $H_{d}$ and $\bar{H}_{d}$ have a crystalline (anti)symmetry with $d_{\|}$inverted dimensions encoded by the unitary matrix $U_{\mathcal{S}}$, the $(d+1)$-dimensional Hamiltonian $H_{d+1}$ has two crystalline (anti)symmetries, encoded by $\operatorname{diag}\left(U_{\mathcal{S}}, U_{\mathcal{S}}\right)$ and $\operatorname{diag}\left(e^{i k_{d+1}} U_{\mathcal{S}}, U_{\mathcal{S}}\right)$, with $d_{\|}$ and $d_{\|}+1$ inverted dimensions, respectively. The former (anti)symmetry yields a weak topological crystalline phase and will not be considered here. The latter (anti)symmetry has a $k_{d+1}$-dependent transformation matrix, which reflects the fact that it does not map the unit cell defined by the representation (37) of $H_{d+1}$ to itself, see Fig. 6(a). To remedy this situation we replace Eq. (37) by

$$
\begin{aligned}
\sigma\left(H_{d}\right) & \equiv\left(\begin{array}{cc}
H_{d+1}^{\prime}\left(\boldsymbol{k}, k_{d+1}\right) & 0 \\
0 & \bar{H}_{d}(\boldsymbol{k})
\end{array}\right) \\
& =\left(\begin{array}{cc}
e^{i \hat{\rho} k_{d+1} / 2} H_{d}(\boldsymbol{k}) e^{-i \hat{\rho} k_{d+1} / 2} & 0 \\
0 & \bar{H}_{d}(\boldsymbol{k})
\end{array}\right),
\end{aligned}
$$

where $\hat{\rho}$ is a matrix that commutes with the nonspatial (anti)symmetries $\mathcal{T}, \mathcal{P}$, and $\mathcal{C}$, and anticommutes with $U_{\mathcal{S}}$, and the crystalline (anti)symmetry is represented by $\operatorname{diag}\left(U_{\mathcal{S}}, U_{\mathcal{S}}\right)$. (Being able to find a matrix $\hat{\rho}$ with these properties may require the addition of additional, topological trivial bands.) Loosely speaking, the transformation described by Eq. (38) involves the redefinition of the unit cell as in Fig. 6(b), so that the additional crystalline symmetry $\mathcal{S}$ maps the $(d+1)$-dimensional unit cell to itself for the new choice of the unit cell. 
The form of the bulk Hamiltonian (38) immediately allows us to conclude that for $H_{d}(\boldsymbol{k})$ separable, the Hamiltonian $H_{d}(\boldsymbol{k})$ can be deformed to manifestly separable form and the matrix $\hat{\rho}$ can be chosen to commute with it, resulting in a $k_{d+1}$-independent, and therefore topologically trivial Hamiltonian $\sigma\left(H_{d}\right)$ (aside from possible weak invariants). The reverse is also true: $\sigma\left(H_{d}\right)$ topologically trivial implies that the upper-right block $H_{d+1}^{\prime}$ of Eq. (38) has only weak topological invariants. Thus, $H_{d+1}^{\prime}$ can be continuously deformed to a $k_{d+1}$-independent Hamiltonian. The only possible way to remove $k_{d+1}$ dependence from $e^{i \hat{\rho} k_{d+1} / 2} H_{d}(\boldsymbol{k}) e^{-i \hat{\rho} k_{d+1} / 2}$ is to continuously deform the Hamiltonian $H_{d}$ and/or the matrix $\rho$ to mutually commute. We have therefore shown that $\sigma(H)$ is in the trivial class if and only if $H$ is separable.

The above statement is obtained from the bulk perspective; accordingly, it also holds for $d$-dimensional topological phases from $K^{(d)}$ that do not support topologically protected boundary states.

The $d$-dimensional Hamiltonian $H_{d}(\boldsymbol{k})$ in Eq. (38) is to be understood as an $m$-dependent family $H_{d}(\boldsymbol{k}, m)$ that represents a topologically trivial Hamiltonian for $m>0$. A topologically trivial Hamiltonian is separable, and we choose a parametrization where $H_{d}(\boldsymbol{k}, m)$ is manifestly separable for $m>0$. With this choice, the term $e^{i \hat{\rho} k_{d+1} / 2} H_{d}(\boldsymbol{k}, m) e^{-i \hat{\rho} k_{d+1} / 2}$ is $k_{d+1}$ independent for $m>0$, thus trivial without any additional weak invariants.

Using the definition (38) and Eq. (36) applied to the dimension-raising maps $\kappa_{\|}$and $\kappa_{\perp}$, i.e., with $\omega$ replaced by $\kappa_{\|}$or $\kappa_{\perp}$, we obtain the following: the stacking homomorphism $\sigma$ commutes with the dimension-raising isomorphisms $\kappa_{\|}$and $\kappa_{\perp}$.

The stacking construction has the property that if a nonseparable Hamiltonian $H_{d}$ supports topologically protected states on its $(d-1)$-dimensional boundary, $\sigma(H)$ also supports topologically protected states of the same dimensionality on its $d$-dimensional boundary, see Refs. [53-55] - combined with the above property this gives the following: If $H$ is a nonseparable Hamiltonian with $n-1$ crystalline-symmetry-breaking mass terms, then $\sigma(H)$ is a Hamiltonian with $n$ crystalline-symmetrybreaking mass terms.

To see this, consider a $d$-dimensional Hamiltonian $H_{d}$ with $n$ crystalline-symmetry-breaking mass terms. By repeatedly applying the dimension-raising isomorphism $\kappa_{\perp}$ and $\kappa_{\|}$or their inverse, we can change both the values of $d$ and $d_{\|}$to $n+1$. The resulting inversion-symmetric $(n+1)$-dimensional Hamiltonian $H_{n+1}$ is guaranteed to have zero-dimensional protected boundary states; see Sec. III. Thus, $\sigma\left(H_{n+1}\right)$ has also zero-dimensional topologically protected boundary states [53-55], and accordingly $\sigma\left(H_{n+1}\right)$ has $n+1$ crystalline-symmetry-breaking mass terms (boundary mass terms). Since the homomorphism $\sigma$ commutes with the dimensionraising isomorphism $\kappa_{\perp}$ and $\kappa_{\|}$, the same is true for $\sigma\left(H_{d}\right)$.
We additionally checked that $\omega\left(H_{0}\right) \cong \sigma\left(H_{0}\right)$ for zerodimensional Hamiltonians $H_{0}$.

Although the realizations $\sigma$ and $\omega$ are indistinguishable as homomorphisms between classifying groups, their action on a specific Hamiltonian is rather different. When acting on a nearest-neighbor hopping Hamiltonian, the homomorphism $\omega$ gives a Hamiltonian of the same form. In particular, if $H$ is a minimal canonical-form Hamiltonian, $\omega(H)$ is also a minimal canonical-form Hamiltonian. On the other hand, as is evident from the definition (38), the stacking homomorphism $\sigma$ generates hopping elements beyond the nearest neighbors. Section VI illustrates these differences for three examples.

\section{EXAMPLES}

In this section, we illustrate the full classification using the subgroup sequence (1) for a few representative examples and show how the order-raising homomorphism $\omega$ relates topological crystalline phases in different dimensions. We further compare the realization of the orderraising homomorphism $\omega$, see Sec. VA and Appendix C, to that of the layer-stacking procedure of Refs. [20,48,53,54]; see Sec. V C. Additionally, we discuss the connection to recently studied embedded topological phases [65]. As in the previous section, we reserve the symbol $\omega$ for the concrete realization of the order-raising homomorphism given in Secs. VA and V B.

The models we consider can all be expressed in the canonical form of Eq. (10), where we add the perturbation (12) or a crystalline-symmetry-breaking mass term localized at the sample boundaries to gap out the boundaries (if applicable). The action of the order-raising homomorphism $\omega$ on $H_{0}$ is defined by Eq. (36) and Tables XIII-XV.

\section{A. Higher-order phases originating from the quantum Hall phase}

In two dimensions, systems with broken time-reversal symmetry but without crystalline symmetries admit a quantum Hall phase, which has chiral propagating modes along crystal edges. This first-order topological phase in tenfold-way class A is compatible with an on-site crystalline symmetry $\mathcal{O}$, with a mirror antisymmetry $\mathcal{C M}$, and with a twofold rotation symmetry $\mathcal{R}$. Further, in the presence of $\mathcal{C M}$ a two-dimensional second-order topological phase with protected zero-energy states at mirrorsymmetric corners is possible, too. The order-raising homomorphism links these two-dimensional topological phases to three-dimensional topological phases with an additional mirror symmetry $\mathcal{M}$, rotation antisymmetry $\mathcal{C} \mathcal{R}$, or inversion symmetry $\mathcal{I}$, respectively. For each of these cases we describe the action of the order-raising homomorphism in detail and show how it connects the subgroup sequences classifying the bulk crystalline topology for the two-dimensional and three-dimensional phases. 
Higher-order phases originating from class $A^{\mathcal{O}}$ in two dimensions. - Without loss of generality we may represent the on-site crystalline symmetry $\mathcal{O}$ using $U_{\mathcal{O}}=\tau_{3}$. The onsite crystalline symmetry forces the Hamiltonian $H$ to have a block-diagonal structure, $H=\operatorname{diag}\left(h_{+}, h_{-}\right)$, with separate blocks $h_{+}$and $h_{-}$for even and odd-parity states, respectively. In two dimensions the bulk band structure in class $\mathrm{A}^{\mathcal{O}}$ is classified by the subgroup sequence $0 \subseteq 0 \subseteq \mathbb{Z}^{2}$; see Table II. The existence of first-order topological phases with a $\mathbb{Z}^{2}$ classification follows directly from the well-known $\mathbb{Z}$ classification for the tenfold-way class A, because the individual blocks $h_{ \pm}$are not subject to any constraints from the crystalline symmetry. The minimal canonical-form generators for the $\mathbb{Z}^{2}$ classifying group are quantum-Hall phases for each parity block separately, which have $\Gamma_{0}=\sigma_{1}, \boldsymbol{\Gamma}=\left(\sigma_{2}, \sigma_{3}\right)$, i.e., Hamiltonians

$$
\begin{aligned}
h_{ \pm}(\boldsymbol{k}, m)= & \sigma_{2}\left(2+m-\cos k_{x}-\cos k_{y}\right) \\
& +\sigma_{3} \sin k_{x}+\sigma_{1} \sin k_{y} .
\end{aligned}
$$

(The Pauli matrices $\sigma_{j}$ act on a different d.o.f. than the Pauli matrix $\tau_{3}$ used to represent the on-site crystalline symmetry.) Applying the order-raising homomorphism $\omega$ to such a generator gives a three-dimensional canonical-form Hamiltonian with (see Table XIII)

$$
\Gamma_{0}=\tau_{3} \sigma_{2}, \quad \boldsymbol{\Gamma}=\left(\tau_{1}, \tau_{3} \sigma_{3}, \tau_{3} \sigma_{1}\right),
$$

which satisfies an additional mirror symmetry with the representation $U_{\mathcal{M}}=\tau_{3}$. This Hamiltonian has one crystalline-symmetry-breaking mass term $M_{1}=\tau_{2}$, so that it represents a second-order topological phase.

One verifies that two-dimensional Hamiltonians for class $\mathrm{A}^{\mathcal{O}}$ are separable if and only if the even and odd parity blocks have equal $\mathbb{Z}$ topological indices. This implies ker $\omega=\mathbb{Z}$, so that the image of the classifying group $\omega[K(2,0)]=K^{\prime}(3,1)=\mathbb{Z}$. [We recall our notation according to which the groups $K^{(n)}\left(d, d_{\|}\right)$classify the bulk topology for $d$-dimensional phases with a crystalline symmetry with $d_{\|}$inverted dimensions.] Since the tenfold-way class $\mathrm{A}$ is trivial for $d=3$ there are no first-order phases, i.e., $K(3,1)=K^{\prime}(3,1)$, consistent the subgroup sequence $0 \subseteq 0 \subseteq \mathbb{Z} \subseteq \mathbb{Z}$ for class $\mathrm{A}^{\mathcal{M}}$ in three dimensions; see Table V.

From the boundary perspective, for class $\mathrm{A}^{\mathcal{M}}$ in three dimensions one finds $\mathcal{D}_{0}^{\prime \prime}(3,1)=\mathbb{Z}^{2}$, where the two $\mathbb{Z}$ indices counts the number of chiral hinge modes for each mirror parity. Equal numbers of chiral hinge modes for the two mirror parities correspond to a separable boundary phase, so that $\mathcal{D}_{1}^{\prime \prime}(3,1)=\mathbb{Z}$. The anomalous boundary classifying group is, hence, $\mathcal{K}_{a}^{\prime \prime}(3,1)=\mathcal{D}_{0}^{\prime \prime}(3,1) / \mathcal{D}_{1}^{\prime \prime}(3,1)=\mathbb{Z}[28]$.

Higher-order phases originating from class $A^{\mathcal{C M}}$ in two dimensions.-This class has a $0 \subseteq \mathbb{Z} \subseteq \mathbb{Z}^{2}$ subgroup sequence for its bulk topological classification in two dimensions; see Table II. For definiteness we choose to represent the mirror antisymmetry by $U_{\mathcal{C M}}=\sigma_{3}$, so that

$$
H\left(k_{x}, k_{y}\right)=-\sigma_{3} H\left(-k_{x}, k_{y}\right) \sigma_{3} .
$$

At the high-symmetry lines $k_{x}=0$ or $\pi$ the mirror antisymmetry $\mathcal{C M}$ effectively simplifies to a chiral antisymmetry $\mathcal{C}$ represented by $U_{\mathcal{C}}=\sigma_{3}$, allowing one to define the difference $W$ of winding numbers for $0 \leq k_{y} \leq 2 \pi$ at $k_{x}=0$ and $k_{x}=\pi$ as a suitable topological index. The second topological invariant of the Hamiltonian $H$ is the Chern number $C$, which counts the number of chiral boundary modes. Since $C$ and $W$ have the same parity, the $\mathbb{Z}^{2}$ bulk topological index $(p, q)$ can be defined setting $p=(C+W) / 2, q=(C-W) / 2$. A common set of generators for the classifying groups $K(2,1)=\mathbb{Z}^{2}$ and $K^{\prime}(2,1)=\mathbb{Z}$ is given by the canonical-form Hamiltonians $H_{(1,0)}$ and $H_{(1,-1)}$, with

$$
\begin{array}{lll}
\Gamma_{0}=\sigma_{2}, & \boldsymbol{\Gamma}=\left(\sigma_{3}, \sigma_{1}\right), & \text { for } H_{(1,0)}, \\
\Gamma_{0}=\sigma_{2} \tau_{0}, & \boldsymbol{\Gamma}=\left(\sigma_{3} \tau_{3}, \sigma_{1} \tau_{0}\right), & \text { for } H_{(1,-1)} .
\end{array}
$$

The Hamiltonian $H_{(1,0)}$ represents a first-order topological phase with a single anomalous chiral boundary mode; it is a generator of $K(2,1)$, but not of $K^{\prime}(2,1)$. The Hamiltonian $H_{(1,-1)}$, which has the $\mathcal{C} \mathcal{M}$-breaking mass terms $M_{1}=$ $\sigma_{3} \tau_{1}$ and $M_{2}=\sigma_{3} \tau_{2}$, represents a second-order topological phase, with anomalous zero-energy corner states at mirrorsymmetric corners; it is a generator of both $K(2,1)$ and $K^{\prime}(2,1)$; see Fig. 7.

The order-raising homomorphism $\omega$ maps a twodimensional Hamiltonian with a mirror antisymmetry $\mathcal{C M}$ to a three-dimensional Hamiltonian with a rotation antisymmetry $\mathcal{C} \mathcal{R}$. To see how the order-raising map $\omega$ maps between the subgroup sequence of the two classes, we first consider its action on the Hamiltonian $H_{(1,0)}$ considered above. Application of the order-raising homomorphism $\omega$ to $H_{(1,0)}$ gives the three-dimensional canonical-form Hamiltonian with

$$
\Gamma_{0}=\sigma_{2} \tau_{3}, \quad \boldsymbol{\Gamma}=\left(\sigma_{0} \tau_{1}, \sigma_{3} \tau_{3}, \sigma_{1} \tau_{3}\right)
$$

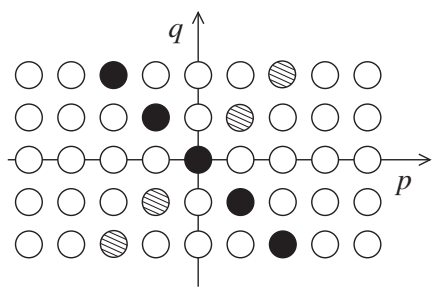

FIG. 7. The full bulk classifying group $K(2,1)$ for classes $\mathrm{A}^{\mathcal{C M}}$ is $\mathbb{Z}^{2}$. The purely crystalline subgroup $K^{\prime}(2,1)$ and the separable subgroup $\operatorname{ker} \omega$ are denoted by solid and hatched circles, respectively. 
which satisfies a twofold-rotation antisymmetry $\mathcal{C R}$ with the representation $U_{\mathcal{C R}}=\sigma_{2}$. This Hamiltonian has a single crystalline-symmetry-breaking mass term $M_{1}=\tau_{2}$, corresponding to a second-order topological phase with a single chiral mode along a hinge. To further specify the action of the order-raising homomorphism $\omega$, we search for separable two-dimensional Hamiltonians, since these are mapped to the trivial class under $\omega$. With a little algebra one verifies that the canonical-form Hamiltonian with

$$
\Gamma_{0}=\sigma_{2} \tau_{0}, \quad \boldsymbol{\Gamma}=\left(\sigma_{3} \tau_{1}, \sigma_{1} \tau_{1}\right)
$$

has topological indices $(p, q)=(1,1)$ and is separable. We thus identify $H_{(1,1)}$ as the generator of the separable subgroup ker $\omega$; see Fig. 7. Since $H_{(2,0)}$ differs from $H_{(1,-1)}$ by a separable Hamiltonian, $H_{(2,0)}$ and $H_{(1,-1)}$ must be mapped to the same topological class under $\omega$, i.e., $\omega\left(H_{(2,0)}\right)$ must be a representative of a third-order topological phase. It follows that $K^{\prime}(3,2)=\omega[K(2,1)]=\mathbb{Z}$ and $K^{\prime \prime}(3,2)=\omega\left[K^{\prime}(2,1)\right]=2 \mathbb{Z}$. Combined with the observation that there are no first-order topological phases for the tenfold-way class $\mathrm{A}$, we arrive at the subgroup sequence $0 \subseteq 2 \mathbb{Z} \subseteq \mathbb{Z} \subseteq \mathbb{Z}$ for three-dimensional Hamiltonians with a twofold-rotation antisymmetry, consistent with Table V.

From the boundary perspective, we note that chiral hinge modes have a $\mathbb{Z}$ classification: The $\mathbb{Z}$ topological index simply counts the number of chiral hinge modes. Hence, $\mathcal{D}_{0}^{\prime \prime}(3,2)=\mathbb{Z}$. The presence of the $\mathcal{C} \mathcal{R}$ antisymmetry plays no role here, as it does not leave any hinges invariant. An even number of hinge modes represents a separable phase, so that $\mathcal{D}_{1}^{\prime \prime}(3,2)=2 \mathbb{Z}$. It follows that $\mathcal{K}_{a}^{\prime \prime}(3,2)=\mathcal{D}_{0}^{\prime \prime}(3,2) /$ $\mathcal{D}_{1}^{\prime \prime}(3,2)=\mathbb{Z}_{2}$. To describe the third-order phases from the boundary perspective, we note that the rotation antisymmetry is a local symmetry for a corner on the rotation axis. For this situation one finds a $\mathbb{Z}$ topological index, counting the difference of the number of zero-energy corner states for even and odd $\mathcal{C} \mathcal{R}$ parity. Since none of these boundary classes is separable, one has $\mathcal{D}_{0}^{\prime \prime \prime}(3,2)=\mathbb{Z}, \mathcal{D}_{1}^{\prime \prime \prime}(3,2)=$ $\mathcal{D}_{2}^{\prime \prime \prime}(3,2)=0$, so that and $\mathcal{K}_{2}^{\prime \prime \prime}(3,2)=\mathcal{K}_{a}^{\prime \prime \prime}(3,2)=\mathbb{Z}$; see Table X.

Higher-order phases originating from class $\mathrm{A}^{\mathcal{R}}$ in two dimensions.-The bulk topological classification of a twodimensional topological insulator with an additional twofold rotation symmetry $\mathcal{R}$ is given by the subgroup sequence $\mathbb{Z} \subseteq \mathbb{Z} \subseteq \mathbb{Z}^{2}$, implying a $\mathbb{Z}$ topological index for first-order phases and a $\mathbb{Z}$ topological index classifying topological phases without boundary states. (Such phases are essentially atomic-limit insulators.) The two topological invariants are the Chern number $C$ and the number $N=n_{o}(0,0)-n_{o}(\pi, 0)-n_{o}(0, \pi)+n_{o}(\pi, \pi)$, where $n\left(k_{x}, k_{y}\right)$ is the number of occupied odd-parity bands at the high-symmetry momentum $\left(k_{x}, k_{y}\right)[16,18,21]$. Since $C$ and $N$ have the same parity, the $\mathbb{Z}^{2}$ bulk topological index $(p, q)$ is defined setting $p=(C-N) / 2, q=(C+N) / 2$. A common set of generators for the classifying groups $K^{(n)}(2,2), n=0,1,2$, is given by the canonical-form Hamiltonians $H_{(1,0)}$ and $H_{(1,-1)}$ of Eq. (42), where we have chosen the representation $U_{\mathcal{R}}=\sigma_{2}$. The Hamiltonian $H_{(1,0)}$, which has the $\mathcal{R}$-breaking mass terms $\sigma_{3} \tau_{1}$ and $\sigma_{3} \tau_{2}$, represents a first-order topological phase with a single anomalous chiral boundary mode; it is a generator of $K(2,2)$, but not of $K^{\prime}(2,2)$ and $K^{\prime \prime}(2,2)$. The Hamiltonian $H_{(1,-1)}$ represents an atomic insulator with no boundary states; it is a generator of $K(2,2), K^{\prime}(2,2)$, and $K^{\prime \prime}(2,2)$.

The order-raising homomorphism $\omega$ maps a twodimensional Hamiltonian with a twofold rotation symmetry $\mathcal{R}$ to a three-dimensional Hamiltonian with inversion symmetry $\mathcal{I}$. To see how the order-raising map $\omega$ maps between the subgroup sequence of the two classes, we first consider its action on the Hamiltonian $H_{(1,0)}$ considered above. Application of the order-raising homomorphism $\omega$ to $H_{(1,0)}$ gives the three-dimensional canonical-form Hamiltonian specified by Eq. (43), which satisfies an inversion symmetry represented by $U_{\mathcal{I}}=\tau_{3} \sigma_{2}$. As in the previous example, one verifies that the canonical-form Hamiltonian with

$$
\Gamma_{0}=\sigma_{2} \tau_{1}, \quad \boldsymbol{\Gamma}=\left(\sigma_{3} \tau_{1}, \sigma_{1} \tau_{0}\right)
$$

has topological indices $(p, q)=(1,1)$ and is separable. Accordingly, $H_{(1,1)}$ is the generator of the subgroup ker $\omega \subseteq K(2,2)$. Since $H_{(1,-1)}$ differs from $H_{(2,0)}$ by a separable Hamiltonian, we conclude that $H_{(2,0)}$ and $H_{(1,1)}$ must be mapped to the same topological class under $\omega$. Since $H_{(1,1)}$ represents an atomic insulator without boundary states, its image $\omega\left(H_{(2,0)}\right)$ must also represent an atomic insulator without boundary states. It follows that $K^{\prime}(3,3)=$ $\omega[K(2,2)]=\mathbb{Z} \quad$ and $\quad K^{\prime \prime}(3,3)=\omega\left[K^{\prime \prime}(2,2)\right]=$ $K^{\prime \prime \prime}(3,3)=\omega\left[K^{\prime \prime \prime}(3,3)\right]=2 \mathbb{Z}$. As in the previous example, since there are no first-order topological phases for the tenfold-way class $\mathrm{A}$, we thus arrive at the subgroup sequence $2 \mathbb{Z} \subseteq 2 \mathbb{Z} \subseteq \mathbb{Z} \subseteq \mathbb{Z}$ for class $A^{\mathcal{I}}$ in three dimensions, consistent with Table V.

We conclude this example with a discussion of the classification from the boundary perspective. We first note that chiral hinge modes have a $\mathbb{Z}$ classification, whereby the $\mathbb{Z}$ topological index simply counts the number of chiral hinge modes. Hence, $\mathcal{D}_{0}^{\prime \prime}(3,2)=\mathbb{Z}$. The presence of the inversion symmetry plays no role here, as $\mathcal{I}$ does not leave any hinges invariant. An even number of hinge modes represents a separable phase, so that $\mathcal{D}_{1}^{\prime \prime}(3,2)=$ $2 \mathbb{Z}$. It follows that $\mathcal{K}_{a}^{\prime \prime}(3,2)=\mathcal{D}_{0}^{\prime \prime}(3,2) / \mathcal{D}_{1}^{\prime \prime}(3,2)=\mathbb{Z}_{2}$. Finally, since no protected zero-energy corner states are possible in the absence of an antisymmetry, the boundary classification of third-order phases is entirely trivial, 
$\mathcal{D}_{0}^{\prime \prime \prime}(3,2)=\mathcal{D}_{1}^{\prime \prime \prime}(3,2)=\mathcal{D}_{2}^{\prime \prime \prime}(3,2)=\mathcal{K}_{2}^{\prime \prime \prime}(3,2)=\mathcal{K}_{a}^{\prime \prime \prime}(3,2)=0 ;$ see Table $X$.

\section{B. Separable higher-order topological phases}

As discussed in Sec. IV, the boundary classification considers classifying groups $\mathcal{K}_{k}^{(n)}$ for protected states at boundaries of dimension $d-n$, whereby such boundary states are considered equivalent if they differ by a lattice termination along a boundary of dimension $\leq d-k$. The boundary classifying groups $\mathcal{K}_{k}^{(n)}=\mathcal{D}_{0}^{(n)} / \mathcal{D}_{k}^{(n)}$ are the quotient of the group $\mathcal{D}_{0}^{(n)}$ classifying all possible $(d-n)$-dimensional boundary states and the "decoration subgroup" $\mathcal{D}_{k}^{(n)}$, which classifies $(d-n)$-dimensional boundary states that can be attributed to the combination of a topological nontrivial boundary of dimension $\leq d-k$ and a topologically trivial bulk.

In the examples of the previous subsection, all decoration groups $\mathcal{D}_{k}^{(n)}$ with $k=2, \ldots, n$ are equal, so that effectively it is sufficient to consider the (a priori) smallest decoration subgroup $\mathcal{D}_{n-1}^{(n)}$, which classifies the $(d-n)$ dimensional boundary states of a symmetry-compatible $(d-n+1)$-dimensional topological phase located on the crystal boundary. This $(d-n+1)$-dimensional topological phase is a separable phase, i.e., it consists of two halves, which are mapped onto each other by the crystalline symmetry $\mathcal{S}$; see Fig. 5. Although the group $\mathcal{D}_{n-1}^{(n)}$ describes codimension- $n$ boundary states of the crystal as a whole, it describes first-order boundary states of the separable $(d-n+1)$-dimensional topological phase located on the crystal boundary.

There are seven Shiozaki-Sato symmetry classes, for which the decoration subgroups $\mathcal{D}_{k}^{(n)}$ are not the same for all $k$. For those classes, the integer $q$ in Eq. (33) is finite and one must consider higher-order separable phases to obtain the boundary classification. Five of these classes are relevant for the boundary classification of third-order phases in three dimensions. These classes originate from two-dimensional separable second-order phases in classes DIII ${ }^{\mathcal{M}_{++}}$, DIII ${ }^{\mathcal{M}_{-+}}$, $\mathrm{D}^{\mathcal{M}_{+}}, \mathrm{AII}^{\mathcal{C} \mathcal{M}_{-}}$, and $\mathrm{A}^{\mathcal{P}^{+} \mathcal{M}}$. Two of these classes are relevant for the boundary classification of fourth-order phases in four dimensions. These classes can be traced to separable threedimensional third-order phases in classes $\mathrm{CII}^{\mathcal{R}_{--}}$and AIII ${ }^{\mathcal{T}^{+} \mathcal{R}_{+}}$. We now discuss two of these classes in detail.

Class $\mathrm{DIII}^{\mathcal{M}_{++}}$in two dimensions.-We choose the representation $U_{\mathcal{T}}=\sigma_{2}$ and $U_{\mathcal{P}}=\tau_{1}$ for time-reversal and particle-hole conjugation, respectively. Starting from the tenfold-way canonical-form Hamiltonian specified by

$$
\Gamma_{0}=\sigma_{0} \tau_{3}, \quad \boldsymbol{\Gamma}=\left(\sigma_{1} \tau_{1}, \sigma_{0} \tau_{2}\right),
$$

which has a single helical Majorana boundary mode, we construct the manifestly separable Hamiltonian
$\operatorname{diag}\left[H\left(k_{1}, k_{2}\right), \sigma_{1} H\left(-k_{1}, k_{2}\right) \sigma_{1}\right]$, which has the canonical-form representation

$$
\Gamma_{0}=\mu_{0} \sigma_{0} \tau_{3}, \quad \boldsymbol{\Gamma}=\left(\mu_{3} \sigma_{1} \tau_{1}, \mu_{0} \sigma_{0} \tau_{2}\right),
$$

where the $\mu_{j}$ are Pauli matrices acting on a different d.o.f. than the Pauli matrices $\sigma_{j}$ and $\tau_{j}$. The Hamiltonian (47) satisfies the mirror symmetry $U_{\mathcal{M}}=\sigma_{1} \mu_{1}$, which commutes with $\mathcal{T}$ and $\mathcal{P}$. It has a single $\mathcal{M}$-breaking mass term $M=\mu_{2} \sigma_{1} \tau_{1}$, which makes it a second-order topological superconductor with protected zero-energy states at the two mirror-symmetric corners.

Two-dimensional separable Hamiltonians can be used to decorate a three-dimensional bulk, as shown schematically in Fig. 8. Specifically, the separable two-dimensional system is deformed into a two-dimensional "shell" embedded in three-dimensional space, where the mirror symmetry $\mathcal{M}_{++}$of the two-dimensional model becomes a twofold rotation symmetry $\mathcal{R}_{++}$in three dimensions. When localized near the sample boundaries, the $\mathcal{M}$-breaking mass term $\mu_{2} \sigma_{1} \tau_{1}$ does not obstruct this deformation procedure, while ensuring that any helical boundary modes running along the two "seams" of the shell are gapped out apart from the two corners on the rotation axis; see Fig. 8 . The corners on the rotation axis each host a Kramers pair of zero-energy states.

We now discuss the consequences for the boundary classification of third-order topological phases in class $\mathrm{D}^{\mathcal{R}_{++}}$in three dimensions. Since the twofold rotation symmetry is a local symmetry at the two corners on the rotation axis, zero-energy corner states have an extrinsic $\mathcal{D}_{0}^{\prime \prime \prime}=\mathbb{Z}_{2}^{2}$ classification, the two $\mathbb{Z}_{2}$ topological indices counting the parities of the numbers of Kramers pairs of such corner states that are even or odd under $\mathcal{R}$, respectively. Kramers pairs of zero-energy corner states can be obtained by decoration with one-dimensional topological superconductors along crystal hinges, but such a procedure

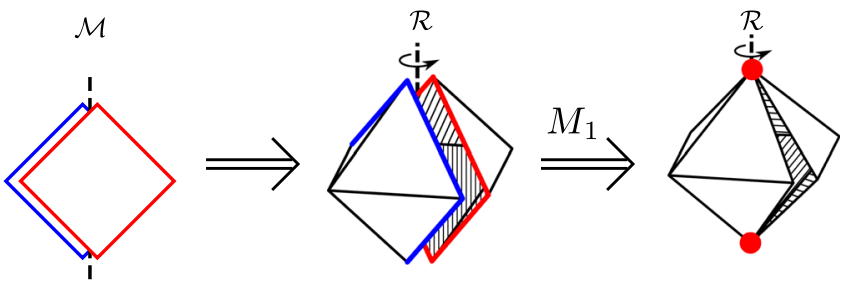

FIG. 8. Two copies of a topologically nontrivial superconductor in class DIII that are related by mirror symmetry $\mathcal{M}$ form a separable topological phase (left). The separable phase can be deformed into a hollow shell, such that the mirror symmetry $\mathcal{M}$ becomes a twofold rotation symmetry $\mathcal{R}$ (center). Upon addition of a mass term along the "seams" of the shell, while preserving the global twofold rotation symmetry, any pairs of counterpropagating Majorana hinge modes can be gapped out, leaving behind Kramers pairs of zero-energy states at the two corners on the rotation axis (right). 
always gives equal number of even-parity and odd-parity states. Hence, corner states obtained from decorations along hinges have classifying group $\mathcal{D}_{2}^{\prime \prime \prime}=\mathbb{Z}_{2}$, corresponding to the "diagonal" elements of $\mathcal{D}_{0}^{\prime \prime \prime}=\mathbb{Z}_{2}^{2}$. To obtain a single Kramers pair of zero-energy corner states one must decorate the crystal boundary with a two-dimensional shell as constructed above. As a result, one finds $\mathcal{D}_{1}^{\prime \prime \prime}(3,2)=$ $\mathcal{D}_{0}^{\prime \prime \prime}=\mathbb{Z}_{2}^{2}$. The resulting boundary classifying groups then follow by taking quotients, $\mathcal{K}_{a}^{\prime \prime \prime}(3,2)=\mathcal{K}_{1}^{\prime \prime \prime}(3,2)=0$, $\mathcal{K}_{2}^{\prime \prime \prime}(3,2)=\mathbb{Z}_{2}$; see Table XII.

Class $A^{\mathcal{P}^{+} \mathcal{M}}$ in two dimensions. - A separable secondorder phase in class $\mathrm{A}^{\mathcal{P}^{+} \mathcal{M}}$ can be constructed from two copies of a quantum Hall system related to each other by particle-hole conjugation. The minimal canonicalform Hamiltonian describing such a separable phase is specified by

$$
\Gamma_{0}=\tau_{3} \sigma_{1}, \quad \boldsymbol{\Gamma}=\left(\tau_{0} \sigma_{2}, \tau_{0} \sigma_{3}\right) .
$$

This Hamiltonian has an antiunitary mirror antisymmetry represented by $U_{\mathcal{P M}}=\tau_{1}$. It has two $\mathcal{P} \mathcal{M}$-breaking mass terms $M_{1}=\tau_{1} \sigma_{1}$ and $M_{2}=\tau_{2} \sigma_{1}$, which render it a secondorder topological phase with a single zero-energy state at mirror-symmetric corners. Proceeding as before, we can use this Hamiltonian to decorate a three-dimensional crystal, whereby the $\mathcal{P}^{+} \mathcal{M}$ mirror antisymmetry of the two-dimensional Hamiltonian turns into a $\mathcal{P}^{+} \mathcal{R}$ rotation antisymmetry in three dimensions. Again, the mass terms $M_{1}$ and $M_{2}$ do not obstruct the deformation procedure if they are localized near the seams of the shell only, while the presence of such mass terms ensures that the entire shell is gapped out, except for the corners at the twofold rotation axis, which host zero-energy states. From the boundary perspective, one has the extrinsic classifying group $\mathcal{D}_{0}^{\prime \prime \prime}=\mathbb{Z}_{2}$, which counts the parity of the number of such zero-energy corner states. One-dimensional decorations along hinges cannot result in any protected zero-energy corner states in this symmetry class. However, zero-energy corner states can be obtained from a two-dimensional shell-like decoration as described above. We conclude that $\mathcal{D}_{1}^{\prime \prime \prime}(3,2)=\mathbb{Z}_{2}$ and $\mathcal{D}_{2}^{\prime \prime \prime}(3,2)=0$, giving the boundary classifying groups $\mathcal{K}_{a}^{\prime \prime \prime}(3,2)=\mathcal{K}_{1}^{\prime \prime \prime}(3,2)=0$ and $\mathcal{K}_{2}^{\prime \prime \prime}(3,2)=\mathbb{Z}_{2}$; see Table XI.

\section{Stacking construction}

We now discuss three examples that compare the action of the order-raising homomorphism $\omega$ and the stacking homomorphism $\sigma$. The first example is the canonical-form Hamiltonian $H_{d}$ from Shiozaki-Sato class $\mathrm{D}^{\mathcal{O}_{-}}$with $d=0$

$$
H_{0}(m)=m \sigma_{1},
$$

with $U_{\mathcal{O}}=\sigma_{1}$ and $U_{\mathcal{P}}=\sigma_{3}$. (Keeping the dependence on the parameter $m$ is necessary to allow for a meaningful distinction between topological phases in zero dimensions. For zero-dimensional Hamiltonians, one can uniquely assign topological invariants only to the one-parameter family of Hamiltonians, but not to the Hamiltonian itself; see the discussion in Sec. II.) The stacking procedure gives a one-dimensional Hamiltonian $\sigma\left[H_{0}(m)\right]$ in class $\mathrm{D}^{\mathcal{M}_{-}}$. The upper-left block $H_{1}^{\prime}$ of Eq. (38) takes the form

$$
H_{1}^{\prime}(k)=m\left(\sigma_{1} \cos k+\sigma_{2} \sin k\right),
$$

where we take $\hat{\rho}=\sigma_{3}$ in Eq. (38). The lower-right block of the Hamiltonian $H_{1}$ of Eq. (38) is $k$ independent and it does not carry any strong topological invariants. Since the above Hamiltonian is not in canonical form, we calculate the topological invariant $N=n_{o}(\pi)-n_{o}(0)$ for the Hamiltonian $H_{1}^{\prime}\left(k_{1}\right)$, where $n_{o}(k)$ is the number of the odd-parity negative-energy eigenvalues at the inversionsymmetric momentum $k=0, \pi$. We find that $H_{1}^{\prime}\left(k_{1}\right)$ has $N=1$ for $m<0$ and $N=-1$ for $m>0$, therefore the oneparameter family (50) has topological invariant $N=2$. The same is true for $\omega\left(H_{0}\right)$, as one verifies using the explicit representation of $\omega$ given in Table XV.

For the second example, we consider a canonical-form Hamiltonian $H_{d}$ from Shiozaki-Sato class $\mathrm{D}^{\mathcal{M}_{-}}$with $d=1$, specified by

$$
\Gamma_{0}=\sigma_{1}, \quad \boldsymbol{\Gamma}=\left(\sigma_{2}\right),
$$

with $U_{\mathcal{M}}=\sigma_{1}$ and $U_{\mathcal{P}}=\sigma_{3}$. The above Hamiltonian describes a one-dimensional $p$-wave superconductor with a single Majorana mode localized at each end. The application of the stacking construction to the one-dimensional superconductor with Hamiltonian $H_{d}$ specified by matrices (51) gives the Hamiltonian $\sigma\left(H_{d}\right)$ with $d=1$, and the upper-right block $H_{d+1}^{\prime}$

$$
\begin{aligned}
H_{2}^{\prime}= & \left(m+1-\cos k_{1}\right)\left(\sigma_{1} \cos k_{2}-\sigma_{3} \sin k_{2}\right) \\
& +\sin k_{1} \sigma_{2},
\end{aligned}
$$

where we used $\hat{\rho}=\sigma_{2}$, compare with Eq. (38). Since this Hamiltonian is not of minimal canonical form, its topological invariant cannot simply be determined by counting the number of bands. The topological invariant $N$ in this class takes integer values [6,21]

$$
N=n_{o}(\pi, \pi)-n_{o}(\pi, 0)-n_{o}(0, \pi)+n_{o}(0,0),
$$

where $n_{o}(\boldsymbol{k})$ counts the number of odd-parity negative eigenvalues at the high-symmetry momentum $\boldsymbol{k}=\left(k_{1}, k_{2}\right)$. Direct calculation gives that both $\sigma\left(H_{d}\right)$ and $\omega\left(H_{d}\right)$ have $N=2$ for $d=2$.

Finally, we apply the stacking homomorphism $\sigma$ to a first-order nonseparable superconductor in class $\mathrm{D}^{\mathcal{R}_{-}}$, with two-dimensional Hamiltonian specified by 


$$
\Gamma_{0}=\sigma_{1}, \quad \boldsymbol{\Gamma}=\left(\sigma_{2}, \sigma_{3}\right),
$$

with $U_{\mathcal{R}}=\sigma_{1}$ and $U_{\mathcal{P}}=\sigma_{3}$. We choose $\hat{\rho}=\sigma_{2}$ and obtain the upper-left block $H_{d+1}^{\prime}$ of Eq. (38) as

$$
\begin{aligned}
H_{3}^{\prime}\left(k_{1}, k_{2}, k_{3}\right)= & \left(m+2-\cos k_{1}\right)\left(\sigma_{1} \cos k_{3}-\sigma_{3} \sin k_{3}\right) \\
& +\sigma_{2} \sin k_{1}-\sigma_{1} \cos \left(k_{1}+k_{3}\right) \\
& +\sigma_{3} \sin \left(k_{1}+k_{3}\right)
\end{aligned}
$$

which has inversion symmetry with $U_{\mathcal{I}}=\sigma_{1}$, and particlehole antisymmetry $U_{\mathcal{P}}=\sigma_{3}$. For class $\mathrm{D}^{\mathcal{I}_{-}}$in three dimensions, similar to the previously considered classes, the topological invariant $N$ can be evaluated via the inversion eigenvalues of the occupied bands $[6,21]$

$$
\begin{aligned}
N= & {\left[n_{o}(\pi, \pi, \pi)-n_{o}(\pi, \pi, 0)-n_{o}(\pi, 0, \pi)\right.} \\
& -n_{o}(0, \pi, \pi)+n_{o}(\pi, 0,0)+n_{o}(0, \pi, 0) \\
& \left.+n_{o}(0,0, \pi)-n_{o}(0,0,0)\right] / 2,
\end{aligned}
$$

We find that both $\omega\left(H_{d}\right)$ and $\sigma\left(H_{d}\right)$ have $N=1$ for $d=2$, accordingly they are deformable into each other.

\section{Embedded topological phases}

It was pointed out recently [65] that in the presence of crystalline symmetries a lower-dimensional topological phase embedded in a higher-dimensional topologically trivial bulk-a so-called "embedded topological phase"has the same boundary phenomenology as the higher-order topological phases considered in this work. Can an embedded topological phase with Hamiltonian $H$ be deformed into a higher-order topological crystalline phase with Hamiltonian $\omega(H)$ ? The same question was recently addressed by Matsugatani and Watanabe using a slightly different approach [56].

Figure 9(a) shows that the stacked-layer system $\sigma(H)$ can be deformed to the corresponding embedded topological system by breaking the crystalline symmetry $\mathcal{S}$ locally by dimerizing the layers, while globally preserving $\mathcal{S}$ symmetry. Using the conclusions of the previous section we obtain that $\omega(H) \cong \sigma(H)$ is deformable to the corresponding embedded system using a deformation that breaks $\mathcal{S}$ locally, while preserving it globally-below we arrive at the same conclusion using a different argument.

Assuming for concreteness that the Hamiltonian $\omega(H)$ is a three-dimensional inversion-symmetric, second-order Chern insulator with a single hinge mode at its boundary, Fig. 9(b) shows that its halves above and below the hinge mode can be trivialized as the local symmetry is broken, because $\omega(H)$ has only purely crystalline topological invariants. This construction immediately enables us to conclude that $\omega(H)$ is deformable to an embedded topological insulator.
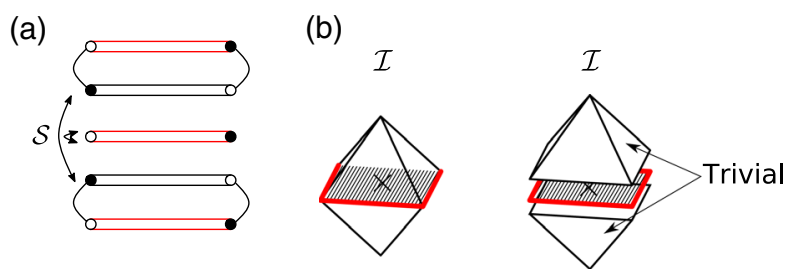

FIG. 9. (a) Dimerization of a stacked-layer system that locally breaks $\mathcal{S}$ (anti)symmetry, while preserving $\mathcal{S}$ globally. (b) After breaking the local inversion symmetry, the upper and the lower halves of three-dimensional second-order Chern insulator can be trivialized, resulting in a embedded topological insulator.

\section{ORDER-LOWERING MAP $\bar{\omega}$}

In this section, we introduce an order-lowering map $\bar{\omega}$ that acts on a canonical-form Hamiltonian $H$ with anomalous boundary states of order $n>1$ and gives a Hamiltonian $\bar{\omega}(H)$ such that $\omega[\bar{\omega}(H)]$ is continuously deformable to the original Hamiltonian $H$. Although the map $\bar{\omega}$ can be defined entirely algebraically, there is a simple geometric picture underlying the construction of $\bar{\omega}$, which we discuss first.

To explain the geometrical picture underlying the construction of the order-lowering map $\bar{\omega}$, we recall the introduction of the sequence of manifolds (19) in Sec. IV and the subsequent observation that an $n$ th-order topological phase with $n>1$ is essentially trivial away from $\Omega_{d-n+1} \subseteq \Omega_{d-1}$. For a Hamiltonian in canonical form we choose $\Omega_{d-1}$ to be the intersection of the crystal with the hyperplane $x_{1}=0$. Since $x_{1} \rightarrow-x_{1}$ under the crystalline (anti)symmetry $\mathcal{S}$, the hyperplane $x_{1}=0$ divides the crystal into two symmetry-related "halves." We smoothly deform the Hamiltonian by adding the term $m_{1} M_{i} \operatorname{sign}\left(x_{1}\right)$, where $M_{i}$ is a crystalline-symmetry-breaking mass term and $m_{1}>0$. Although this extra term locally breaks the crystalline (anti)symmetry $\mathcal{S}, \mathcal{S}$ is preserved globally. Taking the limit $m_{1} \rightarrow \infty$ amounts to a projection onto the hyperplane $x_{1}=0$, which gives the $(d-1)$ dimensional Hamiltonian

$$
\bar{\omega}\left(H, M_{i}\right)=P_{i} H P_{i},
$$

where $P_{i}=\left(i M_{i} \Gamma_{1}+1\right) / 2$ is a projection operator. The Hamiltonian $\bar{\omega}\left(H, M_{i}\right)$ obeys a crystalline (anti)symmetry with $d_{\|}-1$ inverted dimensions, which is obtained by restricting $\mathcal{S}$ to the plane $x_{1}=0$. If $H$ has anomalous boundary states of codimension $n$, so has $\bar{\omega}\left(H, M_{i}\right)$ anomalous boundary states of codimension $n-1$. (The inverse is not true; see the discussion below.) A variant of this construction was suggested by Matsugatani and Watanabe, who instead of adding a crystalline-symmetry-breaking mass term proposed to symmetrically remove the crystal on both sides of $\Omega_{d-1}$ [56].

We illustrate this procedure using the example of a threedimensional second-order Chern insulator with inversion 


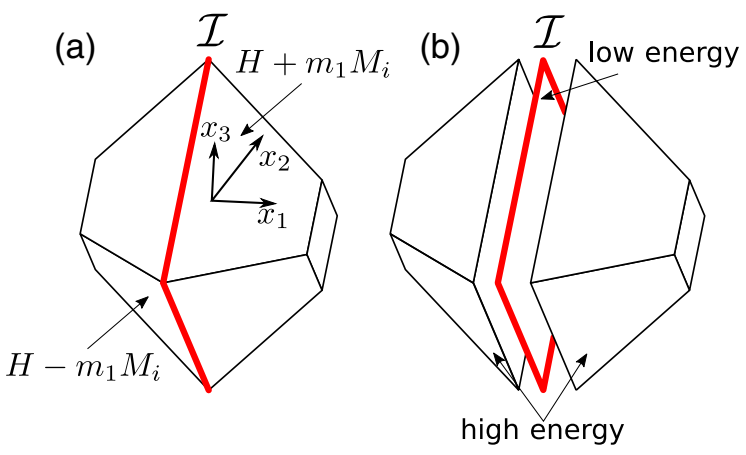

FIG. 10. A three-dimensional, inversion-symmetric, secondorder phase can be mapped onto a two-dimensional first-order phase. The map is achieved by adding a crystalline-symmetrybreaking mass term $M_{i}$ with opposite prefactors on both sides of a plane that symmetrically divides the crystal into two halves and that contains the anomalous boundary states (a). Such an additional mass term respects the inversion rotation symmetry and manifestly trivializes the three-dimensional bulk away from the boundary states. The low-energy theory consists of a first-order two-dimensional Hamiltonian (b).

symmetry $\mathcal{I}$, see Fig. 10. The canonical-form Hamiltonian for this topological phase is specified by

$$
\Gamma_{0}=\sigma_{2} \tau_{3}, \quad \boldsymbol{\Gamma}=\left(\sigma_{0} \tau_{1}, \sigma_{3} \tau_{3}, \sigma_{1} \tau_{3}\right),
$$

where we use the representation $U_{\mathcal{I}}=\tau_{3} \sigma_{2}$. There is one crystalline-symmetry-breaking mass term $M_{1}=\tau_{2}$. Adding a term $m_{1} M_{1} \operatorname{sign}\left(x_{1}\right)$ preserves the global inversion symmetry, while manifestly opening up a spectral gap away from the plane $x_{1}=0$. If the spectral gap from this additional mass term is much larger than the other spectral gaps, we may perform a partial low-energy expansion along the $x_{1}$ direction, which yields the three-dimensional Hamiltonian

$$
\begin{aligned}
H= & \left(m+2-\cos k_{1}-\cos k_{2}\right) \Gamma_{0}+\sin k_{2} \Gamma_{2}+\sin k_{3} \Gamma_{3} \\
& +m_{1} M_{1} \operatorname{sign}\left(x_{1}\right)-i \partial_{x_{1}} \Gamma_{1} .
\end{aligned}
$$

Taking $m_{1} \gg|m| \sim 1$, we obtain the two-dimensional effective Hamiltonian

$$
\begin{aligned}
H_{2}= & P_{1}\left[\left(m+2-\cos k_{1}-\cos k_{2}\right) \Gamma_{0}\right. \\
& \left.+\sin k_{2} \Gamma_{2}+\sin k_{3} \Gamma_{3}\right] P_{1},
\end{aligned}
$$

which describes the quantum Hall phase [compare with Eq. (39)].

The above construction of the inverse map $\bar{\omega}$ depends explicitly on the form of the crystalline-symmetry-breaking mass term $M_{i}$ and on the choice of the hyperplane $\Omega_{d-1}$. Indeed, in general there is no unique inverse map for the order-raising map $\omega$, because $\omega$ is a homomorphism, not an isomorphism. However, the order-raising homomorphism $\omega$ becomes an isomorphism when seen as a map between the quotient groups $K^{(n)}\left(d, d_{\|}\right) / \operatorname{ker} \omega$ and its image $K^{(n+1)}\left(d+1, d_{\|}+1\right)$. Regarded as a map between these two groups, the above-defined map $\bar{\omega}$ is a true inverse map that does not depend on the choice of $M_{i}$.

To illustrate the "nonuniqueness" of the orderlowering map, we consider the trivial inversion-symmetric Hamiltonian $H$ in three dimensions specified by

$$
\Gamma_{0}=\mu_{3} \tau_{0} \sigma_{1}, \quad \boldsymbol{\Gamma}=\left(\mu_{1}, \mu_{3} \tau_{0} \sigma_{2}, \mu_{3} \tau_{0} \sigma_{3}\right) .
$$

Inversion symmetry is represented using $U_{\mathcal{I}}=\mu_{3} \tau_{1} \sigma_{1}$. The above Hamiltonian has the crystalline-symmetrybreaking mass term $M_{1}=\mu_{2}$, as well as a symmetrypreserving mass term $M=\mu_{2} \tau_{3}$. A direct application of Eq. (57) gives that $\bar{\omega}\left(H, M_{1}\right)$ is a canonical-form Hamiltonian specified by

$$
\Gamma_{0}=\tau_{0} \sigma_{1}, \quad \boldsymbol{\Gamma}=\left(\tau_{0} \sigma_{2}, \tau_{0} \sigma_{3}\right),
$$

and with the twofold rotation symmetry $U_{\mathcal{R}}=\tau_{1} \sigma_{1}$. This Hamiltonian has two chiral boundary modes, implying that it corresponds to a nontrivial element of $K(2,2)$, despite the three-dimensional Hamiltonian $H$ being trivial (because of the existence of the mass term $M)$. However, $\bar{\omega}\left(H, M_{1}\right)$ is manifestly separable, so that it corresponds to the trivial element of the group $K(2,2) / \operatorname{ker} \omega$. On the other hand, a different choice of the crystalline-symmetry-breaking mass term, $M_{1}=\mu_{2} \tau_{1}$, gives a different inverse, $\bar{\omega}\left(H, \mu_{2} \tau_{1}\right)$, which is a topologically trivial member of $K(2,2)$ since there is a nonzero crystalline-symmetry-preserving mass term $P_{1} M P_{1}$.

\section{BULK CLASSIFYING GROUPS FROM BOUNDARY AND ATOMIC-LIMITS CLASSIFICATION}

The bulk-boundary correspondence (2), together with the observation that the classifying groups $K^{(d)}$ represent atomic-limit phases, can be used to calculate the subgroup series (1) of bulk classifying groups (see Tables II-VII) without using the $K$-theory-based classification of Shiozaki and Sato [21]. In this section we explain how such a calculation proceeds. The advantage of such an approach is that in principle it is not restricted for the order-two crystalline (anti)symmetries, for which the $K$-theory approach of Ref. [21] was derived.

The inputs for the calculation described below are the tenfold-way classifying groups in $d$ dimensions $K_{\mathrm{TF}}(d)$, their subgroups $K_{\mathrm{TF}, \mathcal{S}}\left(d, d_{\|}\right)$describing tenfold-way phases compatible with the crystalline symmetry $\mathcal{S}$, as well as the groups $K(d, 0)$ classifying tenfold-way phases with an additional on-site symmetry, all of which can be obtained by elementary means from the known tenfold-way classification. The construction below requires an explicit realization of the order-raising homomorphism $\omega$ and its inverse map $\bar{\omega}$, which can be done using the geometric construction of the previous section in the case of an ordertwo symmetry. 


\section{A. Calculation of $\boldsymbol{K}^{(d)}$}

The group $K^{(d)}$ is obtained from the Shiozaki-Sato group $K(0,0)$ that classifies topological zero-dimensional phases protected with an on-site (i.e., internal) symmetry. Following the discussion of Sec. IV, the calculation of $K^{(d)}$ amounts to the calculation of the subgroup ker $\omega^{d} \subseteq K(0,0)$. If $H_{0}$ is a generator of the corresponding zero-dimensional tenfold-way phase, the manifestly separable Hamiltonian $\operatorname{diag}\left(H_{0}, \mathcal{S} H_{0}\right)$ is the generator of ker $\omega$. Similarly, $H_{1}$, a generator of one-dimensional tenfold-way phase, defines a one-dimensional separable Hamiltonian $H_{1}^{\prime}=\left[H_{1}, \mathcal{S} H_{1}\right]$. If $H_{1}^{\prime}$ is topologically nontrivial (i.e., has no additional mass terms) and has at least one crystallinesymmetry-breaking mass term $M_{1}$, then the zero-dimensional Hamiltonian $\bar{\omega}\left(H_{1}^{\prime}, M_{1}\right)$ exists and is an element the group $K(0,0)$. The kernel $\operatorname{ker} \omega^{2}$ is the subgroup of $K(0,0)$ generated by $\operatorname{ker} \omega$ and $\bar{\omega}\left(H_{1}^{\prime}, M_{1}\right)$. (Note that the subgroup ker $\omega^{2}$ defined this way is uniquely defined, in spite of the nonuniqueness of $\bar{\omega}$.) This procedure can be continued until ker $\omega^{d}$ is obtained, which then gives $K^{(d)}=K(0,0) / \operatorname{ker} \omega^{d}$.

\section{B. Classification anomalous boundary of codimension $\boldsymbol{n}$}

The calculation of the anomalous boundary classification $\mathcal{K}_{a}^{(n)}$ starts from the extrinsic boundary classification group $\mathcal{D}_{0}^{(n)}$ and the decoration subgroups $\mathcal{D}_{k}^{(n)}$; see Sec. IV. For $n=d_{\|}+1$, the extrinsic classifying group $\mathcal{D}_{0}^{(n)}=$ $K\left(d-d_{\|}, 0\right)$ is the classification of $\left(d-d_{\|}\right)$-dimensional tenfold-way phases protected with an on-site (internal) symmetry. The decoration groups are subgroups of the extrinsic classifying group, $\mathcal{D}_{k}^{(n)}=\operatorname{ker} \omega^{n-k} \subseteq \mathcal{D}_{0}^{(n)}$. Their calculations proceed along the same line as in the calculation of $K^{(d)}$ discussed above.

To calculate $\mathcal{K}_{a}^{(n)}$ for $n \leq d_{\|}$, we need the classification of the tenfold-way phases $K_{\mathrm{TF}, \mathcal{S}}\left(d, d_{\|}\right) \subseteq K_{\mathrm{TF}}(d)$ that are compatible with the crystalline (anti)symmetry $\mathcal{S}$. To this end, we notice that a canonical-form tenfold-way Hamiltonian $H$ has the most symmetric form-its lowenergy expansion has the full rotational symmetry, with generators that are pairwise products of the kinetic terms. Accordingly, it is sufficient to check if a canonical-form Hamiltonian $H$ is compatible with the (anti)symmetry $\mathcal{S}$, which is a matter of algebra. Once the extrinsic boundary classification group $\mathcal{D}_{0}^{(n)}$ is known, the decoration group $\mathcal{D}_{n-1}^{(n)}$ is generated by the Hamiltonian $\operatorname{diag}\left(H_{d+1-n}\right.$, $\left.\mathcal{S} H_{d+1-n}\right)$, where $H_{d+1-n}$ is a generator Hamiltonian of $(d+1-n)$-dimensional tenfold-way phase without additional crystalline symmetry. Furthermore, if the Hamiltonian $H_{d+2-n}^{\prime}=\operatorname{diag}\left(H_{d+2-n}, \mathcal{S} H_{d+2-n}\right)$ is topologically nontrivial and has one crystalline-symmetry-breaking mass term $M_{1}$, then the Hamiltonian $\bar{\omega}\left(H_{d+2-n}, M_{1}\right)$ is a $(d+1-n)$-dimensional Hamiltonian representing an element of $K_{\mathrm{TF}, \mathcal{S}}$. In this way we obtain $\mathcal{D}_{n-2}^{(n)}$ as the subgroup spanned by $\mathcal{D}_{n-1}^{(n)}$ and $\bar{\omega}\left(H_{d+2-n}, M_{1}\right)$. This procedure is repeated until the group $\mathcal{D}_{1}^{(n)}$ is reached.

\section{Calculating $K^{(n)}$ from the groups $K^{(d)}$ and $\mathcal{K}_{a}^{(k)}$}

The bulk-boundary correspondence (2) can be rewritten in form of an exact sequence

$$
0 \rightarrow K^{(n+1)} \rightarrow K^{(n)} \rightarrow \mathcal{K}_{a}^{(n+1)} \rightarrow 0, \quad n=0,1, \ldots, d .
$$

If the boundary classification group $\mathcal{K}_{a}^{(n+1)}$ is a free Abelian group (i.e., it is of the form $\mathbb{Z}^{k}$ ), then the above exact sequence splits and has the unique solution $K^{(n)}=$ $K^{(n+1)} \oplus \mathcal{K}_{a}^{(n+1)}$. On the other hand, if the boundary classification has a torsion subgroup (e.g., it contains $\mathbb{Z}_{2}$ ) then the above exact sequence has in general more than one solution and knowledge of the groups $\mathcal{K}_{a}^{(n+1)}$ and $K^{(n+1)}$ is not sufficient to determine $K^{(n)}$. Such an extension problem can be formulated as an algebraic problem: Assume $g$ is a torsion element from $\mathcal{K}_{a}^{(n+1)}$, i.e., $\oplus^{k} g=e$ is the trivial element for some $k$. (For the order-two symmetries one always has $k=2$.) Let $H$ be a bulk Hamiltonian that generates the state $g$ on its boundary. The Hamiltonian $H$ represents an element of $K^{(n)}$, but not of $K^{(n+1)}$. The $k$-fold direct sum $\oplus^{k} H$ is either trivial or it is a generator of the subgroup $K^{(n+1)} \subseteq K^{(n)}$. With this additional knowledge, which can be determined by checking for additional mass terms of the Hamiltonian $\oplus^{k} H$, the group $K^{(n)}$ can be uniquely determined from $K^{(n+1)}$ and $\mathcal{K}_{a}^{(n+1)}$.

\section{Example: Classification of inversion-symmetric 3D topological insulator in class A}

As an example, we now show how the above procedure can be used to obtain the full classifying subgroup sequence of an inversion-symmetric topological insulator in three dimensions. We start with the group $K^{(d)}$ classifying atomic-limit insulators. To obtain $K^{(d)}$, we need the classification $K(0,0)$ of zero-dimensional Hamiltonians protected by an on-site symmetry $\mathcal{O}$. Such zerodimensional Hamiltonians can be block diagonalized where each block has the classification of the zero-dimensional Hamiltonian of symmetry class A,

$$
K(0,0)=\left\{\left(n_{1}, n_{2}\right), n_{1}, n_{2} \in \mathbb{Z}\right\}=\mathbb{Z}^{2},
$$

where the integers $n_{1}$ and $n_{2}$ count the numbers of occupied states of even and odd parity, respectively. Furthermore, taking $H_{0}=\sigma_{3}$ as a generator of the tenfold-way class A, the separable Hamiltonian $\operatorname{diag}\left(H_{0}, H_{0}\right)=\tau_{0} \sigma_{3}$ has on-site symmetry $U_{\mathcal{O}}=\tau_{1}$ and has topological number $(1,1)$, so that 


$$
\operatorname{ker} \omega=\{(n, n), n \in \mathbb{Z}\}=\mathbb{Z} .
$$

Further, since there are no topologically nontrivial onedimensional tenfold-way phases in A, it follows that ker $\omega^{2}=\operatorname{ker} \omega$, and since the separable nontrivial twodimensional phase is a first-order phase (it has two chiral modes on its boundary) one even has ker $\omega^{3}=\operatorname{ker} \omega$. In this way, we arrive at the classification of atomic limits

$$
K^{\prime \prime \prime}(3,3)=K(0,0) / \operatorname{ker} \omega^{3}=\mathbb{Z} .
$$

For the classification of anomalous boundary states, we can immediately conclude that the groups $\mathcal{K}_{a}^{\prime \prime \prime}$ and $\mathcal{K}_{a}^{\prime}$ are trivial since there are no topologically nontrivial tenfoldway phases in class $\mathrm{A}$ in one and three dimensions and since inversion leaves no points on the boundary invariant. To obtain the second-order boundary classification group $\mathcal{K}_{a}^{\prime \prime}$ we first need to check if a (two-dimensional) Chern insulator is compatible with twofold rotation symmetry. This is indeed the case, as demonstrated by the canonicalform Hamiltonian

$$
\Gamma_{0}=\sigma_{1}, \quad \boldsymbol{\Gamma}=\left(\sigma_{2}, \sigma_{3}\right),
$$

which is compatible with the twofold rotation symmetry represented by $U_{\mathcal{R}}=\sigma_{1}$. Thus,

$$
\mathcal{D}_{0}^{\prime \prime}=K_{\mathrm{TF}, \mathcal{R}}(2,2)=\mathbb{Z} \text {. }
$$

Moreover, since the diagonal sum $\operatorname{diag}\left(\mathrm{H}_{2}, \mathcal{R H}_{2}\right)$ has a Chern number equal to two (i.e., it has two copropagating chiral boundary modes), one finds

$$
\mathcal{D}_{1}^{\prime \prime}=2 \mathbb{Z} \subseteq K_{\mathrm{TF}}(2,2),
$$

so that

$$
\mathcal{K}_{a}^{\prime \prime}=\mathbb{Z}_{2}
$$

To obtain the bulk subgroup sequence (1) from the classification results $K^{\prime \prime \prime}(3,3)=\mathbb{Z}, \mathcal{K}_{a}^{\prime \prime \prime}=0, \mathcal{K}_{a}^{\prime \prime}=\mathbb{Z}_{2}$, and $\mathcal{K}_{a}^{\prime}=0$, we need to solve the exact sequence (63) to obtain $K^{\prime \prime}(3,3), K^{\prime}(3,3)$, and $K(3,3)$. Here, only the case $n=1$ is nontrivial,

$$
0 \rightarrow \mathbb{Z} \rightarrow K^{\prime}(3,3) \rightarrow \mathbb{Z}_{2} \rightarrow 0 .
$$

In order to resolve the above sequence we need to know if the sum of two second-order phases yields a trivial phase, in which case one has $K^{\prime}(3,3)=\mathbb{Z}_{2} \oplus \mathbb{Z}$, or if it yields a topologically nontrivial atomic insulator, in which case the bulk classification is $K^{\prime}(3,3)=\mathbb{Z}$. We answer this question by considering the direct sum of two second-order phases. Such a direct sum is given by the sum of two second-order Chern insulators with copropagating chiral modes along hinges. Following the procedure outlined in Sec. VII, these three-dimensional inversion-symmetric second-order Chern insulators can be seen as two-dimensional (first-order) Chern insulators embedded in an otherwise trivial three-dimensional crystal. By rotating one of the two embedded Chern insulators with respect to the other, the system may be deformed such that the two chiral hinge modes are counterpropagating, corresponding to the two-dimensional Hamiltonian

$$
\Gamma_{0}=\tau_{0} \sigma_{1}, \quad \boldsymbol{\Gamma}=\left(\tau_{0} \sigma_{2}, \tau_{3} \sigma_{3}\right) .
$$

The inversion symmetry of the three-dimensional host crystal becomes a twofold rotation symmetry for the twodimensional Chern insulator, represented by $U_{\mathcal{R}}=\tau_{0} \sigma_{1}$. The above Hamiltonian has two mass terms $\tau_{1} \sigma_{3}$ and $\tau_{2} \sigma_{3}$ which break the twofold rotation symmetry $\mathcal{R}$. We therefore conclude that the resulting Hamiltonian is a topologically nontrivial atomic limit, so that $K^{\prime}(3,3)=\mathbb{Z}$. The resulting bulk subgroup sequence then reads

$$
2 \mathbb{Z} \subseteq 2 \mathbb{Z} \subseteq \mathbb{Z} \subseteq \mathbb{Z},
$$

which agrees with the corresponding entry in Table VII. (Note that although we originally identified $K^{\prime \prime \prime}$ with $\mathbb{Z}$, this identification must be reconsidered in view of the fact that $K^{\prime \prime \prime}=K^{\prime \prime}$ is a subgroup of $K^{\prime}=K$ and that $K^{\prime} / K^{\prime \prime}=\mathbb{Z}_{2}$.)

\section{CONCLUSIONS}

Topological crystalline insulators and superconductors have a more subtle boundary signature of a nontrivial bulk topology than topological phases that do not rely on the protection by a crystalline symmetry. Whereas the latter have a bulk-boundary correspondence involving the crystal's full boundary, such that a nontrivial topology is uniquely associated by a gapless boundary state, topological crystalline insulators or superconductors may also have protected gapless boundary states of codimension larger than one or they may have no boundary signatures at all. In this work, we provide the formal framework for a classification of topological crystalline phases that fully accounts for these different scenarios and provide such a classification for topological crystalline phases with an order-two crystalline symmetry or antisymmetry. This classification of bulk crystalline phases consists of a subgroup sequence $K^{(d)} \subseteq K^{(d-1)} \subseteq \cdots \subseteq K$, where the subgroup $K^{(n)}$ classifies bulk phases with boundary states of codimension larger than $n$. The first group in the sequence, $K^{(d)}$ classifies those bulk phases for which no boundary signature exists. Our classification identifies such phases as $d$-dimensional "stacks" of disconnected (zero-dimensional) objects, i.e., as an "atomic-limit" insulator. We contrast the subgroup sequence describing the bulk topology with a classification of codimension- $n$ boundary states. After dividing out codimension- $n$ boundary states which can also be obtained as boundary states of topological phases residing on the boundary-i.e., after dividing out boundary states that can be fully attributed to the crystal's termination-the resulting anomalous boundary classifying group $\mathcal{K}_{a}^{(n)}=K^{(n-1)} /$ $K^{(n)}$. This is the bulk-boundary correspondence for topological crystalline insulators. 
A central role in our construction is played by an orderraising homomorphism, which simultaneously raises the dimensionality $d$ of the Hamiltonian, the number of inverted dimensions $d_{\|}$of the order-two crystalline symmetry or antisymmetry, and the codimension $n$ of the boundary states (if any). For order-two symmetries, we find that the layerstacking construction used in Refs. [48,53,54,63] is a realization of the order-raising homomorphism. This is an important observation, since we found the explicit expression for the order-raising homomorphism $\omega$ only for ordertwo crystalline (anti)symmetries, whereas the layer-stacking construction can be applied to arbitrary crystalline (anti) symmetry, which makes it a valuable tool in obtaining the anomalous boundary classification of higher-order topological phases [48]. Finding anomalous boundary classifying groups is a simpler task [20] compared to finding the bulk classifying groups [23].

Our algebraic approach allowed us to obtain a full classification of higher-order phases of topological crystalline phases with an order-two crystalline symmetry without having to analyze each symmetry class in detail. This "efficiency" of the method also has a disadvantage, as it does not provide explicit expressions for topological invariants. Nevertheless, since our approach allows one to construct canonical-form Hamiltonians for the generators of the bulk classifying groups, the combined knowledge of the full classification and of the generators can be used to estimate to what extent topology can be described by "proxies," such as the symmetry-based indicators of Refs. [18,66]. (For example, a single $\mathbb{Z}_{2}$ indicator will provide a full description of a bulk topology if the classifying group is $\mathbb{Z}_{2}$, but not if it is $\mathbb{Z}_{2}^{2}$ or $\mathbb{Z}$.) Examples of such a procedure are given in Sec. VI. The relation of our algebraic approach to other classification approaches, such as the momentum space AtiyahHirzebruch spectral sequence [24] is still an open question.

The first element in the group sequence, $K^{(d)}$, is zero for crystalline (anti)symmetries with $d_{\|}<d$. These include mirror (anti)symmetry in dimensions $d \geq 2$ and twofold rotation (anti)symmetry in dimensions $d \geq 3$. On the other hand, for mirror symmetry with $d=1$, twofold rotation symmetry with $d=2$, and inversion symmetry with $d=3$, $K^{(d)}$ may be nonzero. A nonzero $K^{(d)}$ indicates that there topological phases with a nontrivial bulk topology but without topologically protected boundary states. In some cases, such topologically nontrivial phases without protected boundary states are characterized by other observable signatures, such as the presence of boundary charges (not states) [51,67], or quantized electric [32,33,68-70] or magnetic moments. Such signatures of a nontrivial bulk topology are not part of the higher-order bulk-boundary correspondence that we establish here, and it is an interesting open problem how they can be incorporated.

We hope the results of this work not only bear theoretical relevance, but will also help experimental efforts [71-73] to observe some of the rich boundary phenomenology of crystalline topological insulators and superconductors in solid-state systems. Currently the list of candidate materials for a second-order topological insulators consists of tintelluride [28], bismuth [44], magnetically doped bismuth selenide [74] and certain transition metal dichalcogenides [75]. Our complete classification may facilitate the search for other material candidates. Finally, we note that in this work only strong crystalline invariants were considered. We leave it for future works the study of HOTPs originating from weak crystalline topological invariants [76], which would further expand the list of potential solid-state material candidates.

\section{ACKNOWLEDGMENTS}

We thank Andrei Bernevig, Akira Furusaki, Max Geier, Eslam Khalaf, Felix von Oppen, Ken Shiozaki, Simon Trebst, and Haruki Watanabe for stimulating discussions. We acknowledge support by project A03 of the CRC-TR 183 and by the priority programme SPP 1666 of the German Science Foundation (DFG).

\section{APPENDIX A: BULK-BOUNDARY CORRESPONDENCE}

In this Appendix we show that a bulk-boundary correspondence for general topological crystalline phases follows immediately from the following statement, which has been proven for the phases with an order-two symmetry $\mathcal{S}$ in the main text: The topological classification of atomic limits $K_{A}$ is the same as the topological classification of the bulk phases with no boundary states $K^{(d)}$.

From the above statement, it follows that every topologically nontrivial nonatomic-limit bulk, classified by $K / K^{(d)}$, needs to have anomalous boundary states of a certain codimension. Thus, to prove the bulk-boundary correspondence we need to show that for every anomalous boundary state of codimension $n$ there is a bulk that is generating such boundary state. The existence of such bulk readily follows since each boundary state of given codimension can be generated by embedding a tenfold-way bulk phase (assuming bulk-boundary correspondence for the tenfold-way phases) in a topologically trivial bulk. (The stacking map $\sigma$ of Sec. V C or the order-raising homomorphism $\omega$ of Sec. VA are nothing but implementations of such an embedding procedure.)

To prove the statement that $K_{A}=K^{(d)}$ for an arbitrary symmetry group, it is enough to show that $K^{(d)} \subseteq K_{A}$, since atomic limits have no boundary states, so that automatically $K_{A} \subseteq K^{(d)}$. Since a bulk Hamiltonian with topological invariants from $K^{(d)}$ has no boundary states, we can perform the cutting procedure of Sec. VII of the main text, to reduce the system to a phase that consists of a zero-dimensional topologically nontrivial Hamiltonian 
embedded in a topologically trivial bulk-clearly, an atomic-limit phase, thus $K_{A}=K^{(d)}$.

\section{APPENDIX B: DIMENSION-RAISING ISOMORPHISMS}

The construction of the order-raising homomorphism $\omega$ requires us to include "defect Hamiltonians" $H(\boldsymbol{k}, \boldsymbol{\varphi})$ into our classification. Defect Hamiltonians were introduced for the tenfold-way classes by Teo and Kane [52], and considered for crystalline topological phases with an order-two symmetry or antisymmetry by Shiozaki and Sato [21]. Defect Hamiltonians with a one-dimensional defect variable $\varphi$ appear in the algebraic construction of the order-raising map; see Sec. VA. Following Ref. [21], in this section we introduce defect Hamiltonians of canonical form in a slightly more general setting, using defect variables of arbitrary dimension $\boldsymbol{\varphi}$. We proceed with a discussion of the associated dimension-raising isomorphisms $\kappa_{\|}, \kappa_{\perp}, \rho_{\|}$, and $\rho_{\perp}$, as well as the boundary map $\delta$.

Defect Hamiltonians.-We consider families of Hamiltonians $H(\boldsymbol{k}, \boldsymbol{\varphi}, m)$, where the $D$-dimensional "defect coordinate" $\boldsymbol{\varphi}=\left(\boldsymbol{\varphi}_{\|}, \boldsymbol{\varphi}_{\perp}\right)$ is defined on a torus [77]. Denoting the number of "inverted" defect coordinates as $D_{\|}$, the family of Hamiltonians $H(\boldsymbol{k}, \boldsymbol{\varphi}, m)$ transforms under unitary order-two (anti)symmetry $\mathcal{S}$ as

$$
\begin{aligned}
H(\boldsymbol{k}, \boldsymbol{\varphi}, m) & =\mathcal{S} H(\boldsymbol{k}, \boldsymbol{\varphi}, m) \\
& \equiv \sigma_{\mathcal{S}} U_{\mathcal{S}} H(\mathcal{S} \boldsymbol{k}, \mathcal{S} \boldsymbol{\varphi}, m) U_{\mathcal{S}}^{-1}, \\
\mathcal{S} \boldsymbol{k} & =\left(-\boldsymbol{k}_{\|}, \boldsymbol{k}_{\perp}\right), \mathcal{S} \boldsymbol{\varphi}=\left(-\boldsymbol{\varphi}_{\|}, \boldsymbol{\varphi}_{\perp}\right),
\end{aligned}
$$

where $\quad \boldsymbol{k}_{\|}=\left(k_{1}, \ldots, k_{d_{\|}}\right), \quad \boldsymbol{k}_{\perp}=\left(k_{d_{\|}+1}, \ldots, k_{d}\right), \quad \boldsymbol{\varphi}_{\|}=$ $\left(\varphi_{1}, \ldots, \varphi_{D_{\|}}\right), \boldsymbol{\varphi}_{\perp}=\left(\varphi_{D_{\|}+1}, \ldots, \varphi_{D}\right)$, and we use the notation of Sec. II. Similarly, antiunitary symmetry and antisymmetry operations are represented by unitary matrices $U_{\mathcal{S}}$,

$$
\begin{aligned}
H(\boldsymbol{k}, \boldsymbol{\varphi}, m) & =\mathcal{S} H(\boldsymbol{k}, \boldsymbol{\varphi}, m) \\
& \equiv \sigma_{\mathcal{S}} U_{\mathcal{S}} H^{*}(-\mathcal{S} \boldsymbol{k}, \mathcal{S} \boldsymbol{\varphi}, m) U_{\mathcal{S}}^{-1} .
\end{aligned}
$$

Dimension-raising isomorphisms. - The dimension-raising isomorphisms $\kappa_{\|}$and $\kappa_{\perp}$, which increase the dimension $d$ by one, were introduced in the main text. For defect Hamiltonians, two additional dimension-raising isomorphisms can be defined: The isomorphism $\rho_{\|}$, which increases by one both the defect dimension $D$ and the number of inverted defect coordinates $D_{\|}$, and the map $\rho_{\perp}$, which changes only the defect dimension $D$, such that [21]

$$
\begin{aligned}
K\left(s, t \mid d, d_{\|}, D, D_{\|}\right) & \stackrel{\rho_{\|}}{=} K\left(s-1, t-1 \mid d, d_{\|}, D+1, D_{\|}+1\right), \\
& \stackrel{\rho_{\perp}}{=} K\left(s-1, t \mid d, d_{\|}, D+1, D_{\|}\right), \quad \text { (B3) }
\end{aligned}
$$

for complex and real tenfold-way classes with a crystalline unitary order-two (anti)symmetry, and

$$
\begin{aligned}
K\left(s \mid d, d_{\|}, D, D_{\|}\right) & \stackrel{\rho_{\|}}{=} K\left(s+1 \mid d, d_{\|}, D+1, D_{\|}+1\right) \\
& \stackrel{\rho_{\perp}}{=} K\left(s-1 \mid d, d_{\|}, D+1, D_{\|}\right),
\end{aligned}
$$

for complex tenfold-way classes with a crystalline antiunitary order-two (anti)symmetry.

The action of these isomorphisms is defined [22,58] analogously to Eq. (36),

$$
\kappa[H(\boldsymbol{k}, \boldsymbol{\varphi}, m)]=H_{\kappa}\left(\boldsymbol{k}, \boldsymbol{\varphi}, m+1-\cos k^{\prime}\right)+\Gamma_{\kappa} \sin k^{\prime} .
$$

$\rho[H(\boldsymbol{k}, \boldsymbol{\varphi}, m)]=H_{\rho}\left(\boldsymbol{k}, \boldsymbol{\varphi}, m+1-\cos \varphi^{\prime}\right)+\Gamma_{\rho} \sin \varphi^{\prime}$.

If the defect coordinate $\varphi^{\prime}$ is flipped under the resulting crystalline symmetry, then the $(d+1)$-dimensional defect coordinate takes the form $\left(\varphi^{\prime}, \boldsymbol{\varphi}\right)$, otherwise it is $\left(\boldsymbol{\varphi}, \varphi^{\prime}\right)$. The form of the mapped Hamiltonian is listed in Tables XVI-XIX. The additional unitary (anti)symmetry $\mathcal{S}$ transforms as summarized in Tables XXI and XX.

TABLE XVI. The mapped Hamiltonian (B6) and the representation of the chiral symmetry $\mathcal{C}$ under application of the dimension-raising isomorphism $\rho$ for the complex tenfold-way classes.

\begin{tabular}{lcc}
\hline \hline TF class & $\left(H_{\rho}, \Gamma_{\rho}\right)$ & $\rho\left(U_{\mathcal{C}}\right)$ \\
\hline A & $\left(\tau_{3} H, \tau_{2}\right)$ & $\tau_{1}$ \\
AIII & $\left(H, U_{\mathcal{C}}\right)$ & $\cdots$ \\
\hline \hline
\end{tabular}

TABLE XVII. The mapped Hamiltonian (B6) and the representation of the antiunitary (anti)symmetries $\mathcal{T}$ and $\mathcal{P}$ under application of the dimension-raising isomorphism $\rho$ for the real tenfold-way classes.

\begin{tabular}{lccc}
\hline \hline TF classes & $\left(H_{\rho}, \Gamma_{\rho}\right)$ & $\rho\left(U_{\mathcal{T}}\right)$ & $\rho\left(U_{\mathcal{P}}\right)$ \\
\hline AI, AII & $\left(\tau_{3} H, \tau_{2}\right)$ & $\tau_{3} U_{\mathcal{T}}$ & $\tau_{2} U_{\mathcal{T}}$ \\
BDI, CII & $\left(H, U_{\mathcal{C}}\right)$ & $U_{\mathcal{T}}$ & $\cdots$ \\
D, C & $\left(\tau_{3} H, \tau_{2}\right)$ & $\tau_{1} U_{\mathcal{P}}$ & $\tau_{0} U_{\mathcal{P}}$ \\
DIII, CI & $\left(H, U_{\mathcal{C}}\right)$ & $\cdots$ & $U_{\mathcal{P}}$ \\
\hline \hline
\end{tabular}

TABLE XVIII. The mapped Hamiltonian (B5) and the representation of the chiral symmetry $\mathcal{C}$ under application of the dimension-raising isomorphism $\kappa$ for the complex tenfold-way classes.

\begin{tabular}{lcc}
\hline \hline TF class & $\left(H_{\kappa}, \Gamma_{\kappa}\right)$ & $\kappa\left(U_{\mathcal{C}}\right)$ \\
\hline A & $\left(\tau_{3} H, \tau_{2}\right)$ & $\tau_{1}$ \\
AIII & $\left(H, U_{\mathcal{C}}\right)$ & $\cdots$ \\
\hline \hline
\end{tabular}


TABLE XIX. The mapped Hamiltonian (B5) and the representation the antiunitary (anti)symmetries $\mathcal{T}$ and $\mathcal{P}$ under application of the dimension-raising isomorphism $\kappa$ for the real tenfold-way classes.

\begin{tabular}{lccc}
\hline \hline TF classes & $\left(H_{\kappa}, \Gamma_{\kappa}\right)$ & $\kappa\left(U_{\mathcal{T}}\right)$ & $\kappa\left(U_{\mathcal{P}}\right)$ \\
\hline AI, AII & $\left(\tau_{3} H, \tau_{2}\right)$ & $\tau_{0} U_{\mathcal{T}}$ & $\tau_{1} U_{\mathcal{T}}$ \\
BDI, CII & $\left(H, U_{\mathcal{C}}\right)$ & $\cdots$ & $U_{\mathcal{P}}$ \\
D, C & $\left(\tau_{3} H, \tau_{2}\right)$ & $\tau_{2} U_{\mathcal{P}}$ & $\tau_{3} U_{\mathcal{P}}$ \\
DIII, CI & $\left(H, U_{\mathcal{C}}\right)$ & $U_{\mathcal{T}}$ & $\cdots$ \\
\hline \hline
\end{tabular}

TABLE XX. The mapped representation of the unitary ordertwo (anti)symmetry $\mathcal{S}$ under application of the dimension-raising isomorphisms $\kappa_{\|}, \kappa_{\perp}, \rho_{\|}$, and $\rho_{\perp}$ for the complex tenfold-way classes. The mapping of the Hamiltonian and the tenfold-way symmetries is given in Table XVIII.

\begin{tabular}{lccc}
\hline \hline TF classes & $\mathcal{S}$ symmetry & $\kappa_{\|}, \rho_{\|}\left(U_{\mathcal{S}}\right)$ & $\kappa_{\perp}, \rho_{\perp}\left(U_{\mathcal{S}}\right)$ \\
\hline A & $\mathcal{S}$ & $\tau_{3} U_{\mathcal{S}}$ & $\tau_{0} U_{\mathcal{S}}$ \\
AIII & $\mathcal{S}_{+}$ & $U_{\mathcal{C}} U_{\mathcal{S}}$ & $U_{\mathcal{S}}$ \\
A & $\mathcal{C} \mathcal{S}$ & $\tau_{1} U_{\mathcal{S}}$ & $\tau_{2} U_{\mathcal{S}}$ \\
AIII & $\mathcal{S}_{-}$ & $U_{\mathcal{S}}$ & $i U_{\mathcal{C}} U_{\mathcal{S}}$ \\
\hline \hline
\end{tabular}

As explained in Ref. [21], the introduction of defect dimensions can be used to define the dimension-raising isomorphisms for tenfold-way classes with an order-two antiunitary (anti)symmetry, making use of the fact that the complex Shiozaki-Sato classes with antiunitary (anti)symmetry are isomorphic to real tenfold-way classes. Such an isomorphism is most easily constructed [21] by noticing that renaming the coordinates $\left(\boldsymbol{k}_{\perp}, \boldsymbol{\varphi}_{\|}\right) \rightarrow \tilde{\boldsymbol{k}}$ and $\left(\boldsymbol{\varphi}_{\perp}, \boldsymbol{k}_{\|}\right) \rightarrow \tilde{\boldsymbol{\varphi}}$ gives a Hamiltonian in the corresponding tenfold-way class; see the transformation law (B2). Such a transformation defines the isomorphism

$K\left(s \mid d, d_{\|}, D, D_{\|}\right)=K_{\mathrm{TF}}\left(s \mid d-d_{\|}+D_{\|}, D-D_{\|}+d\right)$.

Correspondingly, for the complex Shiozaki-Sato classes with an antiunitary symmetry the dimension-raising

TABLE XXI. The mapped representation of the unitary ordertwo (anti)symmetry $\mathcal{S}$ under application of the dimension-raising isomorphisms $\kappa_{\|}, \kappa_{\perp}, \rho_{\|}$, and $\rho_{\perp}$ for the real tenfold-way classes. The mapping of the Hamiltonian and the tenfold-way symmetries is given in Table XIX.

\begin{tabular}{lccc}
\hline \hline TF classes & $\mathcal{S}$ symmetry & $\kappa_{\|}, \rho_{\|}\left(U_{\mathcal{S}}\right)$ & $\kappa_{\perp}, \rho_{\perp}\left(U_{\mathcal{S}}\right)$ \\
\hline AI, AII, $D, C$ & $\mathcal{S}_{+}, \mathcal{S}_{-}$ & $\tau_{3} U_{\mathcal{S}}$ & $\tau_{0} U_{\mathcal{S}}$ \\
AI, AII, $D, C$ & $\mathcal{C} \mathcal{S}_{+}, \mathcal{C} \mathcal{S}_{-}$ & $\tau_{1} U_{\mathcal{S}}$ & $\tau_{2} U_{\mathcal{S}}$ \\
BDI, CII, DIII, CI & $\mathcal{S}_{++}, \mathcal{S}_{--}$ & $U_{\mathcal{C}} U_{\mathcal{S}}$ & $U_{\mathcal{S}}$ \\
BDI, CII, DIII, CI & $\mathcal{S}_{+-}, \mathcal{S}_{-+}$ & $U_{\mathcal{S}}$ & $U_{\mathcal{C}} U_{\mathcal{S}}$ \\
\hline \hline
\end{tabular}

isomorphisms are defined by first applying the above isomorphism to a real tenfold-way class, then using Teo and Kane's dimension-raising isomorphisms $\kappa$ and $\rho$ [52], and then using the inverse of the isomorphism (B7). From this procedure it is readily seen that for complex ShiozakiSato classes with an antiunitary order-two symmetry one has, up to the isomorphism (B7),

$$
\rho_{\|}=\kappa_{\perp}=\kappa, \quad \kappa_{\|}=\rho_{\perp}=\rho .
$$

Boundary homomorphism $\delta$.-From the definition (35) of the boundary map $\delta$, we can write the action of the homomorphism $\delta \circ \rho$

$$
\delta \circ \rho[H(\boldsymbol{k}, \boldsymbol{\varphi}, m)]=H_{\rho}(\boldsymbol{k}, \boldsymbol{\varphi}, m) .
$$

Together with the definition of $H_{\rho}$ given above this fully specified the product $\delta \circ \rho$.

\section{APPENDIX C: PROPERTIES OF THE ORDER-RAISING HOMOMORPHISM $\omega$}

The explicit expression (34) for the order-raising homomorphism $\omega$ arises naturally in the context of an exact sequence containing tenfold-way classifying groups $K_{\mathrm{TF}}$ and Shiozaki-Sato groups $K$. This exact sequence is a variant of an exact sequence considered by Turner et al. [6] and by us [16] for the classification of inversion-symmetric and mirror-symmetric topological insulators and superconductors,

$$
\begin{aligned}
K\left(d, d_{\|}, D, D_{\|}-1\right) & \stackrel{i}{\rightarrow} K_{\mathrm{TF}}(d, D) \\
& \stackrel{c_{\mathcal{S}}}{\rightarrow} K\left(d, d_{\|}, D, D_{\|}\right) \\
& \stackrel{\omega}{\rightarrow} K\left(d+1, d_{\|}+1, D, D_{\|}\right) \\
& \stackrel{i}{\rightarrow} K_{\mathrm{TF}}(d+1, D)
\end{aligned}
$$

Here, $i$ is the natural homomorphism, in the literature [22] also called a "symmetry forgetting functor," that identifies a member of Shiozaki-Sato group as a member of the corresponding tenfold-way group, and $c_{\mathcal{S}}$ is the homomorphism that constructs separable Hamiltonians

$$
c_{\mathcal{S}}[H]=\left(\begin{array}{cc}
H & 0 \\
0 & \mathcal{S} H
\end{array}\right)
$$

where the symmetry $\mathcal{S}$ has $d_{\|}$inverted spatial dimensions and $D_{\|}$inverted defect dimensions. The homomorphism $\omega$ is defined by Eq. (34), where-using the more general definitions of the maps $\rho_{\|}, \delta$, and $\kappa_{\|}$given in the previous Appendix - the homomorphism $\omega$ appearing here is a map between defect Hamiltonians.

We first show that exactness of the sequence (C1) leads to the three properties of the order-raising homomorphism 
listed in Sec. IV. Exactness of the sequence (C1) will then be shown at the end of this Appendix.

Properties 1-3 of the order-raising homomorphism.The maps in the exact sequence $(\mathrm{C} 1)$ all preserve the group operations (i.e., they are homomorphisms), and the image of every map is the same as the kernel of the subsequent one. Thus, exactness at $K_{\mathrm{TF}}(d, D)$ immediately gives that $\omega(H)$ is trivial if and only if $H$ is separable, i.e., $H \in c_{\mathcal{S}}[K]$. This proves the first property of the order-raising homomorphism $\omega$ listed in Sec. IV of the main text.

To prove the second property, we first notice that the natural homomorphism $i$ commutes with the dimensionraising isomorphisms, since the latter act the same way on the Hamiltonians from the tenfold-way and Shiozaki-Sato classes, see Sec. VI,

$$
i \circ \chi_{\|}=\chi \circ i, \quad i \circ \chi_{\perp}=\chi \circ i,
$$

with $\chi=\rho, \kappa$. Exactness of the sequence (C1) at $K\left(d, d_{\|}, D, D_{\|}\right)$and $K_{\mathrm{TF}}(d, D)$ yields the isomorphism

$$
\text { ker } \begin{aligned}
\omega & =\operatorname{img} c_{\mathcal{S}} \\
& =K_{\mathrm{TF}}(d, D) / K_{\mathrm{TF}, \mathcal{S}}\left(d, d_{\|}, D, D_{\|}-1\right),
\end{aligned}
$$

with $\operatorname{ker} \omega \subseteq K\left(d, d_{\|}, D, D_{\|}\right)$and $i[K]=K_{\mathrm{TF}, \mathcal{S}}=K / K^{\prime}$. Because of commutation relations (C3), we conclude that the dimension-raising isomorphisms preserve the subgroups $K_{\mathrm{TF}, \mathcal{S}}$, and from Eq. (C4) the same applies to the subgroups $\operatorname{ker} \omega$. Furthermore, the exactness at $K\left(d, d_{\|}, D, D_{\|}\right)$gives

$$
\operatorname{img} \omega=\operatorname{ker} i \text {, }
$$

thus the dimension-raising isomorphisms also preserve the subgroups img $\omega$. We conclude that the homomorphism $\omega$ commutes with the dimension-raising isomorphisms up to an automorphism of $\operatorname{img} \omega$. Since the groups img $\omega=K^{\prime}$ are at most $\mathbb{Z}$ and $\operatorname{Aut}(\mathbb{Z})=\mathbb{Z}_{2}$, the mentioned automorphism changes at most the sign of the topological invariants. Such sign change is inessential and therefore the dimension-raising isomorphisms preserve the bulk classifying groups of HOTPs $K^{(n)}$. This proves the second property of the order-raising homomorphism $\omega$.

We prove the third property using the explicit expression (34) for the $\omega$ homomorphism. First, by comparing the dimension of a nontrivial $\omega^{n}(H)$, where $H$ is a minimal canonical model $[9,21,78]$, a representative of $K / K^{\prime} \operatorname{ker} \omega$, to the minimal dimension of the representative of $K^{(n)} / K^{(n+1)}$ we find that $\omega^{n}(H)$ is also a minimal canonical model. We therefore conclude that for a minimal canonical model $H$, representative of either $K^{(n)} / K^{(n+1)}$ or $K / K^{\prime} \operatorname{ker} \omega, \omega(H)$ is also a minimal canonical model.
Next, we show that under the assumption that a minimal canonical model with $n-1$ crystalline-symmetry-breaking mass terms $H^{(n)}$ (for a fixed $n$ ) is a representative of $K^{(n-1)} / K^{(n)}$ for $n>1$ and $K / K^{\prime} \operatorname{ker} \omega$ for $n=1, \omega(H)$ has $n$ boundary mass terms. Since under these assumptions, $\omega(H)$ is a minimal canonical model, the number of its $\mathcal{S}$-symmetry-breaking mass terms does not change under the continuous Hamiltonian deformations. It is now a matter of simple algebra to show that there are no additional $\mathcal{S}$-symmetry-breaking mass terms beyond the ones given in Tables XV-XIV; we illustrate how this proof works for classes $\mathrm{BDI}^{\mathcal{S}_{++}}, \mathrm{BDI}^{\mathcal{S}_{--}}, \mathrm{CII}^{\mathcal{S}_{++}}$, and $\mathrm{CII}^{\mathcal{S}_{--}}$. Proofs for the other symmetry classes are analogous. In order to satisfy chiral symmetry, the additional mass term needs to be of the form $\tau_{3} M_{n+1}$ which has to anticommute with $M_{n}=\tau_{3} U_{\mathcal{C}}$. Thus, $M_{n+1}$ anticommutes with $U_{\mathcal{C}}$, which makes it a valid $\mathcal{S}$-symmetry-breaking mass term of the $H^{(n)}$ Hamiltonian, contradicting the initial assumption on the number of the crystalline symmetry breaking mass terms. This proves the first statement of the third property of the $\omega$ homomorphism. The second statement of the third property directly follows from exactneses of the sequence (C1) at $K(d+1$, $\left.d_{\|}+1, D, D_{\|}\right)$since ker $i$ consists of those Hamiltonians that have at least one crystalline-symmetry-breaking mass term.

Exactness of the sequence $(\mathrm{Cl})$.- - The exactness of the sequence $(\mathrm{C} 1)$ can be proven as follows: Consider a one-parameter family $H(\varphi)$ of a Hamiltonian $H$ from $K\left(d, d_{\|}, D, D_{\|}-1\right)$, with the order-two symmetry (antisymmetry) $U_{\mathcal{S}}$ acting locally as $\sigma_{\mathcal{S}} U_{\mathcal{S}} H(\varphi) U_{\mathcal{S}}^{\dagger}=H(\varphi)$. This one-parameter family is mapped via the homomorphism $c_{\mathcal{S}}$ i to $H^{\prime}$,

$$
H^{\prime}(\varphi)=H(\varphi) \oplus H(-\varphi),
$$

that is, the $\mathcal{S}$ symmetry now acts nonlocally on the coordinate $\varphi$. The loop (C6) is a topologically trivial loop. Alternatively, each topologically trivial loop can be deformed to the above form with an arbitrary $H(\varphi)$ proving that $\operatorname{img} i=\operatorname{ker} c_{\mathcal{S}}$.

We next show that every Hamiltonian in ker $\omega$ can be continuously deformed to the diagonal form (C2). Since $\kappa_{\|}$ and $\rho_{\|}$are isomorphisms that preserve a diagonal form, it is sufficient to show that every Hamiltonian in $\operatorname{ker} \delta$ can be deformed into the diagonal form. Here, to we note that $\delta(H)=0$ implies that $H(0)$ and $H(\pi)$ are both in the trivial equivalence class (nontrivial $H(0)=H(\pi)$ would correspond to a weak topological phase, which we do not consider here), for which after continuous deformation, we may set $H(0)=H(\pi)=e$, e being the trivial element. Under stable equivalence we may replace $H(\varphi)$ by $H(\varphi) \oplus$ $e$ which may be smoothly deformed into 
(a)

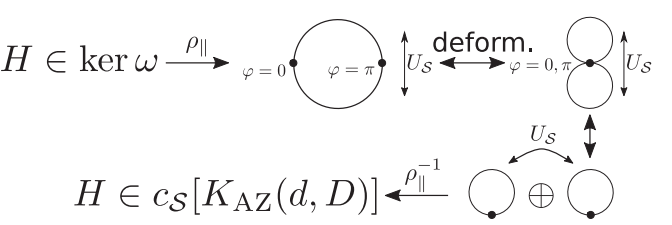

(b)

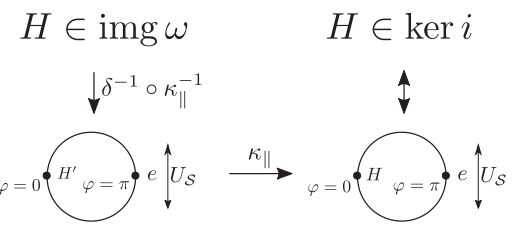

FIG. 11. Hamiltonians from ker $\omega$ can be deformed to the form (C2) and vice versa (a). For Hamiltonians from $\operatorname{img} \omega$, a path can be constructed that connects them to the trivial element (b). Similarly for every Hamiltonian in ker $i$, the loop in canonical form can be constructed that yields $H$ from $\operatorname{img} \omega$.

$$
H(\varphi) \equiv \begin{cases}H(\varphi) \oplus e & \text { for } 0 \leq \varphi<\pi \\ e \oplus H(\varphi) & \text { for } \pi \leq \varphi<2 \pi\end{cases}
$$

and subsequently, into a Hamiltonian of the form (C2), since $\rho_{\|} \mathcal{S} \rho_{\|}^{-1} H(2 \pi-\varphi)=H(\varphi)$. As the procedure can be run backwards we conclude $\operatorname{ker} \omega=\operatorname{img} c_{\mathcal{S}}$ giving the exactness of the sequence $(\mathrm{C} 1)$ at $K\left(d, d_{\|}, D, D_{\|}\right)$. Figure 11(a) illustrates the above steps of the proof.

Similarly, because $\kappa_{\|}$is an isomorphism, to show exactness at the second stage of the sequence $(\mathrm{C} 1)$ it is sufficient to show that any element of img $\delta$ can be smoothly deformed to the trivial element $e$ if the crystalline symmetry $\rho_{\|} \mathcal{S} \rho_{\|}^{-1}$ is no longer imposed, and vice versa; see Fig. 11(b). Again, we may assume that $H(0)=e$, and the continuous deformation linking $H(\pi) \ominus H(0)$ to $e \ominus e$ is $H(\varphi) \ominus H(0)$ with $0<\varphi<\pi$. Similarly, if such a transformation exists, i.e., if there exists a continuous function $\tilde{H}(\varphi)=$ $H(\varphi) \ominus H(0)$ interpolating between $H(0) \ominus H(0)$ and $H(\pi) \ominus H(0)$, then there also exists a family of $\rho_{\|} \mathcal{S} \rho_{\|}^{-1}$ symmetric Hamiltonians

$$
H(\varphi) \equiv \begin{cases}H(\varphi) & \text { for } 0 \leq \varphi<\pi \\ H(2 \pi-\varphi) & \text { for } \pi \leq \varphi<2 \pi\end{cases}
$$

such that $\tilde{H}(\varphi)=H(\varphi) \ominus H(0)$.

\section{APPENDIX D: CALCULATION OF ker $\omega^{k}$ AND $K_{\mathrm{TF}, \mathcal{S}}$}

Because the order-raising map $\omega$ commutes with the isomorphisms $\kappa_{\|}$and $\kappa_{\perp}$ it is sufficient to calculate the groups $\operatorname{ker} \omega$ and $K_{\mathrm{TF}, \mathcal{O}}$ for the case $d=d_{\|}=0$. The results for $d=0$ can be lifted to $d>0$ by suitable application of the dimension-raising isomorphisms $\kappa_{\|}$and $\kappa_{\perp}$. To obtain ker $\omega$ for $d=0$ we note that the bulkboundary correspondence (2) then gives

$$
K^{\prime}(1,1)=\mathcal{K}_{a}^{\prime \prime}=K(0,0) / \operatorname{ker} \omega,
$$

whereas $K_{\mathrm{TF}, \mathcal{S}}$ can be obtained from the isomorphism (C4). For most symmetry classes these two relations are sufficient to determine ker $\omega$ and $K_{\mathrm{TF}, \mathcal{S}}$ from the known groups [21] $K$ and [30,41] $K^{\prime}$, owing to the simple structure of these groups. There are a few cases for which both $K^{\prime}$ and ker $\omega$ are nontrivial and nonunique subgroups of $K$. For these an explicit calculation is needed. These special cases are considered below. The results for the groups ker $\omega$ and $K_{\mathrm{TF}, \mathcal{O}}$ for $d=0$ are given in Tables XXII-XXIV and XXV-XXVII, respectively.

$$
\begin{array}{r}
\text { 1. Classes } \mathrm{A}^{\mathcal{O}},(s, t)=(0,0), \mathrm{AI}^{\mathcal{O}_{+}},(s, t)=(\mathbf{0}, 0) \\
\text { and } \mathrm{AII}^{\mathcal{O}_{+}},(s, t)=(4,0)
\end{array}
$$

A zero-dimensional Hamiltonian $H_{0}$ in classes $\mathrm{A}$ and $\mathrm{AI}$ with an order-two on-site symmetry $\mathcal{O}$ is classified by

$$
K=\left\{\left(n_{+}, n_{-}\right), n_{+}, n_{-} \in \mathbb{Z}\right\}=\mathbb{Z}^{2},
$$

where $n_{ \pm}$is the difference between the number of positive and negative energy levels of $H_{0}$ with \pm parity under $\mathcal{O}$ symmetry. In class AII, due to Kramers degeneracy, the integers $n_{ \pm}$need to be even. Since the local symmetry $\mathcal{O}$ commutes with the time-reversal symmetry (class AI), the subgroups ker $i$ and ker $\omega$ are easily obtained,

$$
\begin{aligned}
\operatorname{ker} i & =\{(n,-n), n \in \mathbb{Z}\}=\mathbb{Z}, \\
\operatorname{ker} \omega & =\{(n, n), n \in \mathbb{Z}\}=\mathbb{Z},
\end{aligned}
$$

since Hamiltonians with $n_{+}=n_{-}=n$ can be deformed into a separable Hamiltonian, whereas Hamiltonians with $n_{+}+n_{-}=0$ are trivial when the protection by the on-site symmetry $\mathcal{O}$ is lifted.

2. Classes $\mathrm{BDI}^{\mathcal{O}_{++}},(s, t)=(\mathbf{1}, 0)$ and $\mathrm{D}^{\mathcal{O}_{+}},(s, t)=(\mathbf{2}, \mathbf{0})$

Hamiltonians $H$ from these classes are classified by

$$
K=\left\{\left(n_{+}, n_{-}\right), n_{ \pm} \in \mathbb{Z}_{2}\right\}=\mathbb{Z}_{2}^{2},
$$

with $n_{ \pm}=\operatorname{sign}\left[\operatorname{Pf}\left(H_{ \pm}\right)\right]$, where $H_{ \pm}$is the block of the Hamiltonian $H$ with \pm parity under $\mathcal{O}$. The Hamiltonian $H$

TABLE XXII. The subgroups $\operatorname{ker} \omega^{k} \subseteq K(s, t \mid 0,0,0,0)$ for complex Shiozaki-Sato classes with a unitary order-two (anti) symmetry.

\begin{tabular}{lccc}
\hline \hline$s$ & TF class & $t=0$ & $t=1$ \\
\hline 0 & A & $\mathbb{Z}$ & 0 \\
1 & AIII & 0 & 0 \\
\hline \hline
\end{tabular}


TABLE XXIII. The subgroups ker $\omega \subseteq K(s \mid 0,0,0,0)$ for complex Shiozaki-Sato classes with an antiunitary order-two (anti) symmetry. The integer in the superscript gives the $q$ so that $\operatorname{ker} \omega^{k}=K$ for $k>q$.

\begin{tabular}{lcc}
\hline \hline$s$ & Shiozaki-Sato class & ker $\omega$ \\
\hline 0 & $\mathrm{~A}^{\mathcal{T}^{+} \mathcal{O}}$ & $2 \mathbb{Z}^{(q=2)}$ \\
1 & $\mathrm{AIII}^{\mathcal{P}^{+} \mathcal{O}_{+}}$ & $0^{(q=1)}$ \\
2 & $\mathrm{~A}^{\mathcal{P}^{+} \mathcal{O}}$ & $\mathbb{Z}_{2}$ \\
3 & $\mathrm{AIII}^{\mathcal{T}^{-} \mathcal{O}_{-}}$ & 0 \\
4 & $\mathrm{~A}^{\mathcal{T}^{-} \mathcal{O}}$ & $2 \mathbb{Z}$ \\
5 & $\mathrm{AIII}^{\mathcal{P}^{-} \mathcal{O}_{+}}$ & 0 \\
6 & $\mathrm{~A}^{\mathcal{P}^{-} \mathcal{O}}$ & 0 \\
7 & $\mathrm{AIII}^{\mathcal{T}^{+} \mathcal{O}_{-}}$ & 0 \\
\hline \hline
\end{tabular}

TABLE XXIV. The subgroups ker $\omega \subseteq K(s, t \mid 0,0,0,0)$ for real Shiozaki-Sato classes with a unitary order-two (anti)symmetry. The integer in the superscript gives the integer $q$ so that $\operatorname{ker} \omega^{k}=K$ for $k>q$.

\begin{tabular}{lccccc}
\hline \hline$s$ & TF class & $t=0$ & $t=1$ & $t=2$ & $t=3$ \\
\hline 0 & AI & $\mathbb{Z}$ & 0 & $2 \mathbb{Z}$ & $\mathbb{Z}_{2}$ \\
1 & BDI & $\mathbb{Z}_{2}^{(q=1)}$ & 0 & 0 & $\mathbb{Z}_{2}$ \\
2 & D & $\mathbb{Z}_{2}^{(q=1)}$ & $0^{(q=1)}$ & 0 & 0 \\
3 & DIII & 0 & $0^{(q=1)}$ & 0 & 0 \\
4 & AII & $2 \mathbb{Z}$ & 0 & $4 \mathbb{Z}^{(q=2)}$ & 0 \\
5 & CII & 0 & 0 & 0 & 0 \\
6 & C & 0 & 0 & 0 & 0 \\
7 & CI & 0 & 0 & 0 & 0 \\
\hline \hline
\end{tabular}

TABLE XXV. The groups $K_{\mathrm{TF}}(s \mid 0,0)$ and $K_{\mathrm{TF}, \mathcal{O}}(s, t \mid 0,0,0,0)$ for complex Shiozaki-Sato classes with a unitary order-two (anti) symmetry.

\begin{tabular}{lcccc}
\hline \hline$s$ & TF class & $K_{\mathrm{TF}}(s)$ & $K_{\mathrm{TF}, \mathcal{O}}(s, 0)$ & $K_{\mathrm{TF}, \mathcal{O}}(s, 1)$ \\
\hline 0 & A & $\mathbb{Z}$ & $\mathbb{Z}$ & 0 \\
1 & AIII & 0 & 0 & 0 \\
\hline \hline
\end{tabular}

TABLE XXVI. The subgroups $K_{\mathrm{TF}, \mathcal{O}}(s \mid 0,0,0,0)$ for complex Shiozaki-Sato classes with an antiunitary order-two (anti)symmetry.

\begin{tabular}{lccc}
\hline \hline$s$ & Shiozaki-Sato class & $K_{\mathrm{TF}}(s)$ & $K_{\mathrm{TF}, \mathcal{O}}(s)$ \\
\hline 0 & $\mathrm{~A}^{\mathcal{T}^{+} \mathcal{O}}$ & $\mathbb{Z}$ & $\mathbb{Z}$ \\
1 & $\mathrm{AIII}^{\mathcal{P}^{+} \mathcal{O}_{+}}$ & 0 & 0 \\
2 & $\mathrm{~A}^{\mathcal{P}^{+} \mathcal{O}}$ & $\mathbb{Z}$ & 0 \\
3 & $\mathrm{AIII}^{\mathcal{T}^{-} \mathcal{O}_{-}}$ & 0 & 0 \\
4 & $\mathrm{~A}^{\mathcal{T}^{-} \mathcal{O}}$ & $\mathbb{Z}$ & $2 \mathbb{Z}$ \\
5 & $\mathrm{AIII}^{\mathcal{P}^{-} \mathcal{O}_{+}}$ & 0 & 0 \\
6 & $\mathrm{~A}^{\mathcal{P}^{-} \mathcal{O}}$ & $\mathbb{Z}$ & 0 \\
7 & $\mathrm{AIII}^{\mathcal{T}^{+} \mathcal{O}_{-}}$ & 0 & 0 \\
\hline \hline
\end{tabular}

TABLE XXVII. The groups $K_{\mathrm{TF}}(s \mid 0,0)$ and $K_{\mathrm{TF}, \mathcal{O}}(s, t \mid 0$, $0,0,0)$ for the real Shiozaki-Sato classes with a unitary ordertwo (anti)symmetry.

\begin{tabular}{lcccccc}
\hline \hline & & & \multicolumn{4}{c}{$K_{\mathrm{TF}, \mathcal{O}}(s, t)$} \\
$s$ & TF class & $K_{\mathrm{TF}}(s)$ & $t=0$ & $t=1$ & $t=2$ & $t=3$ \\
\hline 0 & AI & $\mathbb{Z}$ & $\mathbb{Z}$ & 0 & $2 \mathbb{Z}$ & 0 \\
1 & BDI & $\mathbb{Z}_{2}$ & $\mathbb{Z}_{2}$ & $\mathbb{Z}_{2}$ & 0 & 0 \\
2 & D & $\mathbb{Z}_{2}$ & $\mathbb{Z}_{2}$ & $\mathbb{Z}_{2}$ & $\mathbb{Z}_{2}$ & 0 \\
3 & DIII & 0 & 0 & 0 & 0 & 0 \\
4 & AII & $2 \mathbb{Z}$ & $2 \mathbb{Z}^{2}$ & 0 & $2 \mathbb{Z}$ & 0 \\
5 & CII & 0 & 0 & 0 & 0 & 0 \\
6 & C & 0 & 0 & 0 & 0 & 0 \\
7 & CI & 0 & 0 & 0 & 0 & 0 \\
\hline \hline
\end{tabular}

is taken in a basis where particle-hole antisymmetry is represented by $U_{\mathcal{P}}=1$. In this class, the subgroups ker $i$ and ker $\omega$ are identical,

$$
\begin{aligned}
\operatorname{ker} i & =\left\{(n, n), n \in \mathbb{Z}_{2}\right\}=\mathbb{Z}_{2}, \\
\operatorname{ker} \omega & =\left\{(n, n), n \in \mathbb{Z}_{2}\right\}=\mathbb{Z}_{2} .
\end{aligned}
$$

[1] M.Z. Hasan and C. L. Kane, Colloquium: Topological Insulators, Rev. Mod. Phys. 82, 3045 (2010).

[2] B. A. Bernevig and T. L. Hughes, Topological Insulators and Topological Superconductors (Princeton University Press, Princeton, New Jersey, 2013).

[3] X.-L. Qi and S.-C. Zhang, Topological Insulators and Superconductors, Rev. Mod. Phys. 83, 1057 (2011).

[4] L. Fu, Topological Crystalline Insulators, Phys. Rev. Lett. 106, 106802 (2011).

[5] C. Fang, M. J. Gilbert, and B. A. Bernevig, Bulk Topological Invariants in Noninteracting Point Group Symmetric Insulators, Phys. Rev. B 86, 115112 (2012).

[6] A. M. Turner, Y. Zhang, R. S. K. Mong, and A. Vishwanath, Quantized Response and Topology of Magnetic Insulators with Inversion Symmetry, Phys. Rev. B 85, 165120 (2012).

[7] C. Fang, M. J. Gilbert, and B. A. Bernevig, Entanglement Spectrum Classification of $C_{n}$-Invariant Noninteracting Topological Insulators in Two Dimensions, Phys. Rev. B 87, 035119 (2013).

[8] C.-K. Chiu, H. Yao, and S. Ryu, Classification of Topological Insulators and Superconductors in the Presence of Reflection Symmetry, Phys. Rev. B 88, 075142 (2013).

[9] T. Morimoto and A. Furusaki, Topological Classification with Additional Symmetries from Clifford Algebras, Phys. Rev. B 88, 125129 (2013).

[10] R.-J. Slager, A. Mesaros, V. Juricic, and J. Zaanen, The Space Group Classification of Topological Band Insulators, Nat. Phys. 9, 98 (2013).

[11] P. Jadaun, D. Xiao, Q. Niu, and S. K. Banerjee, Topological Classification of Crystalline Insulators with Space Group Symmetry, Phys. Rev. B 88, 085110 (2013). 
[12] X.-J. Liu, J. J. He, and K. T. Law, Demonstrating Lattice Symmetry Protection in Topological Crystalline Superconductors, Phys. Rev. B 90, 235141 (2014).

[13] A. Alexandradinata, C. Fang, M. J. Gilbert, and B. A. Bernevig, Spin-Orbit-Free Topological Insulators without Time-Reversal Symmetry, Phys. Rev. Lett. 113, 116403 (2014).

[14] X.-Y. Dong and C.-X. Liu, Classification of Topological Crystalline Insulators Based on Representation Theory, Phys. Rev. B 93, 045429 (2016).

[15] C.-K. Chiu, J. C. Y. Teo, A. P. Schnyder, and S. Ryu, Classification of Topological Quantum Matter with Symmetries, Rev. Mod. Phys. 88, 035005 (2016).

[16] L. Trifunovic and P. W. Brouwer, Bott Periodicity for the Topological Classification of Gapped States of Matter with Reflection Symmetry, Phys. Rev. B 96, 195109 (2017).

[17] J. Kruthoff, J. de Boer, J. van Wezel, C. L. Kane, and R.-J. Slager, Topological Classification of Crystalline Insulators through Band Structure Combinatorics, Phys. Rev. X 7, 041069 (2017).

[18] H. C. Po, A. Vishwanath, and H. Watanabe, SymmetryBased Indicators of Band Topology in the 230 Space Groups, Nat. Commun. 8, 50 (2017).

[19] B. Bradlyn, L. Elcoro, J. Cano, M. G. Vergniory, Z. Wang, C. Felser, M. I. Aroyo, and B. A. Bernevig, Topological Quantum Chemistry, Nature (London) 547, 298 (2017).

[20] E. Khalaf, H. C. Po, A. Vishwanath, and H. Watanabe, Symmetry Indicators and Anomalous Surface States of Topological Crystalline Insulators, Phys. Rev. X 8, 031070 (2018).

[21] K. Shiozaki and M. Sato, Topology of Crystalline Insulators and Superconductors, Phys. Rev. B 90, 165114 (2014).

[22] K. Shiozaki, M. Sato, and K. Gomi, Topology of Nonsymmorphic Crystalline Insulators and Superconductors, Phys. Rev. B 93, 195413 (2016).

[23] K. Shiozaki, M. Sato, and K. Gomi, Topological Crystalline Materials: General Formulation, Module Structure, and Wallpaper Groups, Phys. Rev. B 95, 235425 (2017).

[24] K. Shiozaki, M. Sato, and K. Gomi, Atiyah-Hirzebruch Spectral Sequence in Band Topology: General Formalism and Topological Invariants for 230 Space Groups, arXiv:1802.06694.

[25] R. Thorngren and D. V. Else, Gauging Spatial Symmetries and the Classification of Topological Crystalline Phases, Phys. Rev. X 8, 011040 (2018).

[26] J.-W. Rhim, J. H. Bardarson, and R.-J. Slager, Unified BulkBoundary Correspondence for Band Insulators, Phys. Rev. B 97, 115143 (2018).

[27] S. A. Parameswaran and Y. Wan, Viewpoint: Topological Insulators Turn a Corner, Physics 10, 132 (2017).

[28] F. Schindler, A. M. Cook, M. G. Vergniory, Z. Wang, S. S. P. Parkin, B. A. Bernevig, and T. Neupert, Higher-Order Topological Insulators, Sci. Adv. 4, eaat0346 (2018).

[29] Y. Peng, Y. Bao, and F. von Oppen, Boundary Green Functions of Topological Insulators and Superconductors, Phys. Rev. B 95, 235143 (2017).

[30] J. Langbehn, Y. Peng, L. Trifunovic, F. von Oppen, and P. W. Brouwer, Reflection-Symmetric Second-Order Topological Insulators and Superconductors, Phys. Rev. Lett. 119, 246401 (2017).
[31] Z. Song, Z. Fang, and C. Fang, (d-2)-Dimensional Edge States of Rotation Symmetry Protected Topological States, Phys. Rev. Lett. 119, 246402 (2017).

[32] W. A. Benalcazar, B. A. Bernevig, and T. L. Hughes, Quantized Electric Multipole Insulators, Science 357, 61 (2017).

[33] W. A. Benalcazar, B. A. Bernevig, and T. L. Hughes, Electric Multipole Moments, Topological Multipole Moment Pumping, and Chiral Hinge States in Crystalline Insulators, Phys. Rev. B 96, 245115 (2017).

[34] C. Fang and L. Fu, Rotation Anomaly and Topological Crystalline Insulators, arXiv:1709.01929.

[35] M. Ezawa, Higher-Order Topological Insulators and Semimetals on the Breathing Kagome and Pyrochlore Lattices, Phys. Rev. Lett. 120, 026801 (2018).

[36] H. Shapourian, Y. Wang, and S. Ryu, Topological Crystalline Superconductivity and Second-Order Topological Superconductivity in Nodal-Loop Materials, Phys. Rev. B 97, 094508 (2018).

[37] X. Zhu, Tunable Majorana Corner States in a Two-Dimensional Second-Order Topological Superconductor Induced by Magnetic Fields, Phys. Rev. B 97, 205134 (2018).

[38] Z. Yan, F. Song, and Z. Wang, Majorana Corner Modes in a High-Temperature Platform, Phys. Rev. Lett. 121, 096803 (2018).

[39] Y. Wang, M. Lin, and T. L. Hughes, Weak-Pairing Higher Order Topological Superconductors, Phys. Rev. B 98, 165144 (2018).

[40] Q. Wang, C.-C. Liu, Y.-M. Lu, and F. Zhang, HighTemperature Majorana Corner States, Phys. Rev. Lett. 121, 186801 (2018).

[41] M. Geier, L. Trifunovic, M. Hoskam, and P. W. Brouwer, Second-Order Topological Insulators and Superconductors with an Order-Two Crystalline Symmetry, Phys. Rev. B 97, 205135 (2018).

[42] G. van Miert and C. Ortix, Higher-Order Topological Insulators Protected by Inversion and Rotoinversion Symmetries, Phys. Rev. B 98, 081110 (2018).

[43] D. Calugaru, V. Juricic, and B. Roy, Higher Order Topological Phases: A General Principle of Construction, arXiv: 1808.08965.

[44] F. Schindler, Z. Wang, M. G. Vergniory, A. M. Cook, A. Murani, S. Sengupta, A. Y. Kasumov, R. Deblock, S. Jeon, I. Drozdov, H. Bouchiat, S. Guéron, A. Yazdani, B. A. Bernevig, and T. Neupert, Higher-Order Topology in Bismuth, Nat. Phys. 14, 918 (2018).

[45] G. E. Volovik, Topological Superfluid ${ }^{3} \mathrm{He}-\mathrm{B}$ in Magnetic Field and Ising Variable, JETP Lett. 91, 201 (2010).

[46] M. Sitte, A. Rosch, E. Altman, and L. Fritz, Topological Insulators in Magnetic Fields: Quantum Hall Effect and Edge Channels with a Nonquantized $\theta$ Term, Phys. Rev. Lett. 108, 126807 (2012).

[47] F. Zhang, C. L. Kane, and E. J. Mele, Surface State Magnetization and Chiral Edge States on Topological Insulators, Phys. Rev. Lett. 110, 046404 (2013).

[48] E. Khalaf, Higher-Order Topological Insulators and Superconductors Protected by Inversion Symmetry, Phys. Rev. B 97, 205136 (2018).

[49] A. Altland and M. R. Zirnbauer, Nonstandard Symmetry Classes in Mesoscopic Normal-Superconducting Hybrid Structures, Phys. Rev. B 55, 1142 (1997). 
[50] T. L. Hughes, E. Prodan, and B. A. Bernevig, InversionSymmetric Topological Insulators, Phys. Rev. B 83, 245132 (2011).

[51] A. Lau, J. van den Brink, and C. Ortix, Topological Mirror Insulators in One Dimension, Phys. Rev. B 94, 165164 (2016).

[52] J. C. Y. Teo and C. L. Kane, Topological Defects and Gapless Modes in Insulators and Superconductors, Phys. Rev. B 82, 115120 (2010).

[53] H. Isobe and L. Fu, Theory of Interacting Topological Crystalline Insulators, Phys. Rev. B 92, 081304 (2015).

[54] I. C. Fulga, N. Avraham, H. Beidenkopf, and A. Stern, Coupled-Layer Description of Topological Crystalline Insulators, Phys. Rev. B 94, 125405 (2016).

[55] S.-J. Huang, H. Song, Y.-P. Huang, and M. Hermele, Building Crystalline Topological Phases from LowerDimensional States, Phys. Rev. B 96, 205106 (2017).

[56] A. Matsugatani and H. Watanabe, Connecting HigherOrder Topological Insulators to Lower-Dimensional Topological Insulators, Phys. Rev. B 98, 205129 (2018).

[57] M. Nakahara, Geometry, Topology and Physics, 2nd ed., Graduate Student Series in Physics (CRC Press, Boca Raton, Florida, 2003).

[58] K. Shiozaki, M. Sato, and K. Gomi, $Z_{2}$ Topology in Nonsymmorphic Crystalline Insulators: Möbius Twist in Surface States, Phys. Rev. B 91, 155120 (2015).

[59] G. Kells, D. Meidan, and P. W. Brouwer, Low-Energy Subgap States in Multichannel p-Wave Superconducting Wires, Phys. Rev. B 85, 060507 (2012).

[60] S. Tewari and J. D. Sau, Topological Invariants for SpinOrbit Coupled Superconductor Nanowires, Phys. Rev. Lett. 109, 150408 (2012).

[61] V. Dwivedi, C. Hickey, T. Eschmann, and S. Trebst, Majorana Corner Modes in a Second-Order Kitaev Spin Liquid, Phys. Rev. B 98, 054432 (2018).

[62] In a nonminimal model (which can be obtained, e.g., by adding trivial bands to a minimal model of a topological phase), the number of mutually anticommuting $\mathcal{S}$-breaking mass terms $M_{l}$ may be less than $n-1$ or the $M_{l}$ do not all change sign under $\mathcal{S}$. However, in that case a Hamiltonian with $n-1$ mutually anticommuting $\mathcal{S}$-breaking mass terms may be recovered by removing or adding trivial bands.

[63] H. Song, S.-J. Huang, L. Fu, and M. Hermele, Topological Phases Protected by Point Group Symmetry, Phys. Rev. X 7, 011020 (2017).

[64] This property follows directly from the observation that the kernel of the natural quotient map $\tilde{\alpha}: K \rightarrow \alpha[K] / \alpha[G]$ is $G \operatorname{ker} \alpha$.
[65] T. I. Tuegel, V. Chua, and T. L. Hughes, Embedded Topological Insulators, arXiv:1802.06790.

[66] T. Zhang, Y. Jiang, Z. Song, H. Huang, Y. He, Z. Fang, H. Weng, and C. Fang, Catalogue of Topological Electronic Materials, arXiv:1807.08756.

[67] G. van Miert and C. Ortix, Dislocation Charges Reveal Two-Dimensional Topological Crystalline Invariants, Phys. Rev. B 97, 201111 (2018).

[68] A. Shitade, H. Watanabe, and Y. Yanase, Theory of Orbital Magnetic Quadrupole Moment and Magnetoelectric Susceptibility, Phys. Rev. B 98, 020407 (2018).

[69] Y. Gao, D. Vanderbilt, and D. Xiao, Microscopic Theory of Spin Toroidization in Periodic Crystals, Phys. Rev. B 97, 134423 (2018).

[70] Y. Gao and D. Xiao, Orbital Magnetic Quadrupole Moment and Nonlinear Anomalous Thermoelectric Transport, Phys. Rev. B 98, 060402 (2018).

[71] C. W. Peterson, W. A. Benalcazar, T. L. Hughes, and G. Bahl, A Quantized Microwave Quadrupole Insulator with Topologically Protected Corner States, Nature (London) 555, 346 (2018).

[72] M. Serra-Garcia, V. Peri, R. Süsstrunk, O. R. Bilal, T. Larsen, L. G. Villanueva, and S. D. Huber, Observation of a Phononic Quadrupole Topological Insulator, Nature (London) 555, 342 (2018).

[73] S. Imhof, C. Berger, F. Bayer, J. Brehm, L. W. Molenkamp, T. Kiessling, F. Schindler, C. H. Lee, M. Greiter, T. Neupert, and R. Thomale, Topolectrical-Circuit Realization of Topological Corner Modes, Nat. Phys. 14, 925 (2018).

[74] C. Yue, Y. Xu, Z. Song, Y.-M. Lu, H. Weng, C. Fang, and X. Dai, Symmetry Enforced Chiral Hinge States and Surface Quantum Anomalous Hall Effect in Magnetic Axion Insulator $\mathrm{Bi}_{2-x} \mathrm{Sm}_{x} \mathrm{Se}_{3}$, arXiv:1807.01414.

[75] Z. Wang, B. J. Wieder, J. Li, B. Yan, and B. A. Bernevig, Higher-Order Topology, Monopole Nodal Lines, and the Origin of Large Fermi Arcs in Transition Metal Dichalcogenides $X \mathrm{Te}_{2}(X=\mathrm{Mo}, W)$, arXiv:1806.11116.

[76] M. Ezawa, Strong and Weak Second-Order Topological Insulators with Hexagonal Symmetry and $\mathbb{z}_{3}$ index, Phys. Rev. B 97, 241402 (2018).

[77] In this work, we assume $\varphi$ to be defined on a torus rather than on a sphere around the defect, as in Refs. [21,52]. Defining $\varphi$ to be on a torus introduces weak invariants which are inessential for the present work, since we consider strong invariants only.

[78] C.-K. Chiu, Nontrivial Surface Topological Physics from Strong and Weak Topological Insulators and Superconductors, arXiv:1410.1117. 